\title{
Revestimentos Ramificados sobre Superfícies Compactas
}

\author{
Natalia Andrea Viana Bedoya
}

DISSERTAÇÃO APRESENTADA

$\mathrm{AO}$

INSTITUTO DE MATEMÁTICA E ESTATÍSTICA

DA

UNIVERSIDADE DE SÃO PAULO

PARA

OBTENÇÃO DO GRAU

$\mathrm{DE}$

MESTRE EM .IATEMÁTICA

Área de Toncentraçãu' "'mpologia Algébrica

Orientador: Prof. Dr. Daciberg Lima Gonçalves

Durante a elaboração deste trabalho, a autora recebeu apoio financeiro da CAPES e do CNPq. 


\title{
Revestimentos Ramificados sobre Superfícies Compactas
}

\author{
Este exemplar corresponde à redação final \\ da dissertação de mestrado devidamente \\ corrigida e defendida por \\ Natalia Andrea Viana Bedoya \\ e aprovada pela comissão julgadora.
}

São Paulo, março de 2004.

Banca examinadora:

- Prof. Dr. Daciberg Lima Gonçalves (Orientador) - IME-USP

- Profa. Dr. Lucilia Daruiz Borsari - IME-USP

- Prof. Dr. Derek Douglas Jack Hacon - PUC-RJ 


\section{Agradecimentos}

À minha mãe, meu tio e meu irmão por tudo.

Ao Prof. Daciberg Lima Gonçalves pela paciência, disponibilidade e boa vontade para me orientar.

A Profa. Lucilia Daruiz Borsari por estar sempre disposta a me ajudar nos momentos dificeis.

À Profa. Marta Macho Stadler pela motivação.

Aos meus amigos do IME, especialmente aos colombianos, e à Kika pela sua valiosa colaboração e amizade.

$\mathrm{E}$ a todos os que direta ou indiretamente contribuíram para a elaboração deste trabalho. 


\section{Resumo}

Um revestimento ramificado $\phi: M \longrightarrow N$ de grau $d \in \mathbb{Z}^{+}$, determina um conjunto de partições de $d$, o dado de ramificação. Neste trabalho estudamos os seguintes problemas de realizaçăo: dada uma superfície compacta e conexa $N, d \in \mathbb{Z}^{+}$e uma coleção de partições $\mathscr{D}$ de $d$ :

1. Existe um revestimento ramificado conexo de grau $d$ que realize $\mathscr{D}$ como dado de ramificação?

2. Dado $H \subset \pi_{1}(N)$ um subgrupo de índice finito $l$, existe um revestimento ramificado conexo que realize $\mathscr{D}$ e tal que $\phi_{*}\left(\pi_{1}(M)\right)=H$ ?

[EKS] responde a questão 1 quando $N$ é uma superfície fechada diferente de $\mathbb{S}^{2}$, e ambas questões quando $N=\mathbb{R} \mathrm{P}^{2}$ e $l=1$. [BGKZ1] (geometricamente) e [BGKZ2] (algebricamente) respondem a questão 2 para $N$ fechada diferente de $\mathbb{S}^{2}$ e de $\mathbb{R} \mathrm{P}^{2}$. No capítulo 6 deste trabalho respondemos as duas questões quando $N$ é uma superfície com bordo.

\footnotetext{
Abstract

A branched covering $\phi: M \longrightarrow N$ of degree $d$ determines a collection of partitions of $d$, the branch date. In this work we studied the following realization problems: given a compact connected surface $N, d \in \mathbb{Z}^{+}$and a collection $\mathscr{D}$ of partitions of $d$ : 1. Does there exist a connected branched coverig of degree $d$ realizing $\mathscr{D}$ like a branch date?

2. Given $H \subset \pi_{1}(N)$ a subgroup of finite index $l$, does there exist a connected branched covering realizing $\mathscr{D}$ and such that $\phi_{*}\left(\pi_{1}(M)\right)=H$ ?

[EKS] answers the first question when $N$ is a closed surface different of $\mathbb{S}^{2}$, and answers both questions when $N=\mathbb{R}^{2}$ and $l=1$. [BGKZ1] (by geometric arguments) and [BGKZ2] (by algebraic arguments) answer the second question when $N$ is a closed surface different of $\mathbb{S}^{2}$ and $\mathbb{R P}^{2}$. In chapter 6 of this work we answer the two questions when $N$ is a bordered surface.
} 


\section{Sumário}

1 Transformações Interiores 1

2 Revestimentos Ramificados 99

3 Sobre o Grupo Simétrico $\quad 21$

3.1 Resultados Básicos . . . . . . . . . . . . . . . . . 21

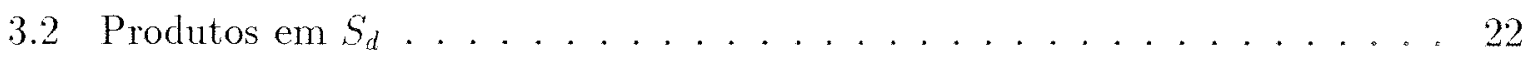

3.3 Construindo uma Operação de Colagem sobre Homomorfismos no Grupo Simétrico. . . . . . . . . . . . . . . . . . . . . 29

4 Revestimentos Ramificados sobre Superfícies Fechadas $\quad 37$

5 Revestimentos Ramificados Primitivos $\quad 51$

$\begin{array}{lll}6 & \text { Sobre Superfícies com bordo } & 58\end{array}$ 


\section{Introdução}

Todo revestimento ramificado $\phi: M \longrightarrow N$ de grau $d, d \in \mathbb{Z}^{+}$, sobre uma superfície compacta e conexa $N$ determina uma coleção de partições de $d$, o dado de ramificação.

O objetivo deste trabalho é apresentar resultados de realização de conjuntos de partições de um inteiro positivo $d$, como dado de ramificação de um revestimento ramificado conexo sobre uma superfície $N$ dada.

O estudo deste tipo de funções, iniciado por Hurwitz em 1891, foi motivado pelas propriedades locais das funções analíticas sobre superfícies de Riemann. No Capítulo 1 fazemos uma breve exposição da teoria das transformações interiores que nos permitirá trabalhar sobre qualquer tipo de superfícies. Usamos como referência os livros [AS] e [St].

No Capítulo 2, baseados nos artigos [EKS], [Ez] e [Hu], introduzimos a teoria dos revestimentos ramificados. Aqui, o principal resultado é o teorema de existência de Hurwitz.

O terceiro capítulo é dedicado a estudar propriedades do grupo simétrico, necessárias para avançar no problema. Para os resultados básicos usamos [DM], [Ro] e [Wi], e para o resto do capítulo [EKS], [E2], [BGKZ1] e [BGKZ2].

O Capítulo 4, baseado em [Hu], [EKS] e [Ez], apresenta resultados quando $N$ é fechada e diferente da esfera.

No quinto capítulo apresentamos resultados de realização mediante revestimentos ramificados primitivos, e uma generalização deste problema, sobre superfícies fechadas diferentes da esfera. Tais resultados encontram-se em [EKS] e [BGKZ2].

No Capítulo 6 , obtemos respostas quando $N$ é uma superfície com bordo. 


\section{Capítulo 1}

\section{Transformações Interiores}

O objetivo deste capítulo é introduzir a teoria básica das transformações interiores. Usamos como referencia os livros [AS] e [St].

No estudo das funções entre duas superfícies destaca-se a classe dos revestimentos ramificados. Foi Hurwitz quem, motivado pelas propriedades locais das funções analíticas e sobrejetoras entre duas superfícies de Riemann, iniciou este estudo. Seja $\phi: X \longrightarrow Y$ uma tal função. $\mathrm{O}$ fato de $\phi$ ter estas propriedades significa que para todo $y \in Y$ e para todo $x \in \phi^{-1}(y)$ existem vizinhanças $U_{y}, U_{x}$ respectivamente, $\phi\left(U_{x}\right) \subset U_{y}$, e homeomorfismos $\phi_{y}: U_{y} \longrightarrow A_{y}, \phi_{x}: U_{x} \longrightarrow A_{x}, A_{y}, A_{x}$ abertos do plano complexo centrados na origem com $\phi_{x}(x)=0=\phi_{y}(y)$, tais que a composição $h:=\phi_{y} \circ \phi \circ \phi_{x}^{-1}: A_{x} \longrightarrow A_{y}$ é analítica. Mais ainda, é possível tomar $\phi_{x}, \phi_{y}$ tais que para todo $z \in A_{x}, \operatorname{com} z \neq 0$, tem-se $h(z)=z^{k}{ }^{1}$

Como $h$ é analítica em $A_{x}$, para todo $z \in A_{x}, h(z)$ é da forma $\sum_{n=1}^{\infty} a_{n} z^{n}$. Assim, aquele $k$ é o primeiro índice tal que $a_{k} \neq 0$. Vamos chamar o inteiro $k$ índice de ramificação do ponto $x$, e se $k>1$ diz-se que $x$ é um ponto de ramificação.

Conhecemos as características da função $h$ :

1. A pré-imagem do zero é somente o zero.

2. Qualquer outro ponto tem $k$ pré-imagens.

\footnotetext{
${ }^{1}$ Ver [Fu], capítulo 19, seção 19. a, páginas 263-266.
} 
Portanto, $\phi: U_{x} \longrightarrow \phi\left(U_{x}\right)$ é do mesmo tipo. Sendo estas as propriedades inspiradoras para o desenvolvimento da teoria dos revestimentos ramificados e levando em consideração que são conseqüência de $\phi$ ser analítica e sobrejetora, surge a seguinte questão:

Se $f: S_{1} \longrightarrow S_{2}$ é uma função sobrejetora entre duas superfícies, que condições temos que impor sobre $f$ para que localmente se comporte como uma função analítica mesmo que alguma das superfícies seja não orientável?

Observa-se o seguinte:

Se $S_{1}, S_{2}$ são superfícies sobre as quais é possível definir uma estrutura complexa tal que elas se tornem superfícies de Riemann, basta pegar $f$ analítica. Mas acontece que todas as superfícies sobre as quais é possível definir tal estrutura são orientáveis. Aliás, $S$ é superfície de Riemann se e somente se $S$ é orientável. ${ }^{2}$

Para o caso das superfícies não orientáveis, foram estudadas as propriedades topológicas das funções analíticas que segundo [St], são conseqüências das duas seguintes:

1. Toda função analítica é aberta.

2. Nenhum conjunto contínuo ${ }^{3}$ é enviado por uma função analítica em um único ponto.

Define-se como Transformação interior toda transformação contínua entre duas superfícies que leve abertos em abertos e tal que nenhum conjunto continuo é levado em um ponto só, isto é, a pré-imagem de todo ponto é um conjunto totalmente desconexo.

Temos então que todo homeomorfismo é uma transformação interior assim como toda função analítica, e que a composição de transformações interiores é transformação interior.

Para o estudo das propriedades locais das transformações interiores entre superfícies, basta considerar a transformação entre planos euclidianos. Seja $f: E \rightarrow F$ uma transformação interior do plano $E$ no plano $F=f(E), q \in F$ e $p \in f^{-1}(q), D$ um domínio aberto $^{4}$ de $F \operatorname{com} q \in D$ e $\Delta=\bar{D}$. Denote por $(\Delta, p)$ o fecho da componente conexa por caminhos de $f^{-1}(D)$ que contém $p$. Vamos dizer que $(\Delta, p)$ é o domínio máximo de $\Delta$

\footnotetext{
2Ver [St], capítulo V, seção 1, página 102 .

${ }^{3}$ Conjunto fechado e conexo não unitário.

${ }^{4}$ Conjunto aberto e conexo.
} 
relacionado a $p$.

A imagem de $(\Delta, p)$ está contida em $\Delta$, mas pode não ocupar todo o domínio. Se $a \in \operatorname{Int}((\Delta, p))$ então $f(a) \in \operatorname{Int}(\Delta)$, e se $a \in \partial(\Delta, p)$ então $f(a) \in \partial(\Delta)$.

Define-se como domínio normal do plano $E$ por $f$, todo domínio $\delta \subset E$ fechado $^{5} \mathrm{e}$ compacto $^{6}$ tal que se $a \in \partial(\delta)$ então $f(a) \in \partial(f(\delta))$. $f(\delta)$ é um domínio fechado e limitado contido em $F$. Com a notação acima, tomando $\Delta=f(\delta)$ e $p \in \delta$ então $\delta=(\Delta, p)$. Reciprocamente, se $(\Delta, p)$, domínio máximo de um domínio $\Delta$ dado, é fechado e limitado em $E$, então ele é normal. De fato, a imagem de $(\Delta, p)$ é compacta e igual a $\Delta$.

Proposição 1.1 Se $f: E \longrightarrow F$ é uma transformação interior do plano no plano então são satisfeitas as seguintes propriedades:

(1) Se $\delta$ é um dominio normal, $\Delta=f(\delta), p \in \operatorname{Int}(\delta), \epsilon \Delta^{\prime} \subset \Delta$ é um domínio fechado $\operatorname{com} q=f(p) \in \Delta^{\prime}$, então o dominio $\left(\Delta^{\prime}, p\right)$ é normal.

(2) Dois domínios máximos quaisquer de $\Delta$ com um ponto em comum são iguaís.

(3) Dentro de uma vizinhança qualquer de $p$, existe um dominio normal contendo $p$.

Demonstração: (1) e (2) saem da definição de domínio máximo. Para (3), é suficiente pegar na vizinhança de $p$ uma curva fechada simples $\gamma$ em torno de $p$ que não passe por nenhum outro ponto $p^{\prime}$ tal que $f\left(p^{\prime}\right)=f(p)$. O conjunto dos pontos que satisfazem esta igualdade, segundo uma das condições das transformações interiores, é um conjunto totalmente desconexo. Seja então $\rho>0$ a distancia mínima de $f(\gamma)$ a $f(p)=q$. Se $\Delta$ é um domínio determinado por um círculo com centro em $q$ e raio $\rho$, o domínio $(\Delta, p)$ é normal, pois é interior a $\gamma$ e por isso é fechado e limitado.

Lema 1.2 Seja $f: E \longrightarrow F$ uma transformaçāo interior $e \delta$ um domínio normal de $E$ tal que $f(\delta)=\Delta$. A todo arco simples $\Sigma$ com ponto inicial $q$ e ponto final $l$ contido no interior de $\Delta$, e a todo ponto $p \in \delta$ tal que $f(p)=q$, corresponde um arco simples $\sigma$ com ponto inicial $p$ e contido no interior de $\delta$ tal que $f(\sigma)=\Sigma, e f: \sigma \longrightarrow \Sigma$ é um homeomorfismo.

\footnotetext{
${ }^{5}$ Conjunto fechado tal que seu interior é um domínio aberto e todos seus pontos de fronteira são pontos de acumulação de seus pontos interiores.

${ }^{6}$ Notemos que como neste caso estamos trabalhando sobre o plano, basta pedir que $\delta$ seja limitado.
} 
Demonstração: Seja $\Sigma:[0,1] \longrightarrow \Delta$, um caminho tal que $\Sigma(0)=q$ e $\Sigma(1)=l$. Encerremos cada um dos $2^{n}$ arcos determinados sobre $\Sigma$ por $t=\frac{i}{2^{n}}, t \in[0,1]$ e $i=$ $1, \ldots, 2^{n}$, com domínios fechados $\Delta_{n}^{i}$ tais que:

1. $\Delta_{n}^{i} \subset \Delta$.

2. $\Delta_{n}^{i} \cap \Delta_{n}^{j} \neq \emptyset$ se e somente se $j=i \pm 1$.

É fácil satisfazer estas duas condições cobrindo $\Sigma$ com círculos de centro em $\Sigma$ e raio $\rho$ fixo e suficientemente pequeno que dependa de $n$ tal que:

3. $\Delta_{n+1}^{i} \subset \Delta_{n}^{i^{\prime}}$, se o arco contido em $\Delta_{n+1}^{i}$ é um sub-arco do arco contido em $\Delta_{n}^{i^{\prime}}$.

4. Quando $n$ tenda a $\infty, \rho$ tenda a 0 .

O objetivo da última condição é que a união destes círculos tenda a $\Sigma$ quando $n$ tenda a infinito.

A cadeia $\left(\Delta_{n}^{i}\right), i=1, \ldots, 2^{n}$, faremos corresponder dentro de $\delta$ uma cadeia de domínios $\left(\delta_{n}^{i}\right)$ tal que para todo $i, \delta_{n}^{i}$ é o dominio máximo de $\Delta_{n}^{i}$.

Em primeiro lugar vamos construir $\left(\Delta_{n}^{1}, p\right)$ que será $\delta_{n}^{1}$. Já que ele está contido em $\delta$ é normal e sua imagem é todo $\Delta_{n}^{1}$. Existe portanto dentro de $\delta_{n}^{1}$, um ponto $p_{1}$ no mínimo tal que $f\left(p_{1}\right)=q_{1}$, onde $q_{i}$ é o ponto dado por $t=\frac{i}{2^{n}}$. O conjunto dos pontos $p_{1} \in \delta_{n}^{1}$ tais que $f\left(p_{1}\right)=q_{1}$ é fechado e compacto. Em torno de cada um destes pontos consideremos círculos $\sigma$ suficientemente pequenos tais que $f(\sigma)$ está contido no interior de $\Delta_{n}^{2}$, isto é possível pois $q_{1}$ está no interior deste domínio e $f$ é contínua. Pela compacidade, podemos escolher um número finito de $\sigma$ 's que cobrem o conjunto dos $p_{1}$ 's. Do item (2) da proposição 1.1 , dois domínios $\left(\Delta_{n}^{2}, p_{1}\right)$ e $\left(\Delta_{n}^{2}, p_{1}^{\prime}\right)$ com $p_{1}, p_{1}^{\prime}$ no mesmo $\sigma$ são iguais. Chamemos $\delta_{n}^{2}$ quaisquer dos $\left(\Delta_{n}^{2}, p_{1}\right)$. Sobre cada $\delta_{n}^{2}$ fazemos um procedimento análogo ao feito anteriormente sobre $\delta_{n}^{1}$ mas trocando $q_{1}$ por $q_{2}$ e obtemos assim um número finito de cadeias $\left(\delta_{n}^{i}\right)$. Define-se o número $n$ como o posto das cadeias $\left(\delta_{n}^{i}\right)$.

Seja $m$ um inteiro positivo. A cadeia $\left(\Delta_{n+m}^{i}\right), i=1, \ldots, 2^{n+m}$, construída no interior da cadeia $\left(\Delta_{n}^{i}\right), i=1, \ldots, 2^{n}$, é tal que os primeiros $2^{m}$ domínios estão contidos dentro do domínio $\Delta_{n}^{1}$, os $2^{m}$ domínios seguintes estão contidos no $\Delta_{n}^{2}$, e assim por diante. Cada $\Delta_{n}^{i}$ contém $2^{m}$ domínios da cadeia $\left(\Delta_{n+m}^{i}\right)$. Se $r<m$ é um inteiro positivo, a cadeia $\left(\Delta_{n+r}^{i}\right)$ está contida na cadeia $\left(\Delta_{n}^{i}\right)$ e contém a cadeia $\left(\Delta_{n+m}^{i}\right)$. Analogamente, uma 
cadeia $\left(\delta_{n+m}^{i}\right)$ está contida dentro de certa cadeia $\left(\delta_{n}^{i}\right)$, e se $r<m,\left(\delta_{n+r}^{i}\right)$ contém $\left(\delta_{n+m}^{i}\right)$ mas está contida na cadeia $\left(\delta_{n}^{i}\right)$. Entre as cadeias $\left(\delta_{1}^{i}\right)$, existe no mínimo uma que contém cadeias de todos os postos, pois o número de cadeias $\left(\delta_{1}^{i}\right)$ é finito. Peguemos uma cadeia de posto 1 que satisfaz esta condição. Entre as cadeias $\left(\delta_{2}^{i}\right)$ que ela contém, escolha uma que contém cadeias de todos os postos. Podemos continuar o processo indefinidamente e obtemos assim uma série de cadeias

$$
\left(\delta_{1}^{i}\right),\left(\delta_{2}^{i}\right), \ldots,\left(\delta_{n}^{i}\right), \ldots
$$

cada uma contida na anterior e contendo as seguintes. Assim a seqüência (1.1), é formada por conjuntos contínuos, limitados e encaixados. O conjunto comum $\sigma$ entre eles é um conjunto contínuo ou é um ponto só. Logo $\sigma$ deve conter no mínimo um ponto $x_{0}$ tal que $f\left(x_{0}\right)=y_{0}, \operatorname{com} y_{0} \in \Sigma$. De fato, todo conjunto de (1.1) contém um ponto que satisfaz esta condição. Pegando um destes pontos dentro de cada conjunto, qualquer ponto de acumulação do conjunto assim construído satisfaz a última igualdade e pertence a $\sigma$. Por outro lado, as cadeias $\left(\Delta_{n}^{i}\right)$ tendem a $\Sigma$ quando $n$ tende a infinito, donde $f(\sigma) \subset \Sigma \mathrm{e}$ portanto $f(\sigma)=\Sigma$. Resta mostrar que $f$ é injetora sobre $\sigma$, pois sendo contínua e $\sigma$ limitado esta restrição será um homeomorfismo. Sejam $a_{1}$ e $a_{2}$ dois pontos distintos de $\sigma$ tais que $f\left(a_{1}\right)=f\left(a_{2}\right)=b$. É claro que os domínios $\delta_{n}^{i}, \delta_{n}^{j}$ que contém $a_{1}, a_{2}$ são iguais ou são vizinhos, pois se os $\Delta_{n}^{i}, \Delta_{n}^{j}$ correspondentes têm interseç̧ão não vazia, eles são iguais ou $j=i \pm 1$. Eles formam então um conjunto contínuo $c_{n}$ e os conjuntos da série

$$
c_{1}, c_{2}, \ldots, c_{n}, \ldots
$$

são encaixados. Os $c_{n}$ tem um conjunto contínuo $c$ em comum que contém $a_{1}, a_{2}$. Portanto $f(c)=b$, já que bé o único ponto comum entre os $f\left(c_{n}\right)$, o que contradiz uma das condições das transformações interiores.

Lema 1.3 Dentro de todo domínio normal $\delta$ existe somente um número finito de caminhos $\sigma$ com ponto inicial $p$ satisfazendo as condiçöes do lema anterior.

Demonstração: Notemos que se $\sigma_{1}, \sigma_{2}$ são dois de tajs caminhos, o único ponto que têm em comum é $p$, seu ponto inicial. Por absurdo suponha que têm outro ponto em 
comum $s, s \neq p$. Então um sub-arco de $\sigma_{1}$ e um de $\sigma_{2}$ formam uma curva simples fechada determinando no plano um domínio de $\operatorname{Jordan}^{7} d$ limitado. $f(d)$ é um domínio fechado e limitado tendo por fronteira um subconjunto de $\Sigma$ o que é impossível, pois $\Sigma$ é simples.

Suponha agora que existem infinitos caminhos $\sigma_{1}, \sigma_{2}, \ldots$ que satisfazem as condições do lema anterior. Sejam $b_{1}, b_{2}, \ldots$ seus pontos finais respectivamente com $b_{i} \neq b_{j}$ se $i \neq j$. Já que os $b_{i}$ 's estão dentro de $\delta$ e $\delta$ é domínio normal, o conjunto de $b_{i}$ 's tem um ponto de acumulação $b_{0} \in \delta$ e é claro que $f\left(b_{0}\right)=l$. O ponto $b_{0}$ é interior a $\delta$ já que $\delta$ é normal e $l \in \operatorname{Int}(\Delta)$.

Prolonguemos $\Sigma$ por um arco $\Sigma^{\prime}$ com ponto inicial $l$ e ponto final $r \in \Delta$ tal que $\Sigma \Sigma^{\prime}$ seja um arco simples.

A cada um dos $b_{i}$ corresponde pelo lema anterior um caminho $\sigma_{i}^{\prime}$ tal que $f\left(\sigma_{i}^{\prime}\right)=\Sigma^{\prime}$ portanto $\sigma_{i} . \sigma_{i}^{\prime}$ se transforma em $\Sigma \Sigma^{\prime}$ por $f$, e os $\sigma_{i}^{\prime}$ não têm pontos em comum. Em particular, seus pontos finais $b_{i}^{\prime}$ são distintos e $f\left(b_{i}^{\prime}\right)=r$. Logo o conjunto dos $b_{i}^{\prime}$ tem um ponto de acumulação $b_{0}^{\prime} \in \delta$ tal que $f\left(b_{0}^{\prime}\right)=r$.

Em torno de $b_{0}$ e $b_{0}^{\prime}$ peguemos dois círculos com $b_{0}, b_{0}^{\prime}$ como centros e raio suficientemente pequeno tal que estejam contidos no interior de $\delta$. Existem dois caminhos, $\sigma_{j}^{\prime}, \sigma_{j+1}^{\prime}$, com seus pontos iniciais no círculo com centro em $b_{0}$ e com seus pontos finais no círculo com centro em $b_{0}^{\prime}$. Sejam $\alpha, \alpha^{\prime}$ caminhos entre estes círculos tais que $\alpha$ junta $b_{j} \operatorname{com} b_{j+1}$ e $\alpha^{\prime}$ junta $b_{j}^{\prime} \operatorname{com} b_{j+1}^{\prime}$. O caminho $\sigma_{j}^{\prime} \cdot \alpha^{\prime} \cdot \sigma_{j+1}^{\prime-1} \cdot \alpha^{-1}$ é uma curva fechada que determina um domínio de Jordan $d . f(d)$ tem todos os seus pontos fronteira sobre os caminhos $\Sigma, f\left(\alpha^{\prime}\right)$ e $f(\alpha)$, o que é impossível pois $f(d)$ é um domínio fechado e limitado.

Corolario 1.4 Se $q \in F, f^{-1}(q)$ é um conjunto de pontos isolados.

Demonstração: $f^{-1}(q)$ é fechado, pois $f$ é contínua. A prova da proposição reduz-se a mostrar que $f^{-1}(q)$ não tem pontos de acumulação no plano $E$.

Por absurdo, seja $p_{0}$ um ponto de acumulação de $f^{-1}(q)$. Pela proposição 1.1 é possível pegar um domínio normal $\delta$ tal que $p_{0} \in \delta$. Seja $\Delta=f(\delta), q=f\left(p_{0}\right) \in \operatorname{Int}(\Delta)$ e $\Sigma \subset \Delta$ um arco simples com ponto inicial $q$ e ponto final $q^{\prime}$. A cardinalidade de $f^{-1}(q)$ é infinita.

\footnotetext{
${ }^{7}$ Domínio compacto determinado por uma curva fechada simples no plano.
} 
Construímos caminhos $\sigma$ da forma que se fez na demonstração do lema 1.2 partindo dos pontos em $f^{-1}(q)$ e contidos em $\delta$. Pelo lema 1.3, cada um destes caminhos somente encontra um número finito dos outros. Seja $\sigma_{1}$ um caminho do conjunto, pela afirmação anterior existem infinitos caminhos que não cortam $\sigma_{1}$. Seja $\sigma_{2}$ um deles. Analogamente existe um número infinito de caminhos que não cortam nem $\sigma_{1}$ nem $\sigma_{2}$. Podemos continuar indefinidamente e obtemos assim uma série

$$
\sigma_{1}, \sigma_{2}, \ldots, \sigma_{n}, . .
$$

tal que $f\left(\sigma_{i}\right)=\Sigma$ e $\sigma_{i} \cap \sigma_{j}=\phi, i \neq j$, o que contradiz o lema 1.3.

Pelo corolário anterior, para todo $p \in E$ existe uma vizinhança $U_{p}$ de $p, U_{p} \subset E$ tal que se $x \in U_{p}$ e $x \neq p$ tem-se $f(x) \neq f(p)$.

Seja $\delta \subset U_{p}$ um domínio normal com $p \in \operatorname{Int}(\delta)$ e seja $\Gamma$ um círculo de centro $q=f(p)$, $\Gamma \subset \Delta=f(\delta)$ e $r \in C=\partial\left(\mathrm{I}^{\prime}\right)$. Existe um número finito de pontos $b_{i}, i=1, \ldots, m, b_{i} \in \delta$ tais que $f\left(b_{i}\right)=r$. Como $\delta$ é normal, pelo lema 1.2 existe um arco simples $\sigma_{1} \subset \delta$ partindo de $b_{1}$ tal que $f\left(\sigma_{1}\right)=C$, de tal modo que a todo ponto de $C$ diferente de $r$ corresponde um único ponto sobre $\sigma_{1}$ e a $r$ correspondem os extremos $b_{1}$ e $b_{2}$ de $\sigma_{1}$. Suponha $b_{1} \neq b_{2}$ e seja $\gamma_{2}$ um caminho análogo a $\gamma_{1}$ com ponto inicial $b_{2}$ e ponto final $b_{3}$. Deste modo obtemos uma curva fechada simples $\mathscr{C}$ interior a $\delta$. Seja $\gamma$ o domínio de Jordan determinado por $\mathscr{C}$ sobre $E . f(\gamma)$ é fechado e compacto e todos seus pontos fronteira estão sobre $C$. Logo $f(\gamma)$ coincide com $\Gamma$ já que $\gamma$ é normal e está contido em $\delta$. Quando percorremos $\mathscr{C} \mathrm{em}$ um sentido determinado, percorremos $n$ vezes $C, n \leq m$.

Se $n=1, f: \gamma \longrightarrow \Gamma$ é um homeomorfismo. De fato, suponha que no interior de $\gamma$ existem dois pontos $p_{1}, p_{2}$, tais que $f\left(p_{1}\right)=f\left(p_{2}\right)=l$. Considere um arco simples $\Sigma \subset \Gamma$ que passa pelos pontos $q, l$ e $r$. Existem dois arcos simples dentro de $\gamma$ partindo de $p_{1}, p_{2}$ que por $f$ viram sub-arcos de $\Sigma$ compreendidos entre $l$ e $r$, e dois arcos análogos partindo dos mesmos pontos que por $f$ viram sub-arcos de $\Sigma$ compreendidos entre $q$ e $l$. Os primeiros têm como ponto final $b$, único ponto de $\gamma$ tendo $r$ como imagem, pois $n=1$. Os últimos têm como ponto final $p$, único ponto de $U_{p}$ com $q$ como imagem. Existe então um domínio de Jordan $d$ determinado por estes arcos ou por alguns de seus sub-arcos. $f(d)$ é fechado e compacto e toda sua fronteira está sobre $\Sigma$, mas $\Sigma$ é um arco 
simples e portanto $p_{1}=p_{2}$. Por outro lado, a transformação sobre $\mathscr{C}$ mostra que todo ponto de $C$ inclusive $r$ tem somente uma pré-imagem. Logo, neste caso, $f: \gamma \longrightarrow \Gamma$ é um homeomorfismo.

Suponha $n>1$. Sejam $b_{1} \neq b_{2}$ e $\sigma_{1}, \sigma_{2}$ dois arcos simples partindo de $b_{1}, b_{2}$ respectivamente que viram $\Sigma$ por $f$. Estes dois arcos são interiores a $\gamma\left(\right.$ exceto $\left.b_{1}, b_{2}\right)$, pois $p \in \operatorname{Int}(\delta)$ e nenhum deles corta $\mathscr{C}$. p é o único ponto comum entre eles. De fato, suponha $t$ outro ponto comum, o primeiro que achamos ao percorrer $\sigma_{1}, \sigma_{2}$ de $b_{1}, b_{2}$ a $p$. Os sub-arcos determinados por $b_{1} t, b_{2} t$ e $b_{1} b_{2}$ determinam um domínio de Jordan $d \subset \gamma \mathrm{e}$ $p \notin d$, assim nenhum ponto dele tem $q$ como imagem. Mas $f(d)$ é fechado e compacto e toda sua fronteira está sobre $\Sigma$ e $C, \log o f(d)=\Gamma$, e temos uma contradição pois $q \in \Gamma$ não tem pré-imagem.

Sejam $b_{1}, b_{2}$ consecutivos sobre $\mathscr{C}$. O domínio de Jordan determinado por $\sigma_{1}, \sigma_{2}$ e o arco determinado por $b_{1} b_{2}$ transforma-se em $\Gamma$ por $f$. A transformação é um homeomorfismo sobre cada $\sigma_{i}$ e sobre o arco $b_{1} b_{2}$. Todo ponto interior se transforma em pontos de $\Gamma$ que não estão em $\Sigma$ nem em $C$, e a transformação no interior também é homeomorfismo. Temos demonstrado o seguinte teorema:

Teorema 1.5 Seja $f: S_{1} \longrightarrow S_{2}$ uma transformação interior entre superficies, $p \in S_{1}$. Então dentro de toda vizinhança de $p$, existe um dominio de Jordan $\gamma$ normal, tendo $p$ como ponto interior tal que é possivel dividir $\gamma \mathrm{em} n$ setores por meio de $n$ arcos simples que têm seus pontos finais na fronteira de $\gamma$ e sendo $p$ o ponto inicial de todos $e$ o único ponto comum. Além disso, f restrita ao interior de cada setor é um homeomorfismo assim como sobre cada um dos arcos de divisão de $\gamma$.

Em resumo, temos que para todo $p \in S_{1}$ e $q=f(p) \in S_{2}$ existem vizinhanças $U_{p}, U_{q}$ sobre as quais a função $f: U_{p} \longrightarrow U_{q}$ é tal que a única pré-imagem de $q$ é $p$ e qualquer outro ponto tem $n$ pré-imagens. Assim, quando $n=1$, sobre $U_{p}, f$ é homeomorfismo local, mas se $n>1$, dizemos que $p$ é um ponto de ramificação. 


\section{Capítulo 2}

\section{Revestimentos Ramificados}

Neste capítulo introduzimos a teoria dos revestimentos ramificados baseados nos artigos $[\mathrm{Hu}],[\mathrm{EKS}]$ e $[\mathrm{Ez}]$.

Seja $N^{-}=N \cup B$ uma superfície, onde $N$ é uma superfície sem bordo e as componentes conexas de $B$ são homeomorfas a $\mathbb{S}^{1}$ ou a $\mathbb{R}$. Caso $B=\emptyset, N^{-}$é simplesmente uma superfície sem bordo. ${ }^{1}$ No que segue, vamos supor $N^{-}$conexa e localmente conexa por caminhos (portanto, conexa por caminhos).

Um revestimento ramificado sobre $N^{-}$, é um conjunto $\left(M^{-}, \phi, N^{-}, B_{\phi}\right)$ tal que:

1. $M^{-}$é uma superfície não necessariamente conexa, que chamaremos superfície de revestimento.

2. $\phi: M^{-} \longrightarrow N^{-}$é uma função contínua, sobrejetora e interior.

3. $B_{\phi}$ é um conjunto discreto contido em $N^{-}$tal que $B_{\phi} \cap B=\emptyset$ e é a imagem dos pontos de ramificação de $M^{-}$por $\phi .^{2}$ Isto é, $\phi: \phi^{-1}\left(N-B_{\phi}\right) \longrightarrow\left(N-B_{\phi}\right)$ é um revestimento näo ramificado de grau $d$.

Chamaremos à restrição $\phi^{-1}\left(N^{-}-B_{\phi}\right) \longrightarrow N^{-}-B_{\phi}$ o revestimento não ramificado associado a $\left(M^{-}, \phi, N^{-}, B_{\phi}\right)$.

\footnotetext{
${ }^{1}$ Ver [AS], capítulo I, seção 13 página 23.

${ }^{2} \mathrm{Na}$ literatura faz-se um abuso de linguagem e diz-se que o conjunto $B_{\phi}$ é o conjunto dos pontos de ramificação.
} 
Se a superfície de revestimento $M$ é conexa, dizemos que $\left(M, \phi, N, B_{\phi}\right)$ é um revestimento ramificado conexo.

Que $\phi: \phi^{-1}\left(N-B_{\phi}\right) \longrightarrow\left(N-B_{\phi}\right)$ seja um revestimento nâa ramificado de grau $d$ quer dizer que para todo $x_{0} \in N^{-}-B_{\phi}$ existe uma vizinhança aberta $U_{x_{o}}$ tal que as componentes conexas de $\phi^{-1}\left(U_{x_{0}}\right)$ são homeomorfas a $U_{x_{0}}$ por $\phi$, e que a cardinalidade do conjunto $\phi^{-1}\left(x_{0}\right), \#\left(\phi^{-1}\left(x_{0}\right)\right)$, é igual a $d$.

Já que $B_{\phi}=\left\{x_{i}\right\}_{i \in I}$ é discreto, para todo $x_{i} \in B_{\phi}$ existe uma vizinhança aberta $V_{x_{i}}$ tal que $V_{x_{i}} \cap B_{\phi}=\left\{x_{i}\right\}$ e $\phi$ restrita a cada uma das componentes conexas $\tilde{V}_{i}$ de $\phi^{-1}\left(V_{x_{i}}\right)$, é do tipo $z \longmapsto z^{a_{i}}$, sendo $a_{i_{j}} \in \mathbb{Z}^{+}$o índice de ramificação local, para $j \in J_{i}$ com $\#\left(J_{i}\right)=\#\left(\phi^{-1}\left(x_{i}\right)\right)$. Isto é, na restrição $\phi: \tilde{V}_{i_{j}} \longrightarrow V_{x_{i}}$ qualquer $x \neq x_{i}$ tem $a_{i_{j}}$ pré-imagens sendo que $x_{i}$ só tem uma. É justamente este o motivo pelo qual tiramos $B_{\phi}$ na condição 3 . Além disso, a mesma condição implica que $\sum_{j \in J_{i}} a_{i_{j}}=d$, assim cada um $\operatorname{dos} x_{i} \in B_{\phi}$, determina uma partição $A_{i}=\left[a_{i_{j}}\right]_{j \in J_{i}}$ de $d$ dada pelos índices locais de ramificação. Denotemos por $\mathscr{D}$ o conjunto destas partições, $\mathscr{D}=\left\{A_{i}\right\}_{i \in l}$, e vamos chamá-lo dado de ramificação.

Para cada $x_{i} \in B_{\phi}$ definimos:

$$
\nu\left(A_{i}\right)=\sum_{j \in J_{i}}\left(a_{i}-1\right)
$$

como o defeito do ponto $x_{i}$. Este defeito dá conta da quantidade de pontos que fazem falta na pré-imagem de $x_{i}$ para ser um ponto "bom", no sentido de não ser imagem de um ponto de ramificação. Portanto $x \notin B_{\phi}$ implica $\nu(A)=0$, pois $A=[1,1, \ldots]$.

Definimos o defeito do revestimento como:

$$
\nu(\mathscr{D})=\sum_{i \in I} \nu\left(A_{i}\right)
$$

É muito interessante entender o que acontece quando $B \neq \emptyset$, lembremos que $B \cap B_{\phi}=\emptyset$.

Seja $\{B\}$ o conjunto das componentes conexas de $B$. Se $C \in\{B\}, C$ é homeomorfa a $\mathbb{S}^{1}$ ou a $\mathbb{R}$ e $\phi: \phi^{-1}(C) \longrightarrow C$ é um revestimento não ramificado.

Se $C$ é uma linha, $\phi^{-1}(C)$ tem $d$ componentes conexas e todas são linhas. Neste caso vamos definir o defeito de $C$ igual a zero, $\nu(C)=0$. 
Se $C$ é homeomorfa a $\mathbb{S}^{1}$, seja $K$ o conjunto de componentes conexas de $\phi^{-1}(C)$. Se $C_{t} \in K, C_{t}$ é um círculo ou uma linha. Se $C_{t}$ é uma linha então $\phi: C_{t} \longrightarrow C$ é o revestimento universal de $\mathbb{S}^{1}$ que tem um número infinito $a_{t}$ de folhas, $a_{t}=\infty$. Mas se $C_{t}$ é homeomorfo a $\mathbb{S}^{1}$, então $\phi: C_{t} \longrightarrow C$ é um revestimento com um número $a_{t}$ finito de folhas. Neste caso definimos o defeito de $C \operatorname{como} \nu(C)=\Sigma_{t \in T}\left(a_{t}-1\right)$, \#(T)=\#(K).

Vamos dizer que o revestimento $\left(M^{-}, \phi, N^{-}, B_{\phi}\right)$ é năo ramificado em $C$ se $\nu(C)=$ $0^{3} \quad$ Note que isto só acontece quando todas as componentes conexas de $\phi^{-1}(C)$ são homeomorfas a $\mathbb{S}^{1}$ e para cada uma delas o revestimento $\phi: C_{t} \longrightarrow C$ é a identidade, isto é, quando o número de componentes conexas é igual ao grau do revestimento.

Da discussão anterior é claro que as componentes conexas do bordo também determinam uma partição do grau do revestimento cujos termos podem ser finitos ou infinitos dependendo do caso.

Se $\mathscr{D}$ é um conjunto de partições não triviais de um inteiro positivo $d$, vamos dizer que $\mathscr{D}$ é realizável se existe um revestimento ramificado conexo tendo $\mathscr{D}$ como dado de ramificação.

Surge a seguinte questão geométrica:

Questão 1 Dada uma superficie $N^{-}=N \cup B$, um candidato d para grau e um conjunto de partiçôes $\mathscr{D}=\left\{A_{i}\right\}_{i \in I}$ de d onde cada termo de $A_{i}, i \in I$, é finito, será que existe um revestimento ramificado conexo $\left(M^{-}, \phi, N^{-}, B_{\phi}\right)$ tal que realize $\mathscr{D}$ ?

A resposta foi dada por Hurwitz e em essência o que ele fez foi traduzir o problema geométrico para um problema algébrico que consiste na realização de um determinado subgrupo de permutações do grupo simétrico $\operatorname{Sym}(\Omega)$ de um conjunto $\Omega$ com $d$ elementos.

Antes de enunciar e demonstrar o teorema vamos estudar um pouco algumas implicações da existência destes revestimentos.

Seja $\left(M^{-}, \phi, N^{-}, B_{\phi}\right)$ um revestimento ramificado de grau $d, x_{i} \in B_{\phi}$ e $V_{x_{i}}$ uma vizinhança de $x_{i}$ tal que $\phi$ restrita às componentes conexas $\widetilde{V}_{i_{j}}$ de $\phi^{-1}\left(V_{s_{i}}\right)$ é do tipo $z \longmapsto z^{a_{i_{j}}}$,

\footnotetext{
${ }^{3}$ Observemos que aqui o termo ramificação não tem nada a ver com pontos de ramificação, simplesmente descreve o caso em que o número de componentes conexas de $\phi^{-1}(C)$ é diferente de $d$.
} 
$j \in J_{i}$. Seja $\phi^{-1}\left(x_{i}\right)=\left\{y_{j}\right\}_{j \in J_{i}}, V_{x_{i}}^{\circ}=V_{x_{i}}-\left\{x_{i}\right\}$ e $\widetilde{V}_{i_{j}}^{\circ}=\widetilde{V}_{i,}-\left\{y_{j}\right\}$. $\hat{\mathrm{E}}$ claro que $\phi: \bigcup_{j \in J_{i}} \tilde{V}_{i_{j}} \longrightarrow V_{x_{i}}$ é um revestimento ramificado tendo a ramificação localizada em $x_{i}$, e $\phi: \bigcup_{j \in J_{i}} \tilde{V}_{i_{j}}^{\circ} \longrightarrow V_{x_{i}}^{\circ}$ é seu revestimento não ramificado associado. Pela teoria geral de revestimentos ${ }^{4}$ sabemos que existe uma ação a direita do grupo $\pi_{1}\left(V_{x_{i}}^{\circ}, x_{0_{i}}\right)$, sobre o conjunto $\Omega_{i}=\phi^{-1}\left(x_{0_{i}}\right)=\left\{z_{l}\right\}_{l=1}^{d}$, definida por

$$
\begin{aligned}
\Omega_{i} \times \pi_{1}\left(V_{x_{i}}^{\circ}, x_{0_{i}}\right) & \longrightarrow \Omega_{i} \\
\left(z_{l}, \alpha\right) & \longmapsto z_{l}, \alpha=\widetilde{\alpha}(1)
\end{aligned}
$$

onde $\widetilde{\alpha}(1)$ é o ponto final do único levantamento de um representante da classe $\alpha$ tendo $z_{l}$ como ponto inicial. Além disso, a ação será transitiva se e somente se $\bigcup_{j \in J_{i}} \widetilde{V}_{i j}^{o}$ é conexo. No nosso caso $\pi_{1}\left(V_{x_{i}}^{\circ}, x_{0_{i}}\right) \cong \mathbb{Z}$, seja $\alpha_{x_{i}}$ seu gerador. Logo é $\alpha_{x_{i}}$ quem determina (2.1). Para todo $i \in I$ e $j \in J_{i}, \phi: \widetilde{V}_{i j}^{\circ} \longrightarrow V_{x_{i}}^{\circ}$ é um revestimento não ramificado conexo, portanto $\pi_{1}\left(V_{x_{i}}^{\circ}, x_{0_{i}}\right)$ age transitivamente sobre $\Omega_{i_{j}}=\Omega_{i} \cap \widetilde{V}_{i_{j}}^{o}$ e mais que isso, a ação é simplesmente uma permutação cíclica dos elementos de $\Omega_{i_{j}}$, isto é, podemos considerar $\Omega_{i_{j}}=\left\{z_{j_{1}}, z_{j_{2}}, \ldots, z_{j_{a_{i}}}\right\}$ tal que $z_{j_{s}} . \alpha_{x_{i}}=z_{j_{s+1}}, s=1, \ldots, a_{i_{j}}-1$ e $z_{j_{a_{i}}} . \alpha_{x_{i}}=z_{j_{1}}$. Assim, o homomorfismo associado ${ }^{5}$ a esta ação é:

$$
\begin{aligned}
\Psi_{i}: \pi_{1}\left(V_{x_{i}}^{\circ}, x_{0_{i}}\right) & \longrightarrow \operatorname{Sym}\left(\Omega_{i}\right) \\
\alpha_{x_{i}} & \longmapsto \prod_{j \in J_{i}}\left(z_{j_{1}} z_{j_{2}} \ldots z_{j_{\alpha_{j}}}\right)
\end{aligned}
$$

donde a ação de cada $\alpha_{x_{i}}$ sobre $\Omega_{i}$ descreve completamente a ramificação sobre $x_{i}$.

Seja $\widehat{N}=N^{-}-B_{\phi}$ e $z^{*} \in \widehat{N}$. Já que $\widehat{N}$ é conexo por caminhos, existe um caminho $v_{i}$ de $z^{*}$ a $x_{0_{i}}$. Notemos que a classe $\left\{v_{i}^{*}\right\}$ do caminho $v_{i}^{*}=v_{i} \cdot \alpha_{x_{i}} \cdot v_{i}^{-1}$ age sobre $\Omega=\phi^{-1}\left(z^{*}\right) \mathrm{e}$ descreve completamente a mesma ramificação determinada por $\alpha_{x_{i}}$. Uma situação análoga temos para cada $C^{q} \in\{B\}$ homeomorfa a $\mathbb{S}^{1}$. Notemos que $\pi_{1}\left(C^{q}, \hat{x_{q}}\right)$ é cíclico infinito, $\operatorname{com}\left\{C^{q}\right\}$ como gerador dado por uma parametrização apropriada, e sua ação sobre $\phi^{-1}\left(\hat{x_{q}}\right)$ determina completamente a ramificação sobre $C^{q}$. Seja $w_{q}$ um caminho em $\widehat{N}$ tal que $w_{q}(0)=z^{*}, w_{q}(1)=\hat{x_{q}}$ e para todo $t \in(0,1) w_{q}(t) \in N$. A ação sobre $\Omega$ da classe $\left\{w_{q}^{*}\right\}$ do caminho $w_{q}^{*}=w_{q} \cdot\left\{C^{q}\right\} \cdot w_{q}^{-1}$ descreve a mesma ramificação determinada por $\left\{C^{9}\right\}$.

\footnotetext{
${ }^{4}$ Ver [Ma], capítulo $V$, seção 7 , página 161.

${ }^{5}$ Ver [DM] página 5.
} 
Logo o homomorfismo que corresponde à ação descrita acima é

$$
\begin{aligned}
\Psi: \pi_{1}\left(\widehat{N}, z^{*}\right) & \longrightarrow \operatorname{Sym}(\Omega) \\
\left\{v_{i}^{*}\right\} & \longmapsto \sigma_{x_{i}} \\
\left\{w_{q}^{*}\right\} & \longmapsto \lambda_{q}
\end{aligned}
$$

onde o conjunto dos comprimentos dos ciclos dados pela decomposição única das permutações $\sigma_{x_{i}}$ e $\lambda_{q}$ descrevem a ramificação sobre $x_{i}$ e $C^{q}$ respectivamente. É importante notar que para garantir a conexidade de $M^{-}$basta a existencia de uma classe $\beta \in \pi_{1}\left(\widehat{N}, z^{*}\right)$ tal que a permutação $\Psi(\beta)$ seja um ciclo de comprimento $d$, pois isto dá conta da transitividade da ação. Chamaremos o homomorfismo $\Psi$ a representação de Hurwitz associada ao revestimento.

Considerando a notação acima enunciamos algebricamente a Questão 1:

Seja $N^{-}$uma superficie, $d \in \mathbb{Z}^{+}$e $\mathscr{D}=\left\{A_{i}\right\}_{i \in I}$ um conjunto de partiçós de d onde cada termo de $A_{i}$ é finito. Seja $\widehat{N}=N^{-}-\left\{x_{i}\right\}_{i \in I}$ e $\Omega$ um conjunto com d elementos. Será que existe um homomorfismo $\Psi: \pi_{1}\left(\widehat{N}, z^{*}\right) \rightarrow \operatorname{Sym}(\Omega)$ tal que para todo $i \in I$ o conjunto dos comprimentos dos ciclos da permutação $\Psi\left(\left\{v_{i}^{*}\right\}\right)$ determine a partição $A_{i}$, $e$ que a açäo associada a $\Psi$ de $\pi_{1}\left(\widehat{N}, z^{*}\right)$ sobre $\Omega$ seja transitiva?

Teorema 2.1 (Hurwitz) Seja $N^{-}=N \cup B$ uma superficie, $\mathscr{D}=\left\{A_{i}\right\}_{i \in I}$ uma coleçäo de partiçoes de um inteiro positivo d (não necessariamente finito) onde cada termo da partição $A_{i}, i \in I$, é finito. $\mathscr{B}=\left\{B_{q}\right\}_{q \in Q}$ uma coleção de partiçoes de d, onde $\#(Q)=$ $\#(\{B\})$ e os termos das partições $B_{q}$ podem ser finitos ou infinitos. Então existe um revestimento ramificado de grau d, $\left(M^{-}, \phi, N^{-}, B_{\phi}\right)$, tendo $\mathscr{D}$ como dado de ramificaşâo se e somente se existe uma ação do grupo $\pi_{1}\left(\widehat{N}, z^{*}\right)$ sobre um conjunto $\Omega$ com delementos tal que as órbitas dadas pela ação das classes $\left\{v_{i}^{*}\right\}$ e de $\left\{w_{q}^{*}\right\}$ sobre $\Omega$ determinam as partiçoes $A_{i}, B_{q}$ respectivamente. Mais ainda, $M^{-}$é conexo se e somente se a ação de $\pi_{1}\left(\widehat{N}, z^{*}\right)$ sobre $\Omega$ é transitiva.

Demonstração: A condição necessária foi feita na discussão acima assim como a condição necessária e suficiente para a conexidade de $M^{-}$. Basta então demonstrar a condição suficiente do primeiro enunciado. Vamos supor que existe uma ação de $\pi_{1}\left(\widehat{N}, z^{*}\right)$ sobre 
um conjunto $\Omega$ com $d$ elementos tal que as órbitas dadas pela ação de $\left\{v_{i}^{*}\right\}$ e de $\left\{w_{q}^{*}\right\}$ sobre $\Omega$ descrevem as partições $A_{i}$ e $B_{q}$ respectivamente, $i \in I, q \in Q$. Portanto, existe $u$ m revestimento não ramificado $(\widehat{M}, \widehat{\phi}, \widehat{N})$ tal que $\widehat{\phi}^{-1}\left(z^{*}\right)=\Omega .^{6}$ Para cada $i \in I$, as órbitas dadas pela ação de $\left\{v_{i}^{*}\right\}$ sobre $\Omega$ estão em correspondência com as componentes conexas $\widetilde{V}_{i_{j}}^{\circ}$ de $\widehat{\phi}^{-1}\left(V_{x_{i}}^{\circ}\right)$, e para todo $q \in Q$ as órbitas da ação de $\left\{w_{q}^{*}\right\}$ sobre $\Omega$ estão em correspondência com as componentes conexas de $\widehat{\phi}^{-1}\left(C^{q}\right)$. A restrição de $\widehat{\phi}$ sobre cada $\widetilde{V}_{i j}^{\circ}$ é um revestimento não ramificado de $V_{x_{i}}^{\circ}$, e este revestimento está determinado pelo subgrupo $\widehat{\phi}_{*}\left(\pi_{1}\left(\widetilde{V}_{i_{j}}^{\circ}, z_{j_{1}}\right)\right)$ de $\pi_{1}\left(V_{x_{i}}^{\circ}, x_{0_{i}}\right) \cong \mathbb{Z} .^{7}$ Assim nossas únicas opçōes para descrever $\tilde{V}_{i_{j}}^{\circ}$ são os espaços $\{z \in \mathbb{C} \mid 0<\operatorname{Im} z<1\}$ ou $\left\{z \in \mathbb{C}|0<| z \mid<r, r \in \mathbb{Z}^{+}\right\}$. Mas a primeira opção é eliminada pois queremos construir um revestimento ramificado $\left(M^{-}, \phi, N^{-}, B_{\phi}\right)$ e por definição $\phi$ tem que ser uma transformação interior e neste caso a pré-imagem do $x_{i}$ será um conjunto contínuo, o que é impossível. Logo $\widetilde{V}_{i j}^{\circ}$ é homeomorfo a. $\left\{z \in \mathbb{C}|0<| z \mid<r, r \in \mathbb{Z}^{+}\right\}$. Portanto, podemos estender nosso revestimento $\left(\widehat{M}^{-} \widehat{\phi}, \widehat{N}^{-}\right)$para um revestimento ramificado $\left(M^{-}, \phi, N^{-}, B_{\phi}\right)$ simplesmente preenchendo os "buraquinhos" de maneira natural, isto é, levando o "zero" de $\widetilde{V}_{i j}$ no $x_{i}, j \in J_{i}$.

Com as hipóteses e a notação acima o teorema de Hurwitz pode reformular-se assim:

Corolario 2.2 Existe um revestimento ramificado $\left(\mathrm{M}^{-}, \phi, \mathrm{N}^{-}, B_{\phi}\right)$ de grau d tendo $\mathscr{D}$ como dado de ramificação se e somente se é possível definir o homomorfismo $\Psi$. Mais ainda, $\mathrm{M}^{-}$é conexo se e somente se a açăo que corresponde a $\Psi$ é transitiva.

No caso que $N^{-}, M^{-}$sejam superfícies compactas, notemos que tanto $B_{\phi}$ quanto $\phi^{-1}\left(B_{\phi}\right)$ e $d$ são finitos. Em particular se $N^{-}, M^{-}$são superfícies fechadas, isto é compactas e sem bordo.

Proposição 2.3 Se $\phi: M \longrightarrow N$ é um revestimento ramificado de grau d entre superficies fechadas então $\nu(\mathscr{D})=d \chi(N)-\chi(M)$.

Demonstração: Seja $B_{\phi}=\left\{x_{1}, \ldots, x_{k}\right\}$ e $\widehat{M}=\phi^{-1}(\widehat{N})$. Dada uma triangulação para $\widehat{N}$, o levantamento desta induz uma triangulação sobre $\widehat{M}$. Por cada simplexo em $\widehat{N}$

\footnotetext{
${ }^{6}$ Ver [Hu], proposição 1 página 168.

${ }^{7}$ Ver [Ma], teorema 6.6 página 159.
} 
temos exatamente $d$ simplexos em $\widehat{M}$. Isto é

$$
\chi(\widehat{M})=d \chi(\widehat{N})
$$

$\operatorname{Mas} \chi(\widehat{N})=\chi(N)-k$ e $\chi(\widehat{M})=\chi(M)-\sum_{i=1}^{k}\left(d-\nu\left(A_{i}\right)\right)=\chi(M)-d k-\nu(\mathscr{D}), \log \mathrm{o}$ substituindo em $(2.2)$ temos que $\nu(\mathscr{D})=d \chi(N)-\chi(M)$.

A igualdade da proposição anterior é conhecida como a fórmula de Riemann-Hurwitz. Se $N$ é fechada as opções são:

$$
N= \begin{cases}\mathbb{S}^{2} & \text { Esfera } \\ \mathbb{T}_{n} & \text { Soma conexa de } n \text { toros } \\ \mathbb{R P}_{n} & \text { Soma conexa de } n \text { planos projetivos } \mathbb{R} \mathrm{P}^{2}\end{cases}
$$

portanto

$$
\pi_{1}\left(\widehat{N}, z^{*}\right)= \begin{cases}\left\langle\left\{v_{1}^{*}\right\}, \ldots,\left\{v_{k}^{*}\right\} \mid\left\{v_{1}^{*}\right\} \ldots\left\{v_{k}^{*}\right\}=1\right\rangle & N=\mathbb{S}^{2} \\ \left\langle\left\{v_{1}^{*}\right\}, \ldots,\left\{v_{k}^{*}\right\}, \beta_{1}, \gamma_{1}, \ldots, \beta_{n}, \gamma_{n} \mid\left\{v_{1}^{*}\right\} \ldots\left\{v_{k}^{*}\right\}\left[\beta_{1}, \gamma_{1}\right] \ldots\left[\beta_{n}, \gamma_{n}\right]=1\right\rangle & N=\mathbb{T}_{n} \\ \left\langle\left\{v_{1}^{*}\right\}, \ldots,\left\{v_{k}^{*}\right\}, \beta_{1}, \ldots, \beta_{n} \mid\left\{v_{1}^{*}\right\} \ldots\left\{v_{k}^{*}\right\} \beta_{1}^{2} \ldots \beta_{n}^{2}=1\right\rangle & N=\mathbb{R}_{n}\end{cases}
$$

assim, a construção de $\Psi$ depende da relação $r$ em cada grupo fundamental.

Notemos que quando é possivel definir $\Psi$, em cada um dos casos $\Psi(r)=1$ e a adaptação do teorema de Hurwitz a esta situação é a seguinte:

Corolario 2.4 Seja $N$ uma superficie fechada $e \mathscr{D}=\left\{A_{1}, \ldots, A_{k}\right\}$ uma coleçăo de partições de d (onde é possivel ter repetições). Então $\mathscr{D}$ é realizável se e somente se existe $\sigma_{x_{i}} \in S_{d}$ tal que o conjunto de comprimentos dos seus ciclos determinam a partição $A_{i}$, $i=1, \ldots, k, e$ :

1. Para $N=\mathbb{S}^{2}, \sigma_{x_{1}} \ldots \sigma_{x_{k}}=1$ e $\left\langle\sigma_{x_{1}}, \ldots, \sigma_{x_{k}}\right\rangle$ age transitivamente sobre $\{1, \ldots, d\}$.

2. Para $N=\mathbb{T}_{n}$, existem $\widehat{\beta}_{j}, \widehat{\gamma}_{j} \in S_{d}, 1 \leq j \leq n$, tais que

$\sigma_{x_{1}} \ldots \sigma_{x_{k}}\left[\widehat{\beta}_{1}, \widehat{\gamma}_{1}\right] \ldots\left[\widehat{\beta}_{n}, \widehat{\gamma}_{n}\right]=1 \in\left\langle\sigma_{x_{1}}, \ldots, \sigma_{x_{k}}, \widehat{\beta}_{1}, \widehat{\gamma}_{1}, \ldots, \widehat{\beta}_{n}, \widehat{\gamma}_{n}\right\rangle$ age transitivamente sobre $\{1, \ldots, d\}$.

3. Para $N=\mathbb{R} \mathrm{P}_{n}$, existem $\widehat{\beta}_{j} \in S_{d}, 1 \leq j \leq n$, tais que $\sigma_{x_{1}} \ldots \sigma_{x_{k}} \widehat{\beta}_{1}^{2} \ldots \widehat{\beta}_{n}^{2}=1 \epsilon$ $\left\langle\sigma_{x_{1}}, \ldots, \sigma_{x_{k}}, \widehat{\beta}_{1}, \ldots, \widehat{\beta}_{n}\right\rangle$ age transitivamente sobre $\{1, \ldots, d\}$. 
Define-se o Revestimento Orientável Duplo de $N, \varphi: \tilde{N} \longrightarrow N, N=\mathbb{R} \mathrm{P}_{n}$, como o revestimento não ramificado que corresponde ao subgrupo de $\pi_{1}\left(N, z^{*}\right)$ que é o kernel do homomorfismo

$$
\begin{aligned}
\omega: \pi_{1}\left(N, z^{*}\right) & \longrightarrow\{+1,-1\} \\
\beta_{i} & \longmapsto-1
\end{aligned}
$$

para $1 \leq i \leq n$. Analogamente o revestimento orientável duplo de $\widehat{N}$, é o revestimento não ramificado que corresponde ao kernel do homomorfismo

$$
\begin{aligned}
\widehat{\omega}: \pi_{1}\left(\widehat{N}, z^{*}\right) & \longrightarrow\{+1,-1\} \\
\left\{v_{i}^{*}\right\} & \longmapsto+1 \\
\beta_{j} & \longmapsto-1
\end{aligned}
$$

para $1 \leq i \leq k, 1 \leq j \leq n$

Por outro lado, se $\Psi: \pi_{1}\left(\widehat{N}, z^{*}\right) \longrightarrow S_{d}$ é a representação de Hurwitz associada a $\left(M, \phi, N, B_{\phi}\right)$, então o revestimento não ramificado $\widehat{\phi}: \widehat{M} \longrightarrow \widehat{N}, \widehat{\phi}=\left.\phi\right|_{\widehat{M}}$, corresponde ao subgrupo $\Psi^{-1}\left(S_{d-1}\right) \subset \pi_{1}(\widehat{N})$. Veja, o revestimento $\widehat{\phi}: \widehat{M} \longrightarrow \widehat{N}$ corresponde ao subgrupo $\widehat{\phi}_{*}\left(\pi_{1}\left(\widehat{M}, z_{1}\right)\right) \subset \pi_{1}\left(\widehat{N}, z^{*}\right), z_{1} \in \widehat{\phi}^{-1}\left(z^{*}\right),{ }^{8}$ assim, se $\alpha \in \widehat{\phi}_{*}\left(\pi_{1}\left(\widehat{M}, z_{1}\right)\right)$, existe $\beta \in \pi_{1}\left(\widehat{M}, z_{1}\right)$ tal que $\widehat{\phi}_{*}(\beta)=\alpha$. Isto é, $\widetilde{\alpha}(0)=\widetilde{\alpha}(1)=z_{1}$, onde $\widetilde{\alpha}$ é o único levantamento de $\alpha$ tendo $z_{1}$ como ponto inicial. E segundo a ação que o revestimento determina, isto quer dizer que $\alpha$ pertence à isotropia de $z_{1}$, que denotamos por $I_{\pi_{1}\left(\hat{N}, z^{*}\right)}\left(z_{1}\right)$. Donde $\widehat{p h i} i_{*}\left(\pi_{1}\left(\widehat{M}, z_{1}\right)\right)=I_{\pi_{1}\left(\widehat{N}, z^{*}\right)}\left(z_{1}\right)=\left\{\alpha \in \pi_{1}\left(\widehat{N}, z^{*}\right) \mid z_{1} \cdot \alpha=z_{1}\right\}$. Logo, se $\alpha \in I_{\pi_{1}\left(\widehat{N}, z^{*}\right)}\left(z_{1}\right)$ então $\Psi(\alpha) \in S_{d-1}$.

Lema 2.5 Seja $\phi: M \longrightarrow N$ é um revestimento ramificado conexo de grau de $N=\mathbb{R}_{n}$. $M$ é orientável se e somente se $\Psi^{-1}\left(S_{d-1}\right) \subset \operatorname{Ker} \widehat{\omega}$.

Notemos que o homomorfismo $\Psi$ determinado por $\left(M, \phi, N, B_{\phi}\right)$ não é único. De fato, $\phi$ determina um conjunto de classes de equivalencia de homomorfismos. Vamos dizer que dois homomorfismos $\Psi$ e $\Gamma$ são equivalentes se existe uma permutação $\sigma \in S_{d}$ tal que para

\footnotetext{
${ }^{8}$ Ver [Ma], teorema 10.2 página 175.
} 
todo $b \in \pi_{1}\left(\widehat{N}, z^{*}\right), \Gamma(b)=\sigma^{-1} \Psi(b) \sigma$. Isto significa que para todo $b \in \pi_{1}\left(\widehat{N}, z^{*}\right), \Psi(b) \mathrm{e}$ $\Gamma(b)$ têm a mesma estrutura cíclica, e que vamos obter $\Gamma(b)$ simplesmente aplicando $\sigma$ nos símbolos de $\Psi(b)$. Logo, renomeando os símbolos de $\phi^{-1}\left(z^{*}\right), \phi$ induz $\Gamma$.

Suponhamos que $\phi: M \longrightarrow N$ e $\theta: L \longrightarrow N$ são revestimentos ramificados de grau $d$ sobre $N \operatorname{com} B_{\phi}=B_{\theta}$, e tais que induzem representações de Hurwitz equivalentes. Sejam $\phi^{-1}\left(z^{*}\right)=\left\{m_{1}, \ldots, m_{d}\right\}, \theta^{-1}\left(z^{*}\right)=\left\{l_{1}, \ldots, l_{d}\right\}$ e $M_{1}$ uma componente conexa de $M$. Vamos supor que $\left\{m_{1}, \ldots, m_{d_{1}}\right\}$ são as preimagens de $z^{*}$ em $M_{1}$. Já que $\phi$ e $\theta$ induzem representações de Hurwitz equivalentes, existe $\sigma \in S_{d}$ tal que $\left\{l_{\sigma(1)}, \ldots, l_{\sigma\left(d_{1}\right)}\right\}$ são as preimagens de $z^{*}$ na componente conexa $L_{1}$ de $L$. Assim, os revestimentos $\widehat{\phi}_{1}: \widehat{M}_{1} \longrightarrow \widehat{N}$ e $\widehat{\theta}_{1}: \widehat{L}_{1} \longrightarrow \widehat{N}$ são equivalentes. ${ }^{9}$ Para demonstrar este fato, precisamos mostrar que existe um homeomorfismo $\mu: \widehat{M}_{1} \longrightarrow \widehat{L}_{1}$ tal que o diagrama

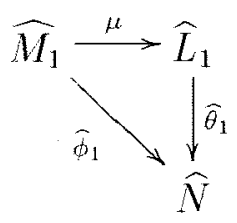

comute. Mas isto equivale a demonstrar que $\widehat{\phi}_{1 *}\left(\pi_{1}\left(\widehat{M}_{1}, m_{1}\right)\right)=\widehat{\theta}_{1 *}\left(\pi_{1}\left(\widehat{L_{1}}, l\right)\right),{ }^{10}$ para $\operatorname{algum} l \in\left\{l_{\sigma(1)}, \ldots, l_{\sigma\left(d_{1}\right)}\right\}$. Vejamos:

$$
\begin{aligned}
\widehat{\phi}_{1 *}\left(\pi_{1}\left(\widehat{M}_{1}, m_{1}\right)\right) & =\left\{\beta \in \pi_{1}\left(\widehat{N}, z^{*}\right) \mid m_{1} \cdot \beta=m_{1}\right\} \\
& =\left\{\beta \in \pi_{1}\left(\widehat{N}, z^{*}\right) \mid \Psi(\beta)(1)=1\right\} \\
& =\left\{\beta \in \pi_{1}\left(\widehat{N}, z^{*}\right) \mid \sigma \Gamma(\beta) \sigma^{-1}(1)=1\right\} \\
& =\left\{\beta \in \pi_{1}\left(\widehat{N}, z^{*}\right) \mid \Gamma(\beta)\left(\sigma^{-1}(1)\right)=\sigma^{-1}(1)\right\} \\
& =\widehat{\theta}_{1 *}\left(\pi_{1}\left(\widehat{L_{1}}, l_{\sigma^{-1}(1)}\right)\right) .
\end{aligned}
$$

Usando uma técnica similar à usada na demonstração do teorema de Hurwitz, podemos estender tanto $\widehat{\phi_{1}}$ quanto $\widehat{\theta_{1}}$ a revestimentos ramificados $\phi_{1}$ e $\theta_{1}$ de $M_{1}$ e $L_{1}$ sobre $N$ respectivamente. Além disso, existe uma bijeção entre as componentes conexas de $M$ e de $L$. Portanto se $\phi$ e $\theta$ induzem representações de Hurwitz equivalentes, $\phi$ e $\theta$ são

\footnotetext{
${ }^{9}$ O chapéu sobre as superfícies é para denotar as superfícies depois de furadas e, sobre as funçoes, é para denotar a restrição a elas.

${ }^{10}$ Ver [Ma], corolário 6.4 página 159.
} 
equivalentes, isto é, existe um homeomorfismo $h: M \longrightarrow L$ tal que o diagrama

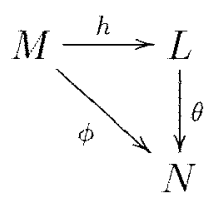

comuta.

Reciprocamente, se $\phi$ e $\theta$ são revestimentos ramificados equivalentes, eles determinam representações de Hurwitz $\Psi, \Gamma$ equivalentes. Pois, que $\phi$ e $\theta$ sejam equivalentes implica a existência de um homeomorfismo $h$ que faz comutar o diagrama acima. Logo o diagrama restrito a $\widehat{M}, \widehat{L}$ e $\widehat{N}$ também comuta. Além disso, notemos que $h$ determina uma bijeção entre os conjuntos $\left\{m_{1}, \ldots, m_{d}\right\}$ e $\left\{l_{1}, \ldots, l_{d}\right\}$, portanto podemos considerar esta restrição de $h$ como uma permutação de um conjunto com $d$ elementos. Além disso, para todo $\beta \in \pi_{1}\left(\widehat{N}, z^{*}\right)$ existem $i, j \in\left\{1, \ldots, d_{1}\right\}$ tais que $m_{i} \cdot \beta=m_{j}$, donde $h\left(m_{i}\right) \cdot h(\beta)=h\left(m_{j}\right)$ e pela unicidade dos levantamentos isto que dizer que $h^{-1} \Gamma(\beta) h(i)=j$. Assim $\Psi$ e $\Gamma$ são equivalentes.

Seja $\mathfrak{F}$ o conjunto de todas as classes de equivalência de revestimentos ramificados de grau $d$ com seus pontos de ramificação localizados em $X=\left\{x_{1}, \ldots, x_{k}\right\} \subset N$, e seja $\mathfrak{P}$ o conjunto das classes de equivalência de homomorfismos entre $\pi_{1}\left(\widehat{N}, z^{*}\right)$ e $S_{d}$. Vamos mostrar que existe uma bijeção $\Lambda$ entre estes conjuntos, $\Lambda: \mathfrak{F} \longrightarrow \mathfrak{P}$. Já mostramos que $\Lambda$ é injetora. Para mostrar que é sobrejetora, usaremos algumas técnicas de construção de Hurwitz.

Seja o homomorfismo $\Psi: \pi_{1}\left(\widehat{N}, z^{*}\right) \longrightarrow S_{d}$. Vamos construir um revestimento ramificado de grau $d$ sobre $N, \phi: M \longrightarrow N$, tal que $\phi$ vai induzir $\Psi$. Consideremos a representaçăo poligonal de $N$ dada na figura 2.1 , onde $n \geq 0$ é o gênero da superfície, os $b_{i} \in \beta_{i}, c_{i} \in \gamma_{i}$ e os $y_{j}$ 's são caminhos que unem $z^{*} \operatorname{com} x_{i}, i=1, \ldots, k .^{11}$ Recortando pela trajetória de cada $y_{i}$ obtemos um disco fechado $Z$ (Ver Figura 2.2) que orientamos no sentido horário.

Vamos construir $M$ mediante a colagem de $d$ copias de $Z, Z_{1}, \ldots, Z_{d}$, segundo as seguintes identificações: se $w$ é uma aresta sobre o bordo de $Z$ tal que a permutação

\footnotetext{
${ }^{11} \beta_{i}, \gamma_{i}$ geradores do grupo fundamental de $N$ dependendo do caso.
} 


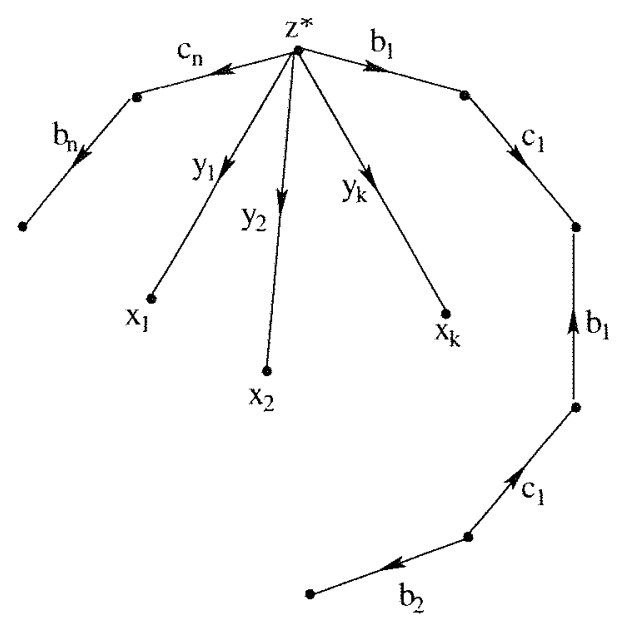

$\mathrm{N}$ orientável

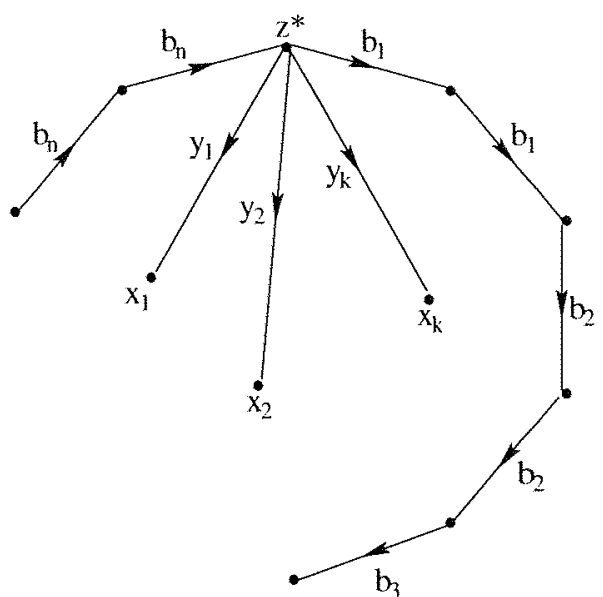

$\mathrm{N}$ não orientável

Figura 2.1: Representação Poligonal

$\Psi(\{w\})$ leva o símbolo $i$ no símbolo $j,{ }^{12}$ colamos $Z_{i}$ a $Z_{j}$ identificando as arestas $w^{i}$ e $w^{j^{-1}}$ onde os índices indicam o disco ao qual elas pertencem. Defina o revestimento ramificado

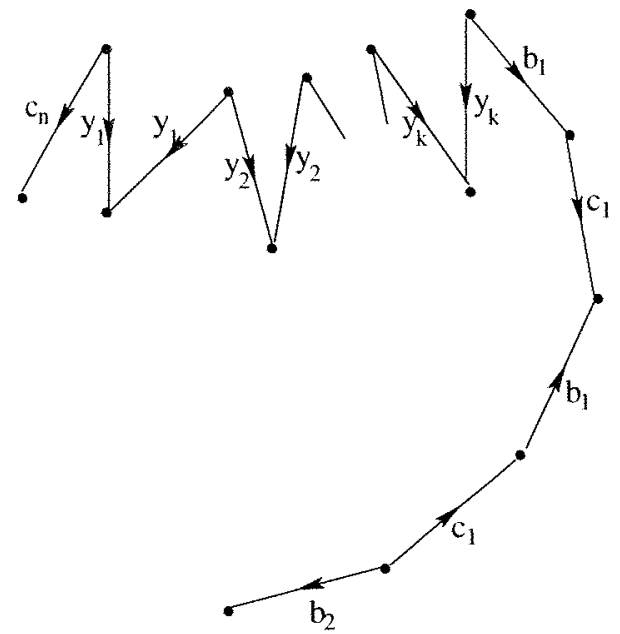

Norientável

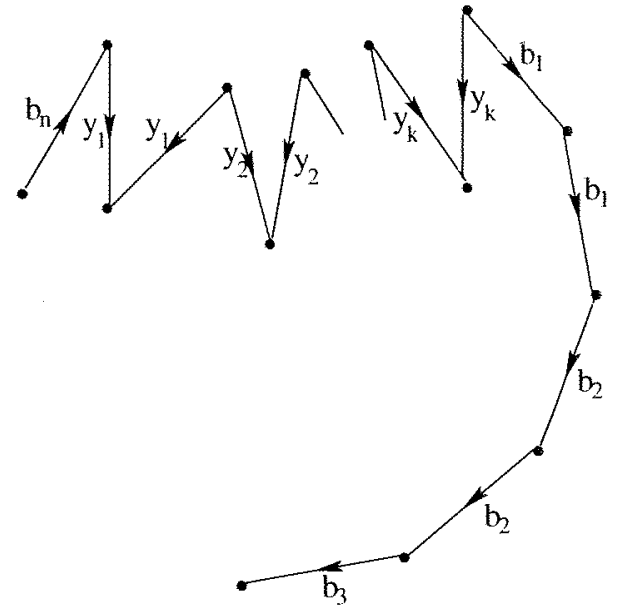

N não orientável

Figura 2.2: Disco fechado Z

$\phi: M \longrightarrow N$ usando os homeomorfismos $\phi_{i}: Z_{i} \longrightarrow Z, i=1, \ldots, d$. Podemos nomear as pré-imagens de $z^{*}$ tal que $\phi$ induz $\Psi$, pois construímos $\phi$ partindo de $\Psi$.

Da construção, a possível localização dos pontos de ramificação está sobre $z^{*}$ e os $x_{i}$,

\footnotetext{
${ }^{12}$ Se $w=y_{t}$, consideramos $w \in\left\{v_{t}^{*}\right\}$.
} 
$i=1, \ldots, k$. Sabemos que o defeito de cada $x_{i}$ está determinado pelo número de ciclos na decomposição da permutação $\Psi\left(\left\{v_{i}^{*}\right\}\right)$ e, pela teoria já estudada, este número é menor que $d$. Por outro lado, o bordo $r$ de $Z$ é um laço em $z^{*}$ e $\Psi(r)=1, \operatorname{logo} \Psi(r)$ tem $d$ ciclos e portanto $z^{*}$ tem $d$ pré-imagens. Assim, os pontos de ramificação se localizam somente sobre os $x_{i}$.

Proposição 2.6 Seja $\phi: M \longrightarrow N$ um revestimento ramificado conexo de grau d entre superficies fechadas. Então:

(1) Se $N$ é orientável, $M$ é orientável.

(2) Se dé impar e $N$ não orientável, $M$ é não orientável.

Demonstração: Seja $\Psi$ um homomorfismo induzido por $\phi$. Pela construção na discussão acima, partindo de $\Psi$ podemos construir um revestimento ramificado $\theta: L \longrightarrow N$ de grau $d$ que induz $\Psi$. Mas já provamos que isto implica a existência de um homeomorfismo $h$ que faz comutar o diagrama (2.3). Da construção temos que $L$ é orientável, portanto $M$ é orientável, e está provado (1). Para (2), pelo lema 2.5 temos que $M$ é não orientável se e somente se existe um $\alpha \in \Psi^{-1}\left(S_{d-1}\right)$ tal que na sua representação como produto de $\left\{v_{i}^{*}\right\}$ 's e $\beta_{j}$ 's aparece um número ímpar dos últimos, levando em consideração suas potências. Se $d$ é ímpar, em particular $\Psi\left(\beta_{j}\right)$ é uma permutação de um número ímpar de símbolos e na sua decomposição cíclica existe no mínimo um ciclo de comprimento $p$ ímpar. Seja $\left(x_{1} \ldots x_{p}\right)$ este ciclo, $\log o \beta_{j}^{p}$ fixa $x_{1}$ e portanto $\beta_{j}^{p} \in \Psi^{-1}\left(S_{d-1}\right), \operatorname{logo} M$ é não orientável.

Corolario 2.7 Se $\left(M, \phi, N, B_{\phi}\right)$ é um revestimento ramificado de grau d tendo $\mathscr{D}$ como dado de ramificação, então:

$$
\nu(\mathscr{D}) \geq \begin{cases}2 d-2 & \text { se } N=\mathbb{S}^{2} \\ d-2 & \text { se } N=\mathbb{R} \mathrm{P}^{2} \in M \text { é orientável } \\ d-1 & \text { se } N=\mathbb{R} \mathrm{P}^{2} \text { e } M \text { é não orientável }\end{cases}
$$

Demonstração: Se $M$ é orientável, $\chi(M) \leq 2=\chi\left(\mathbb{S}^{2}\right)$. Se $M$ é não orientável, $\chi(M) \leq$ $1=\chi\left(\mathbb{R} P^{2}\right)$. Agora é só aplicar a fórmula de Riemann-Hurwitz. 


\section{Capítulo 3}

\section{Sobre o Grupo Simétrico}

O objetivo deste capítulo é apresentar a teoria sobre o grupo simétrico necessária para avançar no estudo do problema de realização. Resultados disponíveis em [BGKZ2], [EKS] $\mathrm{e}[\mathrm{Ez}]$.

\subsection{Resultados Básicos}

Para começar lembremos ${ }^{1}$ que toda permutação $\alpha \in S_{d}$ pode ser escrita de um único jeito como produto de ciclos disjuntos $\gamma_{i}$

$$
\alpha=\gamma_{1} \ldots \gamma_{r}
$$

incluindo ciclos de comprimento um se for necessário. Portanto cada $\alpha \in S_{d}$ determina uma partição $A=\|\alpha\|=\left[\left|\gamma_{1}\right|, \ldots,\left|\gamma_{r}\right|\right]$ de $d$, dada pelos comprimentos $\left|\gamma_{i}\right|$ de cada um de seus ciclos. Vamos dizer que uma permutação $\beta \in S_{d}$ realiza a partição $A$ ou que $\beta \in A$, se a coleção dos comprimentos dos ciclos de $\beta$ está dada por $A$.

Por outro lado, toda permutação pode ser escrita como produto de transposições onde o número de termos nesta decomposição é invariante módulo 2.

Tem-se que uma permutação é par se o número de termos em alguma decomposição

\footnotetext{
${ }^{1}$ Ver [DM] capítulo 1, [Ro] capítulo 3 e [Wi] capítulo 1.
} 
desta como produto de transposições é congruente a zero módulo 2, caso contrário a permutação é ímpar. Além disso, o produto de duas transposições é par se e somente se ambas têm a mesma paridade.

Vamos supor que cada ciclo $\gamma_{i}$ em (3.1) tem comprimento $a_{i}, \gamma_{i}=\left(r_{i_{1}} \ldots r_{i_{i}}\right)$. É claro que a decomposição

$$
\gamma_{i}=\left(r_{i_{1}} r_{i_{2}}\right)\left(r_{i_{1}} r_{i_{3}}\right) \ldots\left(r_{i_{1}} r_{i_{a_{i}}}\right)
$$

dá conta da paridade de $\gamma_{i}$, pois $\gamma_{i}$ vai ser par se e somente se $a_{i}$ é ímpar. Daqui é claro que a paridade de $\alpha$ depende da paridade de cada $\gamma_{i}$, e já que estes ciclos são disjuntos, é possível fatorar $\alpha$ como produto de transposições do tipo (3.2). Portanto $\alpha$ é par se e somente se

$$
\sum_{i=1}^{r}\left(a_{i}-1\right) \equiv 0 \quad(\bmod 2)
$$

\subsection{Produtos em $S_{d}$}

Seja $d \in \mathbb{Z}^{+}$e $\pi(d)$ o conjunto de partições de $d$ onde cada termo das partições é maior que zero.

Se $A=\left[a_{1}, \ldots, a_{r}\right] \in \pi(d)$, vamos definir $\nu(A)=\sum_{i=1}^{r}\left(a_{i}-1\right)=d-r$. Mais ainda, se $\alpha \in A$, definimos $\nu(\alpha)=\nu(A)$.

Proposição 3.2.1 Se $\alpha \in S_{d} \operatorname{com} \nu(\alpha)=d-r$, então para todo $t \geq 0$ tal que $r+2 t \leq d$, $\alpha$ pode-se escrever como produto de um d-ciclo $\epsilon$ um $(r+2 t)$-ciclo.

Demonstração: Sem perda de generalidade, tomemos

$$
\alpha=\left(1 \ldots a_{1}\right)\left(a_{1}+1 \ldots a_{2}\right) \ldots\left(a_{r-1}+1 \ldots a_{r}\right)
$$

onde $1 \leq a_{1}<a_{2}<\cdots<a_{r}=d$. Seja

$$
\beta=\left(\begin{array}{llll}
1 & a_{1}+1 & a_{2}+1 \ldots a_{r-1}+1 & b_{1} \ldots b_{2 t}
\end{array}\right)
$$


onde $b_{1}<b_{2}<\cdots<b_{2 t}$ e não estão no conjunto $\left\{1, a_{1}+1, \ldots, a_{r-1}+1\right\}$. Então $\gamma=\alpha \beta$ é um $d$-ciclo: seja $\beta_{0}=\left(\begin{array}{ll}1 & a_{1}+1 \ldots a_{r-1}+1\end{array}\right)$ e $\beta_{1}=\left(\begin{array}{ll}1 & b_{1} \ldots b_{2 t}\end{array}\right) \operatorname{assim} \beta=\beta_{0} \beta_{1}, \operatorname{logo}$

$$
\begin{aligned}
& \alpha \beta=\alpha \beta_{0} \beta_{1} \\
& =\left(\begin{array}{lll}
1 & 2 \ldots d
\end{array}\right)\left(\begin{array}{ll}
1 & b_{1} \ldots b_{2 t}
\end{array}\right) \\
& =\left(1 \ldots b_{1}-1 \quad b_{2} \ldots b_{3}-1 \quad b_{4} \ldots b_{2 t-1}-1\right. \\
& \left.b_{2 t} \ldots d \quad b_{1} \ldots b_{2}-1 \quad b_{3} \ldots b_{2 t-2}-1 \quad b_{2 t-1} \ldots b_{2 t}-1\right) \text {. }
\end{aligned}
$$

Donde $\alpha=\gamma \beta^{-1}$ tem a estrutura procurada.

Corolario 3.2.2 Toda permutação par de $S_{d}$ pode ser escrita como:

1. um produto de dois d-ciclos,

2. um comutador $\alpha \beta \alpha^{-1} \beta^{-1}$ com $\alpha$ d-ciclo,

3. um produto de dois quadrados $\alpha^{2} \beta^{2}$ com $\alpha \beta$ d-ciclo.

Demonstração: Se $\gamma$ é uma permutação par então por (3.3) $\nu(\gamma)=d-r$ é par, o que implica que $d$ e $r$ têm a mesma paridade e portanto $d=r+2 t, t>0$. Pela proposição 3.2 .1 existem $d$-ciclos $\sigma, \tau$ tais que $\gamma=\sigma \tau$ e a afirmação 1 está resolvida. Do anterior, $\tau \mathrm{e}$ $\sigma^{-1}$ são conjugados, isto é, existe $\beta \in S_{d}$ tal que $\tau=\beta \sigma^{-1} \beta^{-1}$ donde $\gamma=\sigma \beta \sigma^{-1} \beta^{-1}$ com $\sigma d$-ciclo e a afirmação 2 está pronta. Finalmente para mostrar a terceira, já que $\sigma$ e $\tau$ são conjugados, existe $\alpha \in S_{d}$ tal que $\sigma=\alpha \tau \alpha^{-1} \operatorname{logo} \gamma=\left(\alpha \tau \alpha^{-1}\right) \tau=\alpha \alpha \alpha^{-1} \tau \alpha^{-1} \tau=$ $\alpha^{2}\left(\alpha^{-1} \tau\right)^{2}$ e $\alpha\left(\alpha^{-1} \tau\right)$ é um $d$-ciclo.

Proposição 3.2.3 Se $\alpha, \beta \in S_{d}$ são d-ciclos, existe $\gamma \in S_{d}$ fixando um elemento tal que $\alpha=\gamma \beta \gamma^{-1}$.

Demonstração: Sejam $\alpha=\left(c_{1} \ldots c_{d}\right)$ e $\beta=\left(t_{1} \ldots t_{d}\right)$. Se $c_{i}=t_{j}$ é só fazer

$$
\gamma=\left(\begin{array}{ccccc}
c_{1} & \ldots & c_{i} & \ldots & c_{d} \\
\ldots & t_{j-1} & t_{j} & t_{j+1} & \ldots
\end{array}\right)
$$


Lema 3.2.4 Sejam $A, B \in \pi(d) \operatorname{com} \nu(A)+\nu(B)=d-t, t \geq 1$. Para todo $\alpha \in A, \beta \in B$ tem-se $\nu(\alpha \beta) \leq d-t$.

Demonstração: Sejam $\alpha \in A, \beta \in B$ e $\gamma=\alpha \beta$. Suponha que a ação do grupo $\langle\alpha, \beta\rangle$ sobre $\{1, \ldots d\}$ determina exatamente $q$ órbitas. Já que $\gamma \in\langle\alpha, \beta\rangle, \gamma$ determina no mínimo $q$ órbitas, isto é, $\nu(\gamma) \leq d-q$. Se $C$ denota a partição dada por $\gamma$ então

$$
\nu(A)+\nu(B)+\nu(C) \leq d-t+d-q=2 d-t-q .
$$

Por outro lado, $\alpha \beta \gamma^{-1}=1$, e tirando a conexidade citada no corolário 2.4 já que $\langle\alpha, \beta\rangle$ não age transitivamente sobre $\{1, \ldots, d\}$, existe um revestimento ramificado $\phi: M \longrightarrow \mathbb{S}^{2}$ com dado de ramificação $\{A, B, C\}$ e tal que $M$ é uma superfície fechada com exatamente $q$ componentes conexas. Pela fórmula de Riemann-Hurwitz

$$
\nu(A)+\nu(B)+\nu(C)=d \chi\left(\mathbb{S}^{2}\right)-\chi(M)
$$

$\operatorname{mas} \chi(M) \leq q \chi\left(\mathbb{S}^{2}\right)=2 q, \log 0$

$$
2 d-2 q \leq \nu(A)+\nu(B)+\nu(C) \leq 2 d-t-q
$$

daqui $q \geq t$ donde $\nu(\gamma) \leq d-q \leq d-t$.

Proposição 3.2.5 Sejam $A, B \in \pi(d) \operatorname{com} \nu(A)+\nu(B)=d-t, t \geq 1$. Então existem. $\alpha \in A$ e $\beta \in B$ tais que:

1. a ação do grupo $\langle\alpha, \beta\rangle$ determina precisamente tórbitas e,

2. se $C$ denota a partiçăo dada pela ação descrita no numeral anterior então $\alpha \beta \in C$.

Demonstração: A segunda afirmação é consequência da primeira. Veja, pelo lema 3.2.4 $\nu(\alpha \beta) \leq d-t$ e na prova do mesmo vamos ter que

$$
2 d-2 t \leq \nu(\alpha)+\nu(\beta)+\nu(\alpha \beta) \leq 2 d-2 t
$$

pois $q=t, \log 0$

$$
\begin{aligned}
\nu(\alpha)+\nu(\beta)+\nu(\alpha \beta) & =2 d-2 t \\
d-t+\nu(\alpha \beta) & =2 d-2 t \\
\nu(\alpha \beta) & =d-t .
\end{aligned}
$$


Já que a partição associada a $\alpha \beta$ deve refinar a partição $C$ e $\nu(\alpha \beta)=\nu(C)$, então $\alpha \beta \in C$.

Provaremos a afirmação 1 fazendo indução sobre $d$. Para $d=1$ é trivial. Para $d=2$ as opções de $t$ são $t=1$ ou $t=2$. Se $t=1, \nu(A)+\nu(B)=1$, o que implica que $\nu(A)=0$ ou $\nu(B)=0$. Sem perda de generalidade vamos supor que $\nu(A)=0$, então $\alpha \in A$ é a identidade, logo $\langle\alpha, \beta\rangle=\langle\beta\rangle$ determina $d-\nu(B)=1$ órbitas, pois $\beta \in B$. Se $t=2, \nu(A)+\nu(B)=0, \operatorname{logo} \alpha=\beta=1$ que obviamente age determinando 2 órbitas. Procedendo indutivamente, suponhamos $\nu(A)=d-r>0$. Fixemos um representante $\alpha \in A=\left[d_{1}, \ldots, d_{r}\right]$

$$
\alpha=\left(\begin{array}{lll}
1 & 2 \ldots d_{1}
\end{array}\right)\left(d_{1}+1 \ldots d_{2}\right) \ldots\left(d_{r-1}+1 \ldots d_{r}\right)
$$

onde $d_{r}=d$ e $d_{1}>1$. Seja $B=\left[b_{1}, b_{2}, \ldots, b_{s}\right]$, portanto $\nu(B)=d-s$ e $t=r+s-d$. Seja $\beta \in B, \beta=\beta_{1} \ldots \beta_{s}$, tal que os $\beta_{i}$ são ciclos disjuntos que representam cada um dos $b_{i}$-ciclos cujos termos ainda não determinamos, $i=1, \ldots, s$.

Observe que $b_{1} \leq r$, já que

$$
d-1 \geq \nu(A)+\nu(B) \geq(d-r)+\left(b_{1}-1\right)
$$

donde $r \geq b_{1}$. Seja $\beta_{1}=\left(\begin{array}{ll}1 & d_{1}+1 \ldots d_{b_{1}-1}+1\end{array}\right)$. As órbitas dadas pela ação de $\langle\alpha\rangle$ sobre $\{1, \ldots, d\}$ são os conjuntos

$$
P_{i}=\left\{d_{i-1}+1, \ldots, d_{i}\right\}
$$

$i=1, \ldots, r$ e $d_{0}=0$. E pela escolha de $\beta_{1}$, o conjunto $P_{1} \cup \ldots \cup P_{b_{1}}$ vai estar contido numa mesma órbita dada por $\langle\alpha, \beta\rangle$. Os $d-b_{1}$ pontos de $\{1, \ldots, d\}-\left\{\right.$ elementos de $\left.\beta_{1}\right\}$ ainda não distribuídos nos ciclos $\beta_{2}, \ldots, \beta_{s}$, podem dividir-se em $r-b_{1}+1$ conjuntos $Q_{1}, \ldots, Q_{r-b_{1}+1}$ onde:

$$
\begin{aligned}
Q_{1} & =\left\{1, \ldots, d_{b_{1}}\right\}-\left\{\text { elementos de } \beta_{1}\right\} \\
Q_{2} & =P_{b_{1}+1} \\
& \vdots \\
Q_{r-b_{1}+1} & =P_{r} .
\end{aligned}
$$

Note que $Q_{1} \neq \emptyset$ já que $d_{1}>1$. Façamos agora $A^{\prime}=\left[\left|Q_{1}\right|, \ldots,\left|Q_{r-b_{1}+1}\right|\right]$ e $B^{\prime}=\left[b_{2}, \ldots, b_{s}\right]$ elementos de $\pi\left(d-b_{1}\right)$, onde $\left|Q_{i}\right|$ denota a cardinalidade do conjunto $Q_{i}$. Logo, $\nu\left(A^{\prime}\right)=$ 
$\left(d-b_{1}\right)-\left(r-b_{1}+1\right)$ donde $\nu\left(A^{\prime}\right)=d-r-1$ e $\nu\left(B^{\prime}\right)=\left(d-b_{1}\right)-(s-1)$. Assim,

$$
\begin{aligned}
\nu\left(A^{\prime}\right)+\nu\left(B^{\prime}\right) & =d-r-1+d-b_{1}-s+1 \\
& =2 d-r-b_{1}-s \\
& =\left(d-b_{1}\right)+d-r-s \\
& =\left(d-b_{1}\right)-t .
\end{aligned}
$$

Seja $\alpha^{\prime}=\alpha_{1}^{\prime} \ldots \alpha_{r-b_{1}+1}^{\prime} \in A^{\prime}, \alpha_{i}^{\prime} \in Q_{i}$. Por indução, existe $\beta^{\prime} \in B^{\prime}$ com elementos em $\{1, \ldots, d\}-\left\{\right.$ elementos de $\left.\beta_{1}\right\}$ tal que $\left\langle\alpha^{\prime}, \beta^{\prime}\right\rangle$ determina $t^{\prime}$ órbitas, onde $t^{\prime}=\left(d-b_{1}\right)-$ $\nu\left(A^{\prime}\right)-\nu\left(\beta^{\prime}\right)=t$. Logo é só fazer $\beta=\beta_{1} \beta^{\prime}$ para completar a prova, pois se $x \in\{1, \ldots, d\}$ pode acontecer:

(1) $x \geq d_{b_{1}}+1$, e neste caso a órbita de $x$ está determinada pela ação dos $\alpha_{t}^{\prime}, t>1$ e de $\beta^{\prime}$.

(2) $x<d_{b_{1}}+1$, neste caso $x$ pertence à mesma órbita dos elementos em $\{1, \ldots, d\}-$ $\left\{\right.$ elementos de $\left.\beta_{1}\right\}$ menores ou iguais que $d_{b_{1}}$, assim $\langle\alpha, \beta\rangle$ determina $t$ órbitas.

Proposição 3.2.6 Sejam $A, B \in \pi(d) \operatorname{com} \nu(A)+\nu(B)=(d-1)+r, r>0$. Então para cada $k, 0 \leq k \leq r, k \equiv r(\bmod 2)$, podemos escolher $\alpha \in A, \beta \in B$ tais que o grupo $\langle\alpha, \beta\rangle$ age transitivamente sobre $\{1, \ldots, d\}$ e $\nu(\alpha \beta)=(d-1)-k$.

Demonstração: Seja $B=\left[b_{1}, \ldots, b_{s}\right], k=r-2 j$ e $i \geq 0$ determinado pelas condições:

$$
\begin{aligned}
\nu(A)+\left(b_{1}-1\right)+\cdots+\left(b_{i}-1\right) & \leq d-1 \\
\nu(A)+\left(b_{1}-1\right)+\cdots+\left(b_{i+1}-1\right) & >d-1 .
\end{aligned}
$$

Logo, existe um inteiro positivo $f$ tal que $f-1<b_{i+1}-1$ e

$$
\nu(A)+\left(b_{1}-1\right)+\cdots+\left(b_{i}-1\right)+(f-1)=d-1
$$

Seja $B^{\prime}=\left[b_{1}, \ldots, b_{i}, f, 1, \ldots, 1\right]$ a partição induzida de $d$, e $B^{\prime \prime}=\left[b_{i+1}-f+1, b_{i+2}, \ldots, b_{s}\right]$. Olhando para $B^{\prime \prime}$ como uma partição de $n$ símbolos $\left\{u_{1}, \ldots, u_{n}\right\}$ um dos quais pertence ao $f$-ciclo de $\beta^{\prime} \in B^{\prime}$ e os outros são símbolos distintos dos que têm $\beta^{\prime}$ nos seus ciclos de comprimento maior que 1. Pela escolha, $\nu\left(B^{\prime \prime}\right)=r$ e pela proposição $3.2 .1, B^{\prime \prime}$ pode 
realizar-se como produto de um $n$-ciclo e um $n-r+2 j$-ciclo nos símbolos $\left\{u_{1}, \ldots, u_{n}\right\}$. Equivalentemente existe $\beta^{\prime \prime} \in B^{\prime \prime}$ e um $n$-ciclo $\gamma \in S_{n}$ tal que $\gamma \beta^{\prime \prime} \in[(n-r+2 j), 1, \ldots, 1]$. Seja $\alpha \in A$, podemos assumir por 3.4 que $\alpha \beta^{\prime}$ é um $d$-ciclo. Tomemos

$$
\alpha \beta^{\prime}=\left(u_{1} \ldots w_{1} u_{2} \ldots w_{2} \ldots u_{n} \ldots w_{n}\right)
$$

e $\gamma=\left(u_{1} \ldots u_{n}\right)$. Seja $\beta=\beta^{\prime} \beta^{\prime \prime}$. O produto $\alpha \beta=\left(\alpha \beta^{\prime}\right) \beta^{\prime \prime}$ pode-se descrever como o obtido a partir de $\left(u_{1} \ldots u_{n}\right) \beta^{\prime \prime} \in[(n-r+2 j), 1, \ldots, 1]$ trocando cada $u_{j}$ pelo bloco $u_{j}, \ldots, w_{j}$ dado em $\alpha \beta^{\prime}$. Em outras palavras, $\alpha \beta$ é o produto de $r-2 j+1$ ciclos disjuntos tais que

$$
\nu(\alpha \beta)=d-(r-2 j+1)=(d-1)-r+2 j=(d-1)-k
$$

Corolario 3.2.7 Sejam $A, B \in \pi(d) \operatorname{com} \nu(A)+\nu(B) \geq d-1$ e $\nu(A)+\nu(B) \equiv d+$ $1(\bmod 2)$. Então existem $\alpha \in A, \beta \in B$ tais que $\langle\alpha, \beta\rangle$ age transitivamente sobre $\{1, \ldots, d\}$, sendo $\alpha \beta$ um d-ciclo.

Demonstração: Se $\nu(A)+\nu(B)=d-1$ é só olhar a proposição 3.2.5. Se $\nu(A)+\nu(B) \geq$ $d+1$ pegando $k=0$ na proposição 3.2 .6 temos que existem $\alpha, \beta$ tais que $\langle\alpha, \beta\rangle$ age transitivamente sobre $\{1, \ldots, d\}$ e $\nu(\alpha \beta)=d-1$ o que implica que $\alpha \beta$ seja um $d$-ciclo.

Proposição 3.2.8 Sejam $A, B \in \pi(d) \operatorname{com} \nu(A)+\nu(B)=d+2 q, q \geq 0$. Então existem $\alpha \in A, \beta \in B$ tais que $\langle\alpha, \beta\rangle$ age transitivamente sobre $\{1, \ldots, d\}$ e se $A=B=[2, \ldots, 2]$ então $\alpha \beta \in[d / 2, d / 2]$. Caso contrário, $\alpha \beta \in[d-1,1]$.

Demonstração: Se $A=B=[2, \ldots, 2] \operatorname{com} d=2 r$ e

$$
\begin{aligned}
& \alpha=\left(\begin{array}{ll}
1 & 2
\end{array}\right)(3 \quad 4) \ldots(2 r-12 r) \\
& \beta=\left(\begin{array}{ll}
2 & 3
\end{array}\right)(4 \quad 5) \ldots(2 r-2 \quad 2 r-1)(2 r \quad 1)
\end{aligned}
$$

então

$$
\alpha \beta=(1 \quad 3 \ldots(2 r-1))(2 r \quad 2 r-2 \ldots 2) \in[r, r] .
$$


Suponha que alguma das duas $A$ ou $B$ não está em $[2, \ldots, 2], \log$ o $A$ ou $B$ contém um termo maior que 2 , pois do contrário teríamos $\nu(A)+\nu(B)<r+r=2 r=d$, o que é absurdo. Sem perda de generalidade, podemos assumir que $A$ tem um dos seus termos maior que 2.

Seja $\alpha=\left(1 \ldots d_{1}\right)\left(d_{1}+1 \ldots d_{2}\right) \ldots\left(d_{r-1}+1 \ldots d_{r}\right) \in A$ com $d_{r}=d$ e $d_{1}>2$. Já que $\nu(A) \leq d-1, \nu(B)>0$. Seja. $B=\left[b_{1}, \ldots, b_{s}\right] \operatorname{com} b_{1} \geq 2$.

Considere as partições

$$
\begin{aligned}
& A^{\prime}=\left[a_{1}-1, a_{2}, \ldots, a_{r}\right] \\
& B^{\prime}=\left[b_{1}-1, b_{2}, \ldots, b_{s}\right]
\end{aligned}
$$

$a_{i+1}=d_{i+1}-d_{i}$ e $d_{0}=0$. Realizemos $A^{\prime} \operatorname{com} \alpha^{\prime}=\left(\begin{array}{lll}1 & 2 \ldots d_{1}-1\end{array}\right)\left(d_{1}+1 \ldots d_{2}\right) \ldots\left(d_{r-1}+\right.$ $\left.1 \ldots d_{r}\right)$. Estamos considerando $A^{\prime}$ e $B^{\prime}$ como partições de $\{1, \ldots, d\}-\left\{d_{1}\right\}$. Note que

$$
\begin{aligned}
\nu\left(A^{\prime}\right)+\nu\left(B^{\prime}\right) & =(\nu(A)-1)+(\nu(B)-1) \\
& =\nu(A)+\nu(B)-2 \\
& =d+2 q-2 \\
& =(d-1)+(2 q-1) .
\end{aligned}
$$

Pela proposição 3.2 .6 (proposição 3.2 .5 se $q=0$ ), podemos escolher $\alpha^{\prime}, \beta^{\prime}$ tais que $\left\langle\alpha^{\prime}, \beta^{\prime}\right\rangle$ age transitivamente sobre $\{1, \ldots, d\}-\left\{d_{1}\right\}$ e tal que $\nu\left(\alpha^{\prime} \beta^{\prime}\right)=(d-1)-1$, isto é, $\alpha^{\prime} \beta^{\prime}$ é um $(d-1)$-ciclo, onde $\beta^{\prime}$ pode-se escolher começando como $\left(b_{1}-1\right)$-ciclo $\left(1 \quad d_{1}+1 \ldots d_{b_{1}-2}+1\right)$. Seja $\alpha=\alpha^{\prime}\left(1 d_{1}\right)$ e $\beta=\left(1 d_{1}\right) \beta^{\prime}$, então $\alpha \beta=\alpha^{\prime} \beta^{\prime}$ é um $(d-1)$-ciclo. Mais ainda, $\langle\alpha, \beta\rangle$ é transitivo sobre $\{1, \ldots, d\}$ já que $\alpha$ move $d_{1}$ e $\alpha \beta$ é um $(d-1)$-ciclo nos símbolos diferentes de $d_{1}$. 


\subsection{Construindo uma Operação de Colagem sobre Ho- momorfismos no Grupo Simétrico.}

Seja $N$ uma superfície e $\left(M_{1}, \phi_{1}, N, B_{\phi_{1}}\right),\left(M_{2}, \phi_{2}, N, B_{\phi_{2}}\right)$ revestimentos ramificados conexos sobre $N$ de graus $n, m$ respectivamente que induzem as representações de Hurwitz:

$$
\Psi_{1}: \pi_{1}\left(\widehat{N}, z^{*}\right) \longrightarrow S_{n}, \Psi_{2}: \pi_{1}\left(\widehat{N}, z^{*}\right) \longrightarrow S_{m}
$$

É possível construir a soma direta delas e obter o homomorfismo

$$
\Psi_{1} \times \Psi_{2}: \pi_{1}\left(\widehat{N}, z^{*}\right) \longrightarrow S_{n+m}
$$

Mas esta construção não tem interesse geométrico pois o revestimento associado a ela não é conexo.

Em [BGKZ1] define-se uma operação de colagem entre $M_{1}$ e $M_{2}$ sendo que são superfícies de revestimento de $N$ para revestimentos ramificados específicos. ${ }^{2}$ Esta definição exige pegar sobre $M_{1}, M_{2}$ uma curva fechada simples que não reverte orientação e que não separa ${ }^{3}$, tal que cada uma destas curvas é enviada por $\phi_{1}, \phi_{2}$ na mesma potência de um laço em $N$.

Nesta seção, vamos descrever a versão algébrica da operação de colagem mas só sobre revestimentos ramificados do toro ou da garrafa de Klein, onde a ramificação está localizada em um único ponto.

Para facilitar a notação, sejam

$$
\begin{aligned}
& G_{+}=\left\langle a, b, c \mid[a, b] c^{-1}=1\right\rangle \\
& G_{-}=\left\langle a, b, c \mid a b a b^{-1} c^{-1}=1\right\rangle
\end{aligned}
$$

os grupos fundamentais do toro e da garrafa de Klein depois de tirar um pequeno disco respectivamente. Assim, quando falamos do grupo $G$ estamos fazendo referencia a $G_{+}$ou G- separadamente.

\footnotetext{
${ }^{2}$ Ver [BGKZ1], seção 2.3 página 6 .

${ }^{3}$ Em geral a propriedade de não separabilidade define-se assim: Seja $X$ um espaço topológico e $A C X$, dizemos que $A$ não separa $X$, se para todo aberto $U$ de $X, U-A$ é conexo.
} 
Sejam

$$
\begin{aligned}
& \Psi_{1}: G \longrightarrow S_{n} \\
& \Psi_{2}: G \longrightarrow S_{m}
\end{aligned}
$$

representações associadas a revestimentos ramificados conexos cuja ramificação está localizada em um único ponto da base, o mesmo para ambos.

Para todo $g \in G$ vamos denotar por $\widehat{g}_{i}$ a permutaçăo $\Psi_{i}(g), i=1,2$. O subgrupo de permutações gerado por $\widehat{a}_{1}, \widehat{b}_{1}$ age transitivamente sobre $\{1, \ldots, n\}$, assim como o subgrupo gerado por $\widehat{a}_{2}, \widehat{b}_{2}$ sobre $\{n+1, \ldots, n+m\}$, pela conexidade dos revestimentos.

Vamos assumir, que existe um laço em $M_{1}$ e um laço em $M_{2}$ tais que são levados homeomorficamente no mesmo laço na classe de homotopia de $a \in G$. Portanto existe um símbolo $i_{1}$ em $\{1, \ldots, n\}$ e um símbolo $i_{2}$ em $\{n+1, \ldots, n+m\}$ que ficam fixos por $\widehat{a}_{1} \mathrm{e}$ $\widehat{a}_{2}$ respectivamente. Se $n \geq 2$, pela transitividade, nem $\widehat{b}_{1}$ fixa $i_{1}$, nem $\widehat{b}_{2}$ fixa $i_{2}$.

Seja $\widehat{a}=\widehat{a}_{1} \times \widehat{a}_{2} \in S_{n+m}$, a soma direta de $\widehat{a}_{1}$ e $\widehat{a}_{2}$. Assim, $\widehat{a}$ age sobre $\{1, \ldots, n\}$ como $\widehat{a}_{1}$ e sobre $\{n+1, \ldots, n+m\}$ como $\widehat{a}_{2}$.

Por outro lado, definimos $\widehat{b} \in S_{n+m}$ como a soma direta de $\widehat{b}_{1}$ e $\widehat{b}_{2}$, vezes a transposição $\left(i_{1} i_{2}\right)$, isto é, $\widehat{b}=\left(\widehat{b}_{1} \times \widehat{b}_{2}\right)\left(i_{1} i_{2}\right)$.

Vamos mostrar que $\widehat{c}=\widehat{c}_{1} \times \widehat{c}_{2}$ é a permutação que corresponde a $c=[a, b]$ ou $c=a b a b^{-1}$ dependendo do caso (proposição 3.3.2).

O resultado da operação colagem sobre as representações $\Psi_{1}$ e $\Psi_{2}$ é a representação

$$
\begin{aligned}
\Psi: G & \longrightarrow S_{n+m} \\
a & \longmapsto \widehat{a} \\
b & \longmapsto \widehat{b} .
\end{aligned}
$$

Proposição 3.3.1 Se os subgrupos $\left\langle\widehat{a_{1}}, \widehat{b_{1}}\right\rangle,\left\langle\widehat{a_{2}}, \widehat{b_{2}}\right\rangle$ agem transitivamente sobre os conjuntos $\{1, \ldots, n\} \in\{n+1, \ldots, n+m\}$ respectivamente entăo o subgrupo $\langle\widehat{a}, \widehat{b}\rangle$ age transitivamente sobre $\{1, \ldots, n+m\}$.

Demonstração: Observemos que a órbita de qualquer elemento $i \leq n$ contem $i_{2}$. De fato, pela transitividade, existe uma palavra $w$ nas permutações $\widehat{a_{1}}$, $\widehat{b_{1}}$ que leva $i$ no 
símbolo $i_{1} \cdot{\widehat{b_{1}}}^{-1}$. Vamos pegar a menor destas palavras e notemos que se trocamos $\widehat{a_{1}}$ por $\widehat{a}$ e $\widehat{b_{1}}$ por $\widehat{b}$, a palavra $w^{\prime}$ obtida em $\widehat{a}$ e $\widehat{b}$ também leva o símbolo $i$ no $i_{1} \cdot \widehat{b}_{1}^{-1}$. Agora

$$
\begin{aligned}
\left(i . w^{\prime}\right) \hat{b} & =i_{1} \cdot\left(\widehat{b}_{1}^{-1} \widehat{b}\right) \\
& =i_{1} \cdot\left(\widehat{b}_{1}^{-1}\left(\widehat{b_{1}} \times \widehat{b_{2}}\right)\left(i_{1} i_{2}\right)\right) \\
& =i_{1} \cdot\left(i_{1} \quad i_{2}\right) \\
& =i_{2}
\end{aligned}
$$

logo existe uma palavra nas permutaçōes $\widehat{a}, \widehat{b}$ que leva o $i$ no $i_{2}$. Analogamente existe uma palavra em $\widehat{a}, \widehat{b}$ que leva os $j \geq n+1$ no símbolo $i_{1}$, em particular existe uma palavra em $\widehat{a}, \widehat{b}$ que leva $i_{2}$ em $i_{1}$. Portanto é possível enviar todos os elementos em $i_{1}$ e assim a ação é transitiva.

No estudo dos revestimentos ramificados sobre superfícies não orientáveis o quasicomutador $[a, b]_{-}=a b a b^{-1}$ tem um papel importante. Para unificar a notação, vamos generalizar a noção de comutador. Sejam $r, s \in \mathbb{Z}$, definimos

$$
[a, b]_{r, s}=a^{r} b a^{s} b^{-1}
$$

Assim, $[a, b]_{1,-1}=[a, b]$ e $[a, b]_{1,1}=[a, b]_{-}$.

Proposição 3.3.2 Para qualquer par de inteiros $r, s$,

$$
[\widehat{a}, \widehat{b}]_{r, s}=\left[\widehat{a_{1}}, \widehat{b_{1}}\right]_{r, s} \times\left[\widehat{a_{2}}, \widehat{b_{2}}\right]_{r, s}
$$

Demonstração: Seja $i \leq n$ e consideremos $\widehat{a_{1}}=\widehat{a_{1}} \times i d, \widehat{b_{1}}=\widehat{b_{1}} \times i d, \widehat{a_{2}}=i d \times \widehat{a_{2}}, \widehat{b_{2}}=$ id $\times \widehat{b_{2}}$ em $S_{n+m}$. Então

$$
\begin{aligned}
i . \widehat{a}^{r} \widehat{b} \widehat{a}^{s} \widehat{b}^{-1} & =i \cdot\left(\widehat{a_{1}} \times \widehat{a_{2}}\right)^{r}\left[\left(\widehat{b_{1}} \times \widehat{b_{2}}\right)\left(i_{1} i_{2}\right)\right]\left(\widehat{a_{1}} \times \widehat{a_{2}}\right)^{s}\left[\left(\widehat{b_{1}} \times \widehat{b_{2}}\right)\left(i_{1} i_{2}\right)\right]^{-1} \\
& =i . \widehat{a}_{1}^{r} \widehat{b_{1}}\left(i_{1} i_{2}\right) \widehat{a}^{s} \widehat{b}^{-1}
\end{aligned}
$$

Suponhamos que $i . \widehat{a}_{1}^{r} \widehat{b_{1}} \neq i_{1}$, assim $i . \widehat{a}^{r} \widehat{b}^{3} \widehat{a}^{-1}=i \cdot \widehat{a}_{1}^{r} \widehat{b}_{1} \widehat{a}_{1}^{s} \widehat{b}^{-1}$. Já que $\widehat{a_{1}}$ fixa $i_{1}$, temos que $i . \widehat{a}_{1}^{r}{\widehat{b_{1}}}^{a_{1}} \neq i_{1}$. Daqui, sob a ação de $\widehat{b}^{-1}$ a transposição $\left(i_{1} i_{2}\right)$ não se aplica, portanto 
$i \cdot \widehat{a}^{r} \widehat{b} \widehat{a}^{s} \widehat{b}^{-1}=i \cdot \widehat{a}_{1}^{r} \widehat{b}_{1} \widehat{a}_{1}^{s} \widehat{b}_{1}^{-1}$. Agora se $i \cdot \widehat{a}_{1}^{r} \widehat{b_{1}}=i_{1}$ então:

$$
\begin{aligned}
i . \widehat{a}^{r} \widehat{b} \widehat{a}^{s} \widehat{b}^{-1} & =i_{1} \cdot\left(i_{1} i_{2}\right) \widehat{a}^{s} \widehat{b}^{-1} \\
& =i_{2} \cdot \widehat{a}^{s} \widehat{b}^{-1} \\
& =i_{2} \cdot \widehat{b}^{-1} \\
& =i_{1} \cdot \widehat{b}_{1}^{-1} \\
& =i_{1} \cdot \widehat{a}_{1}^{s} \widehat{b}_{1}^{-1} \\
& =i \cdot \widehat{a}_{1}^{r} \widehat{b}_{1} \widehat{a}_{1}^{s}{\widehat{b_{1}}}^{-1}
\end{aligned}
$$

Para $i \geq n+1$ procede-se analogamente.

Teorema 3.3.3 Para cada partição $A=\left[d_{1}, \ldots, d_{r}\right]$ do número d com defeito positivo par, $\nu(A)=\left(d_{1}-1\right)+\cdots+\left(d_{r}-1\right)=d-r>0$, existem permutações $\widehat{a}, \widehat{b} \in S_{d}$ com as seguintes propriedades:

(1) O subgrupo de $S_{d}$ gerado por $\widehat{a}, \widehat{b}$ age transitivamente sobre $\{1, \ldots, d\}$.

(2) O comutador $[\widehat{a}, \widehat{b}]$ consiste de ciclos de comprimentos $d_{1}, \ldots, d_{r}$.

(3) O simbolo 1 fica fixo por $\widehat{a}$.

(4) O simbolo 1 fica fixo por $\widehat{b}[\widehat{a}, \widehat{b}]$ ou por $\widehat{b}\left[\widehat{a}^{2}, \widehat{b}\right]$.

Demonstração: A prova é por indução sobre $r$. Assumamos $d_{1} \geq \cdots \geq d_{r}$.

Se $r=1$, então $A=[d]$ e pela hipóteses $\nu(A)=d-1$ é par e maior que zero, logo $d$ é ímpar e maior que 2 , donde $d=2 k+1 \operatorname{com} k>0$. Sejam

$$
\begin{gathered}
\widehat{a}=\left(\begin{array}{ccccccc}
1 & \ldots & k & k+1 & \ldots & 2 k & 2 k+1 \\
1 & \ldots & k & k+2 & \ldots & 2 k+1 & k+1
\end{array}\right) \\
\widehat{b}=\left(\begin{array}{ccccccc}
1 & \ldots & k & k+1 & k+2 & \ldots & 2 k+1 \\
2 k+1 & \ldots & k+2 & k+1 & 1 & \ldots & k
\end{array}\right) .
\end{gathered}
$$

Vamos conferir que $\widehat{a}, \hat{b}$ satisfazem as propriedades (1)-(4):

(2): No nosso caso $[\widehat{a}, \widehat{b}]$ tem que ser um $d$-ciclo. Pelas definições de $\widehat{a}, \widehat{b}$ :

$$
[\widehat{a}, \widehat{b}]=\left(\begin{array}{ccccccc}
1 & \ldots & k & k+1 & \ldots & 2 k & 2 k+1 \\
2 & \ldots & k+1 & k+2 & \ldots & 2 k+1 & 1
\end{array}\right)
$$


é de fato um $d$-ciclo.

(1): É imediato de (2).

(3): É claro da definição de $\widehat{a}$.

(4): $1 . \widehat{b}[\widehat{a}, \widehat{b}]=(2 k+1)[\hat{a}, \widehat{b}]=1$.

Para $r \geq 2$. Se existe um $d_{j}$ impar, consideramos $A^{\prime}=\left[d_{1}, \ldots, d_{j-1}, d_{j+1}, \ldots, d_{r}\right] \mathrm{e}$ $A^{\prime \prime}=\left[d_{j}\right]$, partições dos números $d^{\prime}=d-d_{j}, d^{\prime \prime}=d_{j}$ respectivamente. Se $d_{r}=1$, fazemos $j=r$. Já que $\nu\left(\left[d_{j}\right]\right)=d_{j}-1$ é par e $\nu(A)=\nu\left(A^{\prime}\right)+\nu\left(A^{\prime \prime}\right)$ então $\nu\left(A^{\prime}\right)$ também é par. Mais ainda ambos $\nu\left(A^{\prime}\right), \nu\left(A^{\prime \prime}\right)$ são maiores que zero e vale a hipóteses de indução, exceto se $d^{\prime \prime}=d_{j}=1$. Mas para este caso as propriedades (1)-(4) são satisfeitas trivialmente. Portanto, existem permutações $\widehat{a}_{1}, \widehat{b}_{1}$ e $\widehat{a}_{2}, \widehat{b}_{2}$ tais que $\left[\widehat{a}_{1}, \widehat{b}_{1}\right] \in A^{\prime}$ e $\left[\widehat{a}_{2}, \widehat{b}_{2}\right] \in A^{\prime \prime}$. Além disso, já que estes pares de permutações satisfazem a propriedade (3) do teorema, podemos aplicar a operação de colagem entre elas e assim obtemos as permutações $\widehat{a}, \widehat{b}$ que satisfazem as propriedades (1)-(4). Vejamos:

(1): Da proposição 3.3.1.

(2): Da proposição 3.3.2.

(3): $1 . \widehat{a}=1 . \widehat{a}_{1}=1$.

(4): Na operação de colagem usemos $i_{1}=1$. Temos $1 . \hat{b}=1 . \hat{b}_{1}\left(1 i_{2}\right)=1 . \hat{b}_{1}$ e, usando a proposição 3.3 .2 ,

$$
\begin{aligned}
1 . \widehat{b}[\widehat{a}, \widehat{b}] & =1 \cdot \widehat{b}_{1}[\widehat{a}, \widehat{b}] \\
& =1 \cdot \widehat{b}_{1}\left(\left[\widehat{a}_{1}, \widehat{b}_{1}\right] \times\left[\widehat{a}_{2}, \widehat{b}_{2}\right]\right) \\
& =1 \cdot \widehat{b}_{1}\left[\widehat{a}_{1}, \widehat{b}_{1}\right] \\
& =1
\end{aligned}
$$

ou

$$
\begin{aligned}
1 . \widehat{b}\left[\widehat{a}^{2}, \widehat{b}\right] & =1 . \widehat{b}_{1}\left(\left[\widehat{a}_{1}^{2}, \widehat{b}_{1}\right] \times\left[\widehat{a}_{2}^{2}, \widehat{b}_{2}\right]\right) \\
& =1 . \widehat{b}_{1}\left[\widehat{a}_{1}^{2}, \widehat{b}_{1}\right] \\
& =1 .
\end{aligned}
$$

Agora, se todos os $d_{i}$ são pares, então $d=2 k, k \geq 2$, e já que $\nu(A)=d-r$ é par, segue que $r$ é par. 
Se $r=2$, então $A=\left[d_{1}, d_{2}\right] . A \operatorname{ssim} l=\left(d_{1}-d_{2}\right) / 2$ é um inteiro entre zero e $k-2$. Seja $d_{1}=k+l$ e $d_{2}=k-l$.

Consideremos uma situação mais geral assumindo só que $d_{2}>1$, isto é $l \leq k-2$. Em outras palavras, no que segue não precisamos de $d_{1}, d_{2}$ pares, só que $d_{2}>1$. Veja, para. $0 \leq l \leq k-2$ definimos

$$
\begin{aligned}
& \widehat{a}=\left(\begin{array}{cccccccc}
1 & \ldots & k-1 & k & k+1 & \ldots & 2 k-1 & 2 k \\
1 & \ldots & k-1 & k+1 & k+2 & \ldots & 2 k & k
\end{array}\right)
\end{aligned}
$$

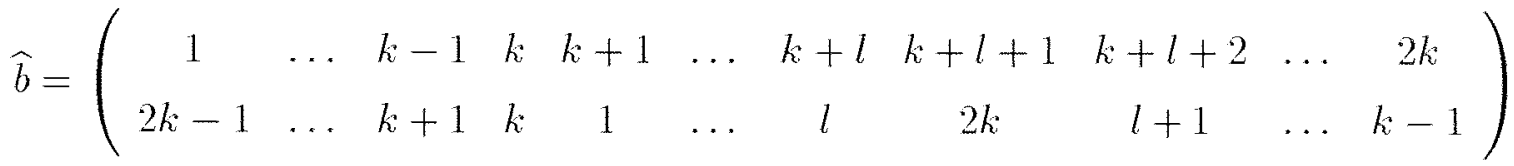

Se $l=0$ ignoramos desde a coluna $k+1$ até a coluna $k+l$. Confiramos (1)-(4):

$$
[\widehat{a}, \widehat{b}]=\left(\begin{array}{cccccccc}
1 & \ldots & k+l-1 & k+l & k+l+1 & \ldots & 2 k-1 & 2 k \\
2 & \ldots & k+l & 1 & k+l+2 & \ldots & 2 k & k+l+1
\end{array}\right)
$$

donde $[\widehat{a}, \widehat{b}]=(12 \ldots k+l)(k+l+1 \ldots 2 k)$ é produto de ciclos de comprimentos $d_{1}$ e $d_{2}$ respectivamente.

(1): Está garantida por $\widehat{a}$ pois leva $2 k \mathrm{em} k$.

(3): Da definição de $\widehat{a}$.

(4): $1 . \widehat{b}\left[\widehat{a}^{2}, \widehat{b}\right]=(2 k-1) \cdot\left[\widehat{a}^{2}, \widehat{b}\right]=1$.

Se $r \geq 3$ então $r \geq 4$ e $d_{r} \geq 2$, pois $r$ e $d_{r}$ são pares. Podemos aplicar a operação de colagem a dois pares de permutaçōes tais que seus comutadores sejam do tipo $A^{\prime}=$ $\left[d_{1}, \ldots, d_{r-2}\right]$ e $A^{\prime \prime}=\left[d_{r-1}, d_{r}\right]$, sendo $A^{\prime}$ e $A^{\prime \prime}$ partições dos números $d^{\prime}=d-d_{r-1}-d_{r}$ e $d^{\prime \prime}=d_{r-1}+d_{r}$ respectivamente.

Corolario 3.3.4 Seja $\widehat{c} \in S_{d}$ uma permutação par não trivial. Então existem permutaçöes $\widehat{a}, \widehat{b}$ com as seguintes propriedades:

(1) $\langle\widehat{a}, \widehat{b}\rangle$ age transitivamente sobre $\{1, \ldots, d\}$.

(2) $\widehat{c}=[\widehat{a}, \widehat{b}]$.

(3) a fixa 1 .

(4) O 1 fica fixo por $\widehat{b}[\widehat{a}, \widehat{b}]$ ou por $\widehat{b}\left[\widehat{a}^{2}, \widehat{b}\right]$. 
Teorema 3.3.5 Para cada partição $A=\left[d_{1}, \ldots, d_{r}\right]$ do número d com defeito positivo par, $\nu(A)=\left(d_{1}-1\right)+\cdots+\left(d_{r}-1\right)=d-r>0$, existem permutações $\widehat{a}, \widehat{b} \in S_{d}$ e um inteiro positivo q com as seguintes propriedades:

(1) O subgrupo de $S_{d}$ gerado por $\widehat{a}, \widehat{b}$ age transitivamente sobre $\{1, \ldots, d\}$.

(2) O quasicomutador $[\widehat{a}, \widehat{b}]_{-}$consiste de ciclos de comprimentos $d_{1}, \ldots, d_{r}$.

(3) O simbolo 1 fica fixo por $\widehat{a}$.

(4) O simbolo 1 fica fixo por $\widehat{b}[\widehat{a}, \widehat{b}]_{-}^{q}$.

Demonstração: A prova é por indução sobre $r$. Assumamos $d_{1} \geq \cdots \geq d_{r}$.

Se $r=1, A=[d]$ e já que $\nu(A)=d-1$ é par, então $d=2 k+1, k \geq 1$. Definamos

$$
\begin{gathered}
\widehat{a}=\left(\begin{array}{ccccccc}
1 & \ldots & k & k+1 & \ldots & 2 k & 2 k+1 \\
1 & \ldots & k & k+2 & \ldots & 2 k+1 & k+1
\end{array}\right) \\
\widehat{b}=\left(\begin{array}{ccccccc}
1 & 2 & \ldots & k+1 & k+2 & \ldots & 2 k+1 \\
2 k+1 & k+1 & \ldots & 2 k & 1 & \ldots & k
\end{array}\right)
\end{gathered}
$$

vejamos que satisfazem (1)-(4):

$(\mathfrak{Q )}$ :

$$
[\widehat{a}, \widehat{b}]_{-}=\left(\begin{array}{ccccccc}
1 & \ldots & k & k+1 & \ldots & 2 k & 2 k+1 \\
2 & \ldots & k+1 & k+2 & \ldots & 2 k+1 & 1
\end{array}\right)
$$

é de fato um $d$-ciclo.

(1): E imediato de (2).

(3): Da definição de $\widehat{a}$.

(4): $\widehat{b}[\widehat{a}, \hat{b}]_{-}^{q}$ fixa 1 para $q=1$.

Para $r \geq 2$. Se existe um $d_{j}$ ímpar, usamos os mesmos argumentos da prova do teorema 3.3.3. É por isto que vamos assumir todos os $d_{j}$ pares, portanto em $\nu(A)=d-r$, $d$ e $r$ são pares.

Se $r=2$, então $A=\left[d_{1}, d_{2}\right]$. Seja $l=\left(d_{1}-d_{2}\right) / 2$ assim $d_{1}=k+l$ e $d_{2}=k-l$. Vamos obter conclusões mais gerais se assumimos só que $d_{2}>1$, isto é, $l \leq k-2$. 
Para $0 \leq l \leq k-2$ definimos

$$
\begin{aligned}
& \widehat{a}=\left(\begin{array}{cccccccc}
1 & \ldots & k-1 & k & k+1 & \ldots & 2 k-1 & 2 k \\
1 & \ldots & k-1 & k+1 & k+2 & \ldots & 2 k & k
\end{array}\right) \\
& \widehat{b}=\left(\begin{array}{cccccccccc}
1 & \ldots & k & k+1 & \ldots & k+l & k+l+1 & k+l+2 & \ldots & 2 k \\
k & \ldots & 2 k-1 & 1 & \ldots & l & 2 k & l+1 & \ldots & k-1
\end{array}\right)
\end{aligned}
$$

Se $l=0$ ignoramos desde a coluna $k+1$ até a coluna $k+l$. Verifiquemos (1)-(4):

$(2)$ : Facilmente verifica-se que $[\widehat{a}, \widehat{b}]_{-}=(12 \ldots k-1 \ldots k+l)(k+l+1 \ldots 2 k)$.

(1): $\widehat{a}$ leva $2 k \mathrm{em} k$ e isto garante a transitividade.

(3): Da definição de $\widehat{a}$.

(4): $\widehat{b}$ leva o 1 no $k$, e $[\widehat{a}, \widehat{b}]_{-}^{l+1}$ leva $k$ no 1 .

Para os casos $r \geq 3$, isto é $r \geq 4$, usamos os mesmos argumentos da prova do teorema 3.3 .3 .

Corolario 3.3.6 Seja $\widehat{c} \in S_{d}$ uma permutaçäo par não trivial. Então existem permutaçôs $\widehat{a}, \widehat{b}$ e um inteiro positivo q com as seguintes propriedades:

(1) $\langle\widehat{a}, \widehat{b}\rangle$ age transitivamente sobre $\{1, \ldots, d\}$.

(2) $\widehat{c}=[\widehat{a}, \widehat{b}]_{-}$.

(3) $\widehat{a}$ fixa 1 .

(4) $\widehat{b}[\widehat{a}, \widehat{b}]_{-}^{q}$ fixa 1 .

Notemos que $[\widehat{a}, \widehat{b}]_{-}=\widehat{g}^{2} \widehat{b}^{-2}$, onde $\widehat{g}=\widehat{a} \widehat{b}$. Assim, $\widehat{c}$ pode escrever-se como produto de quadrados de duas permutações que geram um subgrupo de $S_{d}$ que age transitivamente sobre $\{1, \ldots, d\}$. 


\section{Capítulo 4}

\section{Revestimentos Ramificados sobre}

\section{Superfícies Fechadas}

Neste capítulo apresentamos a solução da Questão 1 quando $N$ é fechada e diferente da esfera, baseados nos artigos [Hu], [EKS] e [Ez].

Seja $\left(M, \phi, N, B_{\phi}\right), B_{\phi}=\left\{x_{1}, \ldots, x_{k}\right\}$, um revestimento ramificado de grau $d$ finito, onde $N$ é uma superfície fechada. Segundo as possíveis presentações do grupo fundamental de $\widehat{N}=N-B_{\phi}$ da página 15 , a existência de uma representação de Hurwitz $\Psi$, implica que

$$
\Psi\left(\left\{v_{x_{1}}^{*}\right\} \ldots\left\{v_{x_{k}}^{*}\right\}\right)= \begin{cases}1 & N=\mathbb{S}^{2} \\ {\left[\Psi\left(\gamma_{n}\right), \Psi\left(\beta_{n}\right)\right] \cdot\left[\Psi\left(\gamma_{n-1}\right), \Psi\left(\beta_{n-1}\right)\right] \ldots\left[\Psi\left(\gamma_{1}\right), \Psi\left(\beta_{1}\right)\right]} & N=\mathbb{T}_{n} \\ \Psi\left(\beta_{n}\right)^{2} \cdot \Psi\left(\beta_{n-1}\right)^{2} \ldots \Psi\left(\beta_{1}\right)^{2} & N=\mathbb{R} \mathbb{P}_{n}\end{cases}
$$

Observemos que sempre $\Psi\left(\left\{v_{x_{1}}^{*}\right\} \ldots\left\{v_{x_{k}}^{*}\right\}\right)=\sigma_{1} \ldots \sigma_{k}$ é uma permutação par. Isto quer dizer que se $A_{i}=\left[a_{i_{1}}, \ldots, a_{i_{i}}\right], j_{i}=\#\left(\phi^{-1}\left(x_{i}\right)\right)$, é a partição associada a $\sigma_{i}$, segundo os resultados da seção 3.1 do capítulo anterior, então

$$
\nu(\mathscr{D})=\sum_{i=1}^{k} \nu\left(A_{i}\right) \equiv 0 \quad(\bmod 2)
$$

Se $\mathscr{L}$ é um conjunto de partições não triviais de $d$ que satisfaz (4.1), dizemos que $\mathscr{D}$ é um dado de ramificação virtual. 
Proposição 4.1 Seja $N$ uma superficie fechada $e \mathscr{D}=\left\{A_{1}, \ldots, A_{k}\right\}$ um dado de ramificação virtual. Se $\chi(N) \leq 0, \mathscr{D}$ é relizável. Mais que isso, se $N$ é não orientável $e d$ é par, a superficie de revestimento pode ser escolhida não orientável.

Demonstração: Vamos mostrar que partindo da congruência (4.1) é possivel construir uma representação de Hurwitz $\Psi$. Basta definir o homomorfismo $\Psi: \pi_{1}\left(\widehat{N}, z^{*}\right) \longrightarrow S_{d}$ nos geradores de $\pi_{1}\left(\widehat{N}, z^{*}\right)$. Mas lembremos que:

$$
\pi_{1}\left(\widehat{N}, z^{*}\right)= \begin{cases}\left\langle\left\{v_{1}^{*}\right\}, \ldots,\left\{v_{k}^{*}\right\}, \beta_{1}, \gamma_{1}, \ldots, \beta_{n}, \gamma_{n} \mid\left\{v_{1}^{*}\right\} \ldots\left\{v_{k}^{*}\right\}\left[\beta_{1}, \gamma_{1}\right] \ldots\left[\beta_{n}, \gamma_{n}\right]=1\right\rangle & N=\mathbb{T}_{n} \\ \left\langle\left\{v_{1}^{*}\right\}, \ldots,\left\{v_{k}^{*}\right\}, \beta_{1}, \ldots, \beta_{n} \mid\left\{v_{1}^{*}\right\} \ldots\left\{v_{k}^{*}\right\} \beta_{1}^{2} \ldots \beta_{n}^{2}=1\right\rangle & N=\mathbb{R}_{n}\end{cases}
$$

Portanto, queremos que a relação nos grupos fundamentais acima tenha a identidade como imagem na definição de $\Psi$. Por outro lado, se $\nu(\mathscr{D}) \equiv 0(\bmod 2)$, então $\Psi\left(\left\{v_{1}^{*}\right\} \ldots\left\{v_{k}^{*}\right\}\right)$ tem que ser uma permutação par.

Se $N$ é orientável, pelo corolário 3.2 .2 podemos definir $\Psi$ tal que $\Psi\left(\left\{v_{1}^{*}\right\} \ldots\left\{v_{r}^{*}\right\}\right)=$ $\left[\Psi\left(\gamma_{1}\right), \Psi\left(\beta_{1}\right)\right]$ sendo $\Psi\left(\gamma_{1}\right)$ um $d$-ciclo e $\Psi\left(\beta_{i}\right)=\Psi\left(\gamma_{i}\right)=1$, para $1<i \leq n$. Donde $\Psi\left(\left\{v_{1}^{*}\right\} \ldots\left\{v_{k}^{*}\right\}\left[\beta_{1}, \gamma_{1}\right] \ldots\left[\beta_{n}, \gamma_{n}\right]\right)=1$. E já que $\Psi\left(\gamma_{1}\right)$ é um $d$-ciclo, isto garante a conexidade da superfície de revestimento.

Se $N$ é não orientável, analogamente, pelo corolário 3.2 .2 podemos definir $\Psi$ tal que $\Psi\left(\left\{v_{1}^{*}\right\} \ldots\left\{v_{r}^{*}\right\}\right)=\Psi\left(\beta_{2}\right)^{2} \cdot \Psi\left(\beta_{1}\right)^{2}$ sendo $\Psi\left(\beta_{2}\right) . \Psi\left(\beta_{1}\right)$ um $d$-ciclo e $\Psi\left(\beta_{j}\right)=1,2<j \leq$ $n$. Donde $\Psi\left(\left\{v_{1}^{*}\right\} \ldots\left\{v_{k}^{*}\right\} \beta_{1}^{2} \ldots \beta_{n}^{2}\right)=1$. E já que $\Psi\left(\beta_{2}, \beta_{1}\right)$ é um $d$-ciclo, a superfície de revestimento será conexa. Finalmente para demonstrar a última afirmação da proposição é importante lembrar que se $d$ é ímpar, a superfície de revestimento é não orientável (proposição 2.6). Se dé par, combinando a demonstração da afirmação 3 do corolário 3.2 .2 e a proposição 3.2 .3 podemos definir $\Psi\left(\beta_{2}\right)$ como sendo uma permutação que fixa um elemento. Daqui, pelo lema $2.5 M$ será não orientável.

O quê acontece quando $\chi(N)>0$ ?, isto é, o quê acontece quando $N$ é o plano projetivo ou a esfera?

Da teoria já estudada temos que se $\mathscr{D}$ é o dado de ramificação de $\left(M, \phi, N, B_{\phi}\right)$, necessariamente $\mathscr{T}$ satisfaz:

1. $\nu(\mathscr{D}) \equiv 0(\bmod 2)$ 
2. Pelo corolário 2.7:

- $\nu(\mathscr{D}) \geq d-1$, para $N=\mathbb{R} \mathrm{P}^{2}$ e $M$ não orientável.

- $\nu(\mathscr{D}) \geq d-2$, para $N=\mathbb{R} \mathrm{P}^{2}$ e $M$ orientável.

- $\nu(\mathscr{D}) \geq 2 d-2$, para $N=\mathbb{S}^{2}$

Chamaremos a combinação do primeiro enunciado com um dos itens do segundo as Condiçōes de Hurwitz.

Teorema 4.2 Seja $N$ o plano projetivo e $\mathscr{D}=\left\{A_{1}, \ldots, A_{k}\right\}$ um dado de ramificação virtual. $\mathscr{D}$ é realizável se e somente se $\mathscr{D}$ satisfaz as condiçós de Hurwitz $\nu(\mathscr{D}) \equiv 0$ $(\bmod 2)$ e $\nu(\mathscr{D}) \geq d-1$. Mais ainda, a superficie de revestimento pode ser escolhida näo orientável.

Demonstração: Resta garantir a existência de um revestimento ramificado $\left(M, \phi, N, B_{\phi}\right)$ que realize $\mathscr{D}$. Precisamos então definir uma representação

$$
\Psi:\left\langle\left\{v_{1}^{*}\right\} \ldots\left\{v_{k}^{*}\right\}, \beta_{1} \mid\left\{v_{1}^{*}\right\} \ldots\left\{v_{k}^{*}\right\} \beta_{1}^{2}=1\right\rangle \longrightarrow S_{d}
$$

tal que $\Psi\left(\left\{v_{i}^{*}\right\}\right)=\alpha_{i} \in A_{i}, 1 \leq i \leq k, \operatorname{e~} \Psi\left(\left\{v_{1}^{*}\right\} \ldots\left\{v_{k}^{*}\right\} \beta_{1}^{2}\right)=1$.

Seja $\nu(\mathscr{D})=2 s, s \in \mathbb{Z}^{+}$. Pode acontecer:

1. $d$ impar. Neste caso $\nu(\mathscr{D})=\sum_{i=1}^{k} \nu\left(A_{i}\right)=(d-1)+r$, com $d-1$ e $r \geq 0$ pares. $\operatorname{Logo} \nu\left(A_{1}\right)+\nu\left(A_{2}\right)=(d-1)+t, t=r-\sum_{i=3}^{k} \nu\left(A_{i}\right)$.

(a) Se $t>0$, pela proposição 3.2 .6 , para todo $R, 0 \leq R \leq t$ e $R \equiv t(\bmod 2)$ pode-se escolher $\alpha_{1} \in A_{1}, \alpha_{2} \in A_{2}$ tais que $\left\langle\alpha_{1}, \alpha_{2}\right\rangle$ age transitivamente sobre $\{1, \ldots, d\}$ e $\nu\left(\alpha_{1} \alpha_{2}\right)=(d-1)-R$.

(b) Se $t \leq 0$, pela proposição 3.2 .5 , existem $\alpha_{1} \in A_{1}$ e $\alpha_{2} \in A_{2}$ tais que o grupo $\left\langle\alpha_{1}, \alpha_{2}\right\rangle$ age sobre $\{1, \ldots, d\}$ determinando $t^{\prime}+1$ órbitas, $t^{\prime}=-t$, e se $C$ denota a partição de $d$ dada pelas as órbitas da ação de $\left\langle\alpha_{1}, \alpha_{2}\right\rangle$ sobre $\{1, \ldots, d\}$, então $\alpha_{1} \alpha_{2} \in C$. 
Seja $A_{12}=\left\|\alpha_{1} \alpha_{2}\right\|$, temos que $\nu\left(A_{12}\right)+\nu\left(A_{3}\right)=(d-1)+t_{1}, t_{1}=r-\sum_{i=4}^{k} \nu\left(A_{i}\right)$. Fazendo uma análise para $t_{1}$ análoga à feita anteriormente para $t$ vamos ter que $\left\langle\alpha_{1}, \alpha_{2}, \alpha_{3}\right\rangle$ age transitivamente sobre $\{1, \ldots, d\}$ ou age determinando $1-t_{1}$ órbitas dependendo se $t_{1}>0$ ou $t_{1} \leq 0$ respectivamente, e achamos um $A_{123}=\left\|\alpha_{1} \alpha_{2} \alpha_{3}\right\|$ tal que $\nu\left(A_{123}\right)+\nu\left(A_{4}\right)=(d-1)+t_{2}, t_{2}=r-\sum_{i=5}^{k} \nu\left(A_{i}\right)$. Agora analisamos $t_{2}$ e continuamos assim por diante até definir um $A_{12 \ldots(k-1)}=\left\|\alpha_{1} \ldots \alpha_{k-1}\right\|$ tal que $\nu\left(A_{1 \ldots(k-1)}\right)+\nu\left(A_{k}\right)=(d-1)+r, \operatorname{logo}$ pela proposição 3.2 .6 , existe $\alpha_{k} \in A_{k}$ tal que $\left\langle\alpha_{1} \ldots \alpha_{k-1}, \alpha_{k}\right\rangle$ age transitivamente sobre $\{1, \ldots, d\}$ e $\nu\left(\alpha_{1} \ldots \alpha_{k}\right)=d-1$ pois $r \equiv 0$ $(\bmod 2)$. Portanto $\alpha_{1} \ldots \alpha_{k}$ é um $d$-ciclo par e existe $\widehat{\beta}_{1} \in S_{d}$ tal que $\alpha_{1} \ldots \alpha_{k} \widehat{\beta}_{1}^{2}=1$.

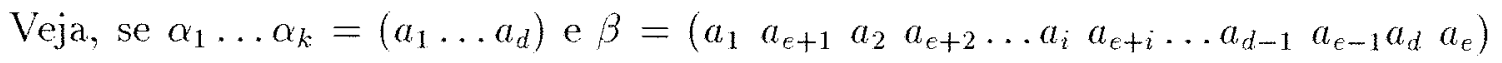
para $e=\frac{d+1}{2}$ é só fazer $\widehat{\beta}_{1}=\beta^{-1}$

2. Se $d$ é par. $\sum_{i=1}^{k} \nu\left(A_{i}\right)=(d-1)+r, \operatorname{com} d-1$ e $r$ impares. Fazendo a analise acima para este caso vamos ter que existem $\alpha_{i} \in A_{i}, i=1, \ldots, k$, tais que $\left\langle\alpha_{1} \ldots \alpha_{k-1}, \alpha_{k}\right\rangle$ age transitivamente sobre $\{1, \ldots, d\}$ e já que $\sum_{i=1}^{k} \nu\left(A_{1}\right)=d+(r-1) \operatorname{com} r-1$ par, pela proposição 3.2 .8 pode acontecer:

(a) $\alpha_{1} \ldots \alpha_{k} \in[d / 2, d / 2]$ se $A_{12 \ldots(k-1)}=A_{k}=[2, \ldots, 2]$. Neste caso existe um $d-$ ciclo $\widehat{\beta}_{1} \in S_{d}$ tal que $\alpha_{1} \ldots \alpha_{k} \widehat{\beta}_{1}^{2}=1$ : se $\alpha_{1} \ldots \alpha_{k}=\left(a_{1_{1}} \ldots a_{1_{d / 2}}\right)\left(a_{2_{2}} \ldots a_{2_{d / 2}}\right)$ e $\beta=\left(\begin{array}{llllll}a_{1_{1}} & a_{2_{1}} \ldots a_{1_{i}} & a_{2_{i}} \ldots a_{1_{d / 2}} & a_{2_{d / 2}}\end{array}\right)$ é só fazer $\widehat{\beta}_{1}=\beta^{-1}$

(b) Ou $\alpha_{1} \ldots \alpha_{k} \in[d-1,1], \log \circ \alpha_{1} \ldots \alpha_{k}$ é um $(d-1)$-ciclo par, portanto existe um $(d-1)$-ciclo $\widehat{\beta}_{1} \in S_{d}$, tal que $\alpha_{1} \ldots \alpha_{k} \widehat{\beta}_{1}^{2}=1$. Veja, se $\alpha_{1} \ldots \alpha_{k}=\left(a_{1} \ldots a_{d-1}\right)$

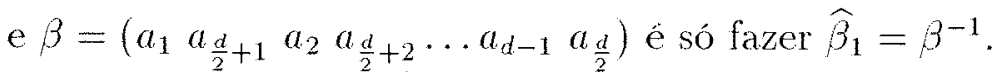

Assim, pelo corolário 2.4, sempre é possível garantir a existência de um revestimento ramificado $\phi: M \longrightarrow \mathbb{R} \mathrm{P}^{2}$ que realize $\mathscr{D}$ como dado de ramificação.

Para ver que $M$ pode ser não orientável, já que $\left\langle\alpha_{1}, \ldots, \alpha_{k}\right\rangle$ age transitivamente sobre $\{1, \ldots, d\}$, existe $\gamma \in\left\langle\alpha_{1}, \ldots, \alpha_{k}\right\rangle$ tal que $\gamma(\beta(1))=1, \log 0 \gamma \beta \in S_{d-1}$ e pelo lema 2.5 , $M$ é não orientável.

Vamos dizer que $\left(M, \phi, N, B_{\phi}\right)$ é um revestimento ramificado orientável (não orientável), se $M$ é orientável (não orientável). 
Dos resultados anteriores notamos que se $N$ é não orientável e $\mathscr{D}$ é um dado de ramificação virtual satisfazendo as condições de Hurwitz $\nu(\mathscr{D}) \equiv 0(\bmod 2)$ e $\nu(\mathscr{D}) \geq d-1$ podemos realizar $\mathscr{O}$. Mais que isso, se $d$ é impar, $M$ é não orientável, e se $d$ é par, sempre é possível achar um revestimento ramificado tal que $M$ seja năo orientável, mas nem sempre é possível realizar $\mathscr{D}$ com um revestimento ramificado orientável.

Exemplo $4.3 O$ dado de ramificação virtual $\mathscr{D}=\{[3,1]\}$ não é realizável mediante um. revestimento ramificado orientável da garrafa de Klein:

Seja $\mathbb{K}$ a garrafa de Klein e $y \in \mathbb{K}$. Para que exista um revestimento ramificado de grau 4 com ramificação localizada sobre $y$, é preciso definir $\Psi:\left\langle a, b, c \mid a b a b^{-1} c^{-1}=1\right\rangle \longrightarrow S_{4}$ tal que $[\widehat{a}, \widehat{b}]_{-} \in[3,1]$. Mas notemos que para garantir a orientabilidade da superficie de revestimento, nenhum ciclo de $\Psi(a)=\widehat{a}$ nem de $\Psi(b)=\widehat{b}$ pode ter comprimento impar. Isto é, precisamos que $\widehat{a}, \widehat{b} \in[2,2]$ o que implica, pela proposiçăo 3.8 .8 , que $[\widehat{a}, \widehat{b}]-\in[2,2]$, portanto é impossivel definir $\Psi$.

Proposição 4.4 Seja $d \in \mathbb{Z}^{+}$par, $N$ uma superfície fechada não orientável $e \mathscr{D}=$ $\left\{A_{1}, \ldots, A_{k}\right\}$ um dado de ramificação virtual. $\mathscr{D}$ é realizável por um revestimento ramificado orientável se e somente se para $1 \leq i \leq k$ é $A_{i}=\left[B_{i}, C_{i}\right]$ onde $B_{i}$ e $C_{i}$ são partiçôes de $d / 2$ tais que $\left\{B_{1}, \ldots, B_{k}, C_{1}, \ldots, C_{k}\right\}$ é dado de ramificação de um revestimento ramificado de grau d/2 do revestimento orientável duplo de $N$.

Demonstração: Seja $\left(M, \phi, N, B_{\phi}\right)$ um revestimento ramificado orientável de grau $d$. Consideremos os homomorfismos

$$
\begin{aligned}
\omega: \pi_{1}\left(N, z^{*}\right) & \longrightarrow\{+1,-1\} \\
\beta_{j} & \longmapsto-1 \\
\widehat{\omega}: \pi_{1}\left(\widehat{N}, z^{*}\right) & \longrightarrow\{+1,-1\} \\
\left\{v_{i}^{*}\right\} & \longmapsto+1 \\
\beta_{j} & \longmapsto-1
\end{aligned}
$$

$i=1, \ldots, k, j=1, \ldots, n$. 
$\widehat{M}=M-\phi^{-1}\left(B_{\phi}\right)$ é também orientável e pelo lema 2.5 ,

$$
\phi_{*}\left(\pi_{1}(\widehat{M})=\Psi^{-1}\left(S_{d-1}\right) \subset \text { Ker } \omega\right.
$$

onde $\Psi$ é a representação de Hurwitz associada a $\phi$.

Seja $(\widetilde{N}, \pi, N)$ o revestimento orientável duplo de $N$ determinado pelo Ker $\omega$. Tanto $(\widehat{M}, \phi, \widehat{N})$ quanto $\left(\pi^{-1}(\widehat{N}), \pi, \widehat{N}\right)$ são revestimentos não ramificados de $\widehat{N}$. Seja $\bar{N}=$ $\pi^{-1}(\widehat{N})$. Pela teoria dos revestimentos, sabemos que pode-se levantar $\phi$ para um revestimento de $\bar{N}$ se e somente se $\phi_{*}\left(\pi_{1}(\widehat{M})\right) \subset \pi_{*}\left(\pi_{1}(\bar{N})\right)$. Temos que Ker $\omega \subset$ Ker $\widehat{\omega}=$ $\pi_{*}\left(\pi_{1}(\bar{N})\right)$, logo por (4.2) podemos levantar $\phi$ para um revestimento não ramificado $\widetilde{\phi}: \widehat{M} \longrightarrow \bar{N}$ de grau $d / 2$ que faz o seguinte diagrama comutar

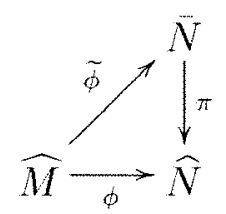

Seja $B_{\tilde{\phi}}=\pi^{-1}\left(B_{\phi}\right)$. Já que $B_{\widetilde{\phi}}$ é um conjunto discreto, podem-se preencher cada um destes "buraquinhos" em $\bar{N}$ do jeito usual. Com a comutatividade do diagrama (4.3) temos que cada $A_{i} \in \mathscr{D}$ pode escrever-se como $A_{i}=\left[B_{i}, C_{i}\right]$ onde $B_{i}$ e $C_{i}$ são partições de $d / 2$ e $\operatorname{assim}\left\{B_{1}, \ldots, B_{k}, C_{1}, \ldots, C_{k}\right\}$ é dado de ramificação de $\left(M, \widetilde{\phi}, \bar{N}, B_{\tilde{\phi}}\right)$.

Para a condição suficiente, suponha que cada $A_{i} \in \mathscr{D}$ é da forma $A_{i}=\left[B_{i}, C_{i}\right]$ onde $\left\{B_{1}, \ldots, B_{k}, C_{1}, \ldots, C_{k}\right\}$ é dado de ramificação de um revestimento ramificado $\left(M, \rho, \widetilde{N}, B_{\rho}\right)$ de grau $d / 2$. É claro que $M$ orientável, pois $\widetilde{N}$ é orientável, portanto $\left(M, \rho \circ \pi, N, \pi\left(B_{\rho}\right)\right)$ é um revestimento ramificado orientável de grau $d$.

Pela demonstração da proposição anterior, se $d$ é par os revestimentos ramificados orientáveis de grau $d$ do plano projetivo, dependem dos revestimentos ramificados de grau $d / 2$ do revestimento orientável duplo de $\mathbb{R}^{2}$. Isto é, estudar a realização de dados de ramificação virtuais sobre $\mathbb{R} P^{2}$ mediante revestimentos ramificados orientáveis, equivale a estudar a realização de dados de ramificação "especiais" da esfera que como vamos ver não é um problema trivial.

As condições de Hurwitz para o caso da esfera são $\nu(\mathscr{D}) \equiv 0(\bmod 2)$ e $\nu(\mathscr{D}) \geq 2 d-2$, em geral elas não são suficientes para realizar $\mathscr{D}$. 
Exemplo 4.5 O conjunto $\mathscr{D}=\{[2,2],[2,2],[3,1]\}$ de partições do 4 satisfaz as condiçóes de Hurwitz mas não é realizável, pois é impossivel definir uma representação de Hurwitz $\Psi: \pi_{1}\left(\mathbb{S}^{2}-\left\{x_{1}, x_{2}, x_{3}\right\}\right) \longrightarrow S_{4}$, jâ que, pela proposição 3.2 .8 , se $\alpha, \beta \in[2,2]$, então $\alpha \beta \in[2,2]$, ou se $\beta \in[3,1]$ então $\alpha \beta \in[3,1]$.

Lembremos que para garantir a realização de um dado de ramificação virtual $\mathscr{D}=$ $\left\{A_{1}, \ldots, A_{k}\right\}$ mediante um revestimento ramificado conexo da esfera precisamos da existência de representantes $\alpha_{i} \in A_{i}$ tais que $\alpha_{1} \ldots \alpha_{k}=1$ e que a ação de $\left\langle\alpha_{1}, \ldots, \alpha_{k}\right\rangle$ sobre $\{1, \ldots, d\}$ seja transitiva.

Proposição 4.6 conjunto $\mathscr{D}=\left\{A_{1}, \ldots, A_{k}\right\}$ de partições de $d$, com $A_{i}=[d]$ para algum $i \in\{1, \ldots, k\}$, é realizável por um revestimento ramificado de grau d da esfera se $e$ somente se satisfaz as condiçoes de Hurwitz.

Demonstração: A condição necessária está feita. Vamos supor então que $\mathscr{D}$ satisfaz as condições de Hurwitz isto é, $\nu(\mathscr{D}) \geq 2 d-2$ e $\nu(\mathscr{D}) \equiv 0(\bmod 2)$.

Se $k=2, \mathscr{D}=\left\{A_{1}, A_{2}\right\} \operatorname{com} \nu\left(A_{i}\right)=d-t_{i}, i=1,2$. Mas pela hipótese $\nu\left(A_{1}\right)+$ $\nu\left(A_{2}\right) \geq 2 d-2$ donde $2 d-\left(t_{1}+t_{2}\right) \geq 2 d-2$, portanto $t_{1}+t_{2} \leq 2$ e assim $t_{1}=t_{2}=1$, isto é, $A_{1}=A_{2}=[d]$. Peguemos $\alpha_{1}=(1 \ldots d)$ e $\alpha_{2}=\alpha_{1}^{-1}$, é claro que $\alpha_{1} \alpha_{2}=1$.

Se $k=3, \mathscr{D}=\left\{A_{1}, A_{2}, A_{3}\right\}$ é tal que $\nu\left(A_{1}\right)+\nu\left(A_{2}\right)+\nu\left(A_{3}\right) \geq 2 d-2>d-1$, e já que pela hipótese existe $i \in\{1,2,3\}$ tal que $A_{i}=[d]$, então existe $i \in\{1,2,3\}$ tal que $\nu\left(A_{i}\right)=d-1$. Sem perda de generalidade vamos supor $i=3$, donde

$$
\nu\left(A_{1}\right)+\nu\left(A_{2}\right) \equiv-d+1 \quad(\bmod 2)
$$

e já que $d+1 \equiv-d+1(\bmod 2)$ pelo corolário 3.2 .7 , existem $\alpha_{1} \in A_{1}, \alpha_{2} \in A_{2}$ tais que $\nu\left(\alpha_{1} \alpha_{2}\right)=d-1=\nu\left(A_{3}\right)$. Assim, é só pegar $\alpha_{3}=\left(\alpha_{1} \alpha_{2}\right)^{-1}$.

Para $k>3$, por indução. Vamos assumir $A_{k}=[d]$. Pode acontecer:

1. Que existam $i, j$ tais que $\nu\left(A_{i}\right)+\nu\left(A_{j}\right) \leq d-1$, para $i \neq j$. Sem perda de generalidade podemos supor $\nu\left(A_{1}\right)+\nu\left(A_{2}\right) \leq d-1$, pela proposição 3.2 .5 existem $\alpha_{1} \in A_{1}, \alpha_{2} \in A_{2}$ tais que $\nu\left(\alpha_{1} \alpha_{2}\right)=\nu\left(\alpha_{1}\right)+\nu\left(\alpha_{2}\right)$. Seja $A=\left\|\alpha_{1} \alpha_{2}\right\|$. Então 
$\mathscr{D}^{\prime}=\left\{A, A_{3}, \ldots, A_{k}\right\}$ satisfaz a hipóteses da proposição e pela indução, existe $\alpha \in A$ e $\alpha_{i} \in A_{i}, 3 \leq i \leq k$ que realizam $\mathscr{D}^{\prime}$. Isto é, existem $\alpha \in A, \alpha_{i} \in A_{i}, 3 \leq i \leq k$, tais que $\alpha \alpha_{3} \ldots \alpha_{k}=1$. E claro que $\alpha$ e $\alpha_{1} \alpha_{2}$ são conjugados, portanto existe $\gamma \in S_{d}$ tal que $\alpha=\gamma \alpha_{1} \alpha_{2} \gamma^{-1} \operatorname{logo} \gamma \alpha_{1} \gamma^{-1}, \gamma \alpha_{2} \gamma^{-1}, \alpha_{3}, \ldots, \alpha_{k}$ realiza $\mathscr{D}$.

2. Que $\nu\left(A_{i}\right)+\nu\left(A_{j}\right) \geq d$, para todo $i \neq j$. Seja $\nu\left(A_{1}\right)+\nu\left(A_{2}\right)=d+R, R \geq 0$.

(a) Se $R$ for ímpar, $\nu\left(A_{1}\right)+\nu\left(A_{2}\right)=(d-1)+(R+1)$, e já que $R+1$ é par, pela proposição 3.2 .6 podemos escolher $\alpha_{1} \in A_{1}, \alpha_{2} \in A_{2}$ tais que $\left\langle\alpha_{1}, \alpha_{2}\right\rangle$ age transitivamente sobre $\{1, \ldots, d\}$ e $\nu\left(\alpha_{1} \alpha_{2}\right)=d-1$.

(b) Se $R$ for par, pela proposição $3.2 .8, \nu\left(\alpha_{1} \alpha_{2}\right)=d-2$.

Logo sempre vamos ter $\nu\left(\alpha_{1} \alpha_{2}\right) \geq d-2$ e $\nu\left(\alpha_{1} \alpha_{2}\right) \equiv \nu\left(\alpha_{1}\right)+\nu\left(\alpha_{2}\right)(\bmod 2)$. Seja $A=\left\|\alpha_{1} \alpha_{2}\right\|$ e $\mathscr{D}^{\prime}=\left\{A, A_{3}, \ldots, A_{k}\right\}$. Então $\nu\left(\mathscr{D}^{\prime}\right) \equiv 0(\bmod 2) \mathrm{e}$

$$
\begin{aligned}
\nu\left(\mathscr{D}^{\prime}\right) & =\nu\left(\alpha_{1} \alpha_{2}\right)+2 d-2-\left(\nu\left(A_{1}\right)+\nu\left(A_{2}\right)\right) \\
& \geq 2 d-2+2 q \\
& \geq 2 d-2
\end{aligned}
$$

onde $\nu\left(\alpha_{1} \alpha_{2}\right)-\left(\nu\left(A_{1}\right)+\nu\left(A_{2}\right)\right)=2 q$. Assim $\mathscr{D}^{\prime}$ é realizável por indução e analogamente ao caso anterior, é possível realizar $\mathscr{D}$.

Proposição 4.7 O conjunto $\mathscr{D}=\left\{A_{1}, \ldots, A_{k}\right\}$ de partiçöes de d, com $A_{i}=[d-1,1]$ para algum $i \in\{1, \ldots, k\}$, é realizável por um revestimento ramificado da esfera se e somente se satisfaz as condiçoes de Hurwitz e é diferente de:

(1) $\{[2,2], \ldots,[2,2],[3,1]\}$ para $d=4$ ek $\geq 3$.

(2) $\{[2, \ldots, 2],[2, \ldots, 2],[d-1,1]\}$ para $d=2 r \in k=3$.

Demonstração: Os dados de ramificação virtuais dados em (1) e (2) não são realizáveis, pois no primeiro caso tem-se que as permutações com estrutura cíclica $[2,2]$ geram o 2 Sylow de $A_{4}$ e este subgrupo de $S_{4}$ não contém 3-ciclos. E o segundo caso não é realizável 
pela proposição 3.2 .8 , pois para todo $\alpha, \beta \in[2, \ldots, 2]$ temos que $\alpha \beta \in[d / 2, d / 2]$. Assim as condições necessárias foram demonstradas.

Resta provar que qualquer outro conjunto de partições satisfazendo as condições de Hurwitz é realizável. Para começar, da hipótese temos que $k \geq 3$, pois se $k=1, \mathscr{D}=$ $\{[d-1,1]\}$ e $\nu(\mathscr{D})=d-2<2 d-2$ o que contradiz uma das condições de Hurwitz. Se $k=2, \mathscr{D}=\left\{A_{1}, A_{2}\right\}$. Vamos supor $A_{1}=[d-1,1]$ e $\nu\left(A_{2}\right)=d-t, t>0$. Logo $\nu(\mathscr{D})=2 d-(t+2) \geq 2 d-2$, donde $t \leq 0$, absurdo.

Seja então $k=3$. Temos $\mathscr{D}=\left\{A_{1}, A_{2}, A_{3}\right\}$ satisfazendo as condições de Hurwitz e, sem perda de generalidade, $A_{3}=[d-1,1]$. Assim, $\nu\left(A_{1}\right)+\nu\left(A_{2}\right) \geq 2 d-2-\nu\left(A_{3}\right)$, isto é, $\nu\left(A_{1}\right)+\nu\left(A_{2}\right)=(2 d-2+2 q)-(d-2)$ para $q \geq 0$ pois $\nu(\mathscr{D})$ é par. Portanto $\nu\left(A_{1}\right)+\nu\left(A_{2}\right)=d+2 q$. Pela proposição 3.2.8, existem $\alpha_{1} \in A_{1}, \alpha_{2} \in A_{2}$ tais que $\left\langle\alpha_{1}, \alpha_{2}\right\rangle$ age transitivamente sobre $\{1, \ldots, d\}$ e $\alpha_{1} \alpha_{2} \in[d-1,1]$, pois $\mathscr{D}$ é diferente do conjunto em (2). Fazendo $\alpha_{3}=\left(\alpha_{1} \alpha_{2}\right)^{-1}$ é claro que $\alpha_{1} \alpha_{2} \alpha_{3}=1$, com $\alpha_{3} \in A_{3}$, e este produto realiza $\mathscr{D}$.

Para $k \geq 4$ vamos provar por indução e usaremos o método de redução da demonstração da proposição anterior. Assumamos que $A_{k}=\{[d-1,1]\}$. Pelas proposições 3.2.5, 3.2.6 e 3.2.8, existem $\alpha_{1} \in A_{1}, \alpha_{2} \in A_{2}$ tais que $\nu\left(\alpha_{1} \alpha_{2}\right)=\nu\left(\alpha_{1}\right)+\nu\left(\alpha_{2}\right)$ ou $\nu\left(\alpha_{1} \alpha_{2}\right) \geq d-2 \mathrm{e}$ além disso temos que $\nu\left(\alpha_{1} \alpha_{2}\right) \equiv \nu\left(\alpha_{1}\right)+\nu\left(\alpha_{2}\right)(\bmod 2)$.

Se $d=4$, podemos pegar $\alpha_{1} \alpha_{2} \notin[2,2]$ pois $\mathscr{D}$ é diferente do conjunto em (1). Seja $\mathscr{D}^{\prime}=\left\{\left\|\alpha_{1} \alpha_{2}\right\|, A_{3}, \ldots, A_{k}\right\}$, então $\nu\left(\mathscr{D}^{\prime}\right)=\nu(\mathscr{D})$ ou $\nu\left(\mathscr{D}^{\prime}\right) \geq 2 d-2$, pela hipótese de indução $\mathscr{D}^{\prime}$ é realizável e da mesma forma que na demonstração da proposição anterior podemos concluir que $\mathscr{D}$ é realizável.

Teorema 4.8 Seja $d \neq 4$. Um dado de ramificação virtual $\mathscr{D}=\left\{A_{1}, \ldots, A_{k}\right\}$ é realizável por um revestimento ramificado de grau d da esfera se $\nu(\mathscr{D}) \equiv 0(\bmod 2)$ e $\nu(\mathscr{D}) \geq$ $3(d-1)$.

Demonstração: $\mathrm{O}$ caso $d=2$ é trivial, pois neste caso para todo $A_{i} \in \mathscr{D}, A_{i}=[2]$ e as condições do teorema implicam que $k$ é par. Assim podemos definir a seguinte 
representação

$$
\begin{aligned}
\left.\left.\Psi:\left\langle\left\{v_{1}^{*}\right\}, \ldots,\left\{v_{k}^{*}\right\}\right|\left\{v_{1}\right\}^{*} \ldots\left\{v_{k}\right\}^{*}=1\right\}\right\rangle & \longrightarrow S_{2} \\
\left\{v_{i}^{*}\right\} & \longmapsto \sigma_{i}=(12)
\end{aligned}
$$

para $i=\{1, \ldots, k\}, \operatorname{logo} \sigma_{1} \ldots \sigma_{k}=1$.

Seja $d \geq 3$. O caso $k \leq 2$ é vazio. Veja, se $k=2, \mathscr{D}=\left\{A_{1}, A_{2}\right\}$ e $\nu(\mathscr{D})=2 d-\left(t_{1}+t_{2}\right)$. Já que $\nu(\mathscr{D}) \equiv 0(\bmod 2)$ então $t_{1}+t_{2} \equiv 0(\bmod 2)$, além disso, $2 d-\left(t_{1}+t_{2}\right) \geq 2 d-(3-d)$ logo $t_{1}+t_{2} \leq 3-d$, isto é $t_{1}+t_{2} \leq 0$, absurdo. Se $k=1, d-t \equiv 0(\bmod 2)$, isto quer dizer que $2 \mid(d-t)$, mas $d-t \geq 3 d-3$ portanto $0<t \leq 3-2 d$, impossível porque no mínimo $d=3$.

Seja $k=3, \mathscr{D}=\left\{A_{1}, A_{2}, A_{3}\right\}$ e $\nu(\mathscr{D})=3 d-\left(t_{1}+t_{2}+t_{3}\right) \geq 3 d-3 \operatorname{logo} t_{1}+t_{2}+t_{3} \leq 3$ e já que $t_{i}>0, i=1,2,3$, temos que $t_{i}=1$ donde $A_{i}=[d]$. Agora, como $t_{1}+t_{2}+t_{3} \equiv d$ $(\bmod 2)$ então $d$ é ímpar. Neste caso definimos $\alpha_{1}=\alpha_{2}=(12 \ldots d)$ e $\alpha_{3}=\left(\alpha_{1} \alpha_{2}\right)^{-1}$.

Para $k \geq 4$, demonstraremos por indução. Pode acontecer:

1. Que existam $i, j$ tais que $\nu\left(A_{i}\right)+\nu\left(A_{j}\right) \leq d-1, i \neq j$. Sem perda de generalidade podemos supor $\nu\left(A_{1}\right)+\nu\left(A_{2}\right) \leq d-1$ isto é $\nu\left(A_{1}\right)+\nu\left(A_{2}\right)=d-t, t \geq 1 \mathrm{e}$ pela proposição 3.2 .5 existem $\alpha_{1} \in A_{1}, \alpha_{2} \in A_{2}$ tais que $\nu\left(\alpha_{1} \alpha_{2}\right)=\nu\left(\alpha_{1}\right)+\nu\left(\alpha_{2}\right)$. Seja $A=\left\|\alpha_{1} \alpha_{2}\right\|$ e $\mathscr{D}^{\prime}=\left\{A, A_{3} \ldots, A_{k}\right\}$. Por indução $\mathscr{D}^{\prime}$ é realizável e portanto $\gamma \alpha_{1} \gamma^{-1}, \gamma \alpha_{2} \gamma^{-1}, \alpha_{3}, \ldots, \alpha_{k}$ realiza $\mathscr{D}$, para $\gamma \in S_{d}$, pois $\alpha \in A$ e $\alpha_{1} \alpha_{2}$ são conjugados.

2. Que $\nu\left(A_{i}\right)+\nu\left(A_{j}\right) \geq d$ para todo $i \neq j$. Então $2 d-\left(t_{i}+t_{j}\right) \geq d \operatorname{logo} d \geq t_{i}+t_{j}$, portanto $t_{1}+t_{2} \in\{2,3\}$ donde $t_{1}=1$ ou $t_{2}=1$ o que implica $A_{1}=[d]$ ou $A_{2}=[d]$, logo $\mathscr{D}$ é realizável pela proposição 4.6 .

Podemos supor então que $\nu\left(A_{i}\right) \leq d-2$. Temos dois sub-casos:

(a) Algum $A_{i} \neq[2, \ldots, 2]$. Suponhamos $A_{1} \neq[2, \ldots, 2]$ e $\nu\left(A_{1}\right)$ minimal entre os 
$\nu\left(A_{j}\right) \operatorname{com} A_{j} \neq[2, \ldots, 2]$. Agora

$$
\begin{aligned}
\nu\left(A_{1}\right)+\nu\left(A_{2}\right) & =2 d-4-q, q \geq 0 \\
& =(d-1)+(1+d-4-q) \\
& =d+(d-4-q) .
\end{aligned}
$$

Se $1+d-4+q$ é impar, $d-4+q$ é par e pela proposição 3.2 .8 , existem $\alpha_{1} \in A_{1}, \alpha_{2} \in A_{2}$ tais que $\left\langle\alpha_{1}, \alpha_{2}\right\rangle$ age transitivamente sobre $\{1, \ldots, d\}$ e $\alpha_{1} \alpha_{2} \in[d-1,1]$.

Se $1+d-4+q$ é par, pela proposição 3.2 .6 existem $\alpha_{1} \in A_{1}, \alpha_{2} \in A_{2}$ tais que $\left\langle\alpha_{1}, \alpha_{2}\right\rangle$ age transitivamente sobre $\{1, \ldots d\}$ e $\nu\left(\alpha_{1} \alpha_{2}\right)=d-1$ portanto $\alpha_{1} \alpha_{2} \in[d]$.

Seja $A=\left\|\alpha_{1} \alpha_{2}\right\|$ e $\mathscr{D}^{\prime}=\left\{A, A_{3}, \ldots, A_{k}\right\}$. De outro lado

$$
\nu\left(\mathscr{D}^{\prime}\right) \geq(d-2)+\frac{(k-2) d}{2} \geq 2 d-2
$$

já que no máximo existe um $i$ tal que $\nu\left(A_{i}\right)<d / 2$ e $k \geq 4$. Agora $\mathscr{D}^{i}$ é realizável exceto se $k=4$ e $A_{3}=A_{4}=[2, \ldots, 2]$, neste caso $\nu(\mathscr{D}) \leq 2(d-2)+(2 d) / 2=$ $3 d-4<3(d-1)$ pois $\nu\left(A_{i}\right) \leq d-2, i \in\{1,2\}$ e $\nu\left(A_{j}\right)=d / 2, j \in\{3,4\}$, contradizendo o que assumimos.

Alterando a escolha de $\alpha \in A$ por o produto de $\gamma^{-1} \alpha_{1} \gamma$ e $\gamma^{-1} \alpha_{2} \gamma$ para um $\gamma \in S_{d}$, se realiza $\mathscr{D}$.

(b) Que $A_{i}=[2, \ldots, 2]$ para $i=1, \ldots, k$. Já que $\nu(\mathscr{D})$ é par e $3(d-1)$ é ímpar, então $\nu(\mathscr{D})=k d / 2>3(d-1)$, donde $k>\frac{6(d-1)}{d} \geq 5$ pois $d$ é par e $d \geq 6$. Temos então que $\nu\left(A_{1}\right)+\nu\left(A_{2}\right)=d$, pela proposição 3.2 .8 existem $\alpha_{1} \in A_{1}, \alpha_{2} \in A_{2}$ tais que $\left\langle\alpha_{1}, \alpha_{2}\right\rangle$ age transitivamente sobre $\{1, \ldots, d\}$ e $\alpha_{1} \alpha_{2} \in[d / 2, d / 2]$.

Seja $A=\left\|\alpha_{1} \alpha_{2}\right\|$ e $\mathscr{D}^{\prime}=\left\{A, A_{3}, \ldots, A_{k}\right\}$.

$$
\begin{aligned}
\nu(A)+\nu\left(A_{3}\right) & =d-2+d / 2 \\
& =d+(d / 2-2) \\
& =(d-1)+(d / 2-1) .
\end{aligned}
$$

Se $d / 2-2$ é par, pela proposição 3.2 .8 existe $\alpha_{3} \in A_{3}$ tal que $\alpha_{1} \alpha_{2} \alpha_{3} \in[d-1,1]$. 
Se $d / 2-2$ é ímpar, pela proposição 3.2 .6 existe $\alpha_{3} \in A_{3}$ tal que $\alpha_{1} \alpha_{2} \alpha_{3}=[d]$. Já que $k \geq 6$ as duas proposições anteriores garantem que o dado de ramificação virtual $\left\{\left\|\alpha_{1} \alpha_{2} \alpha_{3}\right\|, A_{4}, \ldots, A_{k}\right\}$ é realizável e daqui $\mathscr{D}$ é realizável.

Corolario 4.9 Se $d \neq 4$ existe só um número finito de dados de ramificação virtuais năo realizáveis mediante revestimentos ramificados de grau d da esfera.

Demonstração: Só existe um número finito de dados de ramificação virtuais $\mathscr{D}$ tais que $\nu(\mathscr{D})<3(d-1)$

Corolario 4.10 Para $d=4$, um dado de ramificaçăo virtual $\mathscr{D}=\left\{A_{1}, \ldots, A_{k}\right\}$ é realizável mediante um revestimento ramificado de grau d da esfera se e somente se $\nu(\mathscr{D}) \geq$ $2 d-2=6, \nu(\mathscr{D}) \equiv 0(\bmod 2)$ e $\mathscr{D} \neq\{[2,2],[2,2], \ldots,[2,2],[3,1]\}$.

Demonstração: As condições necessárias já foram provadas. Para as condições suficientes vimos que não é possível realizar $\{[2,2], \ldots,[2,2],[3,1]\}$. Se $\mathscr{D}$ contém um $[4]$ ou um $[3,1]$ pelas proposições 4.6 e $4.7 \mathscr{D}$ é realizável. Senão, então $\mathscr{D}$ só contém $[2,2]$ e/ou $[2,1,1]$. Se ambos aparecem, vamos supor que $A_{1}=[2,2]$ e $A_{2}=[2,1,1]$, logo $\nu\left(A_{1}\right)+\nu\left(A_{2}\right)=3=4-1$. Pelo corolário 3.2.7 existem $\alpha_{1} \in A_{1}, \alpha_{2} \in A_{2}$ tais que $A=\left\|\alpha_{1} \alpha_{2}\right\|=[4]$. assim, pela proposição $4.6, \mathscr{D}^{\prime}=\left\{A, A_{3}, \ldots, A_{k}\right\}$ é realizável e portanto $\mathscr{D}$ também. Se só aparecem $[2,1,1]$, já que $\nu(\mathscr{D}) \geq 6$, $\mathscr{D}$ tem no mínimo 6 partições. Temos então $\nu\left(A_{1}\right)+\nu\left(A_{2}\right)=4-2$ e pela proposição 3.2 .5 existem $\alpha_{1} \in A_{1}, \alpha_{2} \in A_{2}$ tais que $\nu\left(\alpha_{1} \alpha_{2}\right)=4-2$. Seja $A=\left\|\alpha_{1} \alpha_{2}\right\|$, donde $\nu(A)+\nu\left(A_{3}\right)=2+1=3=4-1$ e pelo corolário 3.2.7 existe $\alpha_{3} \in A_{3}$ tal que $\alpha_{1} \alpha_{2} \alpha_{3} \in[d]=[4]$, portanto $\mathscr{D}$ é realizável. Se só aparecem $[2,2], \nu\left(A_{1}\right)+\nu\left(A_{2}\right)=4$ e pela proposição 3.2 .8 existem $\alpha_{1} \in A_{1}, \alpha_{2} \in A_{2}$ tais que $\alpha_{1} \alpha_{2} \in[2,2]$. Seja $A=\left\|\alpha_{1} \alpha_{2}\right\|$. Se $k=3, \alpha_{3}=\left(\alpha_{1} \alpha_{2}\right)^{-1}$ implica que $\mathscr{D}$ é realizável. Se $k>3$, por indução $\mathscr{D}^{\prime}$ é realizável e portanto $\mathscr{D}$ é realizável.

Proposição 4.11 Se $d=a b$ com $a, b>1$, entäo embora $\mathscr{D}=\{[a, \ldots, a],[b+1,1, \ldots, 1]$, $[a, a(b-1)]\}$ satisfaça as condiçöes de Hurwitz, $\mathscr{D}$ nä̀ é realizável por nenhum revestimento ramificado da esfera. 
Demonstração: O plano para fazer a prova é o seguinte, fixar $\beta=(12 \ldots b+1) \epsilon$ $[b+1,1, \ldots, 1]$, considerar todos os possíveis $\alpha \in[a, \ldots, a]$ com $\langle\alpha, \beta\rangle$ transitivo e mostrar que $\alpha \beta$ não pode cair em $[a, a(b-1)]$.

A transitividade requer que nenhum dos $b a$-ciclos de $\alpha$ envolva só dados majores que $b+1$, pois não teríamos como conectar $b+1 \operatorname{com} b+i$ por exemplo. Segue então que $(b-1) a$-ciclos de $\alpha$ contêm exatamente um dado menor ou igual a $b+1$ e um deles contém dois. Sem perda de generalidade podemos assumir que

$$
\alpha=(1 \ldots j \ldots)(2 \ldots) \ldots(j-1 \ldots)(j+1 \ldots) \ldots(b+1 \ldots)
$$

para algum $j \leq b+1$. Assim

$$
\alpha \beta=(\underbrace{1 \ldots}_{u} \underbrace{j+1 \ldots}_{a} \underbrace{j+2 \ldots}_{a} \quad \cdots \underbrace{b+1 \ldots}_{a})(\underbrace{j \cdots}_{v} \underbrace{2 \ldots}_{a} \quad \cdots \underbrace{j-1 \ldots}_{a})
$$

onde $u+v=a$ e $u, v \geq 1 . A \operatorname{ssim}, \alpha \beta \in[x, d-x]$ onde $x \equiv u(\bmod a), d-x \equiv v$ $(\bmod a)$. Em particular nem $x$ nem $d-x$ são divisíveis por $a$, e por isto $\alpha \beta \notin[a, a(b-1)]$.

Teorema 4.12 Se $d \neq 8$ é par, então um dado de ramificação virtual $\mathscr{D}=\left\{A_{1}, \ldots, A_{k}\right\}$ com $A_{i}=\left\{B_{i}, C_{i}\right\}, \in B_{i}, C_{i} \in \pi(d / 2)$, é realizável por um revestimento ramificado conexo orientável do $\mathbb{R P}^{2}$ se $\nu(\mathscr{D}) \geq 3((d / 2)-1) \in \nu(\mathscr{D}) \equiv 0(\bmod 2)$.

Demonstração: Seja $d=2 d^{\prime}$. Escolhamos um refinamento $B_{i} \cup C_{i}$ de $A_{i}, 1 \leq i \leq k$. Então para $\mathscr{D}^{\prime}=\left\{B_{1}, C_{1}, \ldots, B_{k}, C_{k}\right\}$ temos

$$
\nu\left(\mathscr{D}^{\prime}\right)=\sum_{i=1}^{k}\left(\nu\left(B_{i}\right)+\nu\left(C_{i}\right)\right)=\sum_{i=1}^{k} \nu\left(A_{i}\right) \geq 3\left(d^{\prime}-1\right)
$$

Pelo teorema $4.8 \mathscr{P}^{\prime}$ é realizável por um revestimento ramificado conexo de grau $d^{\prime}$ da esfera. Assim, fazendo a composição com o revestimento $\mathbb{S}^{2} \longrightarrow \mathbb{R} \mathrm{P}^{2}$ podemos completar a prova como na proposição 4.4 .

Temos como consequência que para $d \neq 8$ par, só um número finito de dados de ramificação virtuais, onde cada partição pode refinar-se e assim tornar-se um elemento de 
$\pi(d / 2)$, não são realizáveis por revestimentos ramificados conexos e orientáveis de grau $d$ do plano projetivo.

Corolario 4.13 Para $d=8$, as coleçoes $\mathscr{D}_{k}=\{[3,1,1,1,1,1],[2,2,2,2], \ldots,[2,2,2,2]\}$ e $\mathscr{E}_{k}=\{[3,1,2,2],[2,2,2,2], \ldots,[2,2,2,2]\}$ para $k \geq 2$ e satisfazendo $\nu\left(\mathscr{E}_{k}\right)>\nu\left(\mathscr{D}_{k}\right)=$ $4 k-2 \geq 6=d-2$ não são realizáveis por nenhum revestimento ramificado conexo $e$ orientável de $\mathbb{R}^{2}$.

Demonstração: O único refinamento do tipo [4,4] das partições acima é aquele que o corolário 4.10 proíbe. 


\section{Capítulo 5}

\section{Revestimentos Ramificados Primitivos}

Estudamos, neste capítulo, a realização de dados da ramificação virtuais mediante revestimentos ramificados primitivos, e uma generalização deste problema, quando $N$ é fechada e diferente da esfera. Ver [BGKZ2] e [EKS].

Todo revestimento ramificado $\left(M, \phi, N, B_{\phi}\right)$ de grau finito $d$ determina um subgrupo de $\pi_{1}(N)$ de índice finito, $\phi_{*}\left(\pi_{1}(M)\right)$.

Questão 2 Seja $H \subset \pi_{1}(N)$ um subgrupo de indice $l$ finito, $d \in \mathbb{Z}^{+}$finito, $\epsilon \mathscr{D}=$ $\left\{A_{1}, \ldots, A_{k}\right\}$ um dado de ramificação virtual. Será que existe um revestimento ramificado conero $\left(M, \phi, N, B_{\phi}\right)$ de grau d que realize $\mathscr{D}$ e tal que $\phi_{*}\left(\pi_{1}(M)\right)=H$ ?

Notemos que quando $N=\mathbb{R} \mathrm{P}^{2}$ e $H=\pi_{1}(N)$ a resposta à questão acima é afirmativa, pois um conjunto de partições de $d$ satisfazendo as condições de Hurwitz $\nu(\mathscr{D}) \equiv 0(\bmod 2)$ e $\nu(\mathscr{D}) \geq d-1$, sempre pode-se realizar com um revestimento ramificado não orientável $\left(M, \phi, N, B_{\phi}\right)$ e, neste caso, $\phi_{*}\left(\pi_{1}(M)\right)=\pi_{1}(N) \cong \mathbb{Z}_{2}$. Pois se for $\phi_{*}\left(\pi_{1}(M)\right)=\{0\}$, seria possível levantar $\phi$ a um revestimento ramificado de $\mathbb{S}^{2}$, o que é absurdo já que $M$ é não orientável.

Uma função contínua chama-se primitiva se o homomorfismo induzido entre os grupos fundamentais é sobrejetor. Assim, vamos dizer que $\left(M, \phi, N, B_{\phi}\right)$ é um revestimento ramificado primitivo se $\phi_{\times}\left(\pi_{1}(M)\right)=\pi_{1}(N)$. 
Lembremos que o subgrupo de isotropia de um elemento $z_{i} \in \phi^{-1}\left(z^{*}\right)=\left\{z_{1}, \ldots, z_{d}\right\}$ esta dado por

$$
I_{\pi_{1}\left(\hat{N}, z^{*}\right)}\left(z_{i}\right)=\left\{a \in \pi_{1}\left(\widehat{N}, z^{*}\right) \mid z_{i} \cdot a=z_{i}\right\} .
$$

Agora, se $\Psi$ é a representação de Hurwitz associada a $\phi,(5.1)$ pode-se escrever assim:

$$
I_{\Psi}(i)=\left\{a \in \pi_{1}\left(\widehat{N}, z^{*}\right) \mid i . \widehat{a}=i\right\} .
$$

onde $\widehat{a}=\Psi(a)$.

Teorema 5.1 Seja $N$ uma superficie compacta e $\left(M, \phi, N, B_{\phi}\right)$ um revestimento ramificado de grau d. Então $\phi_{*}\left(\pi_{1}\left(M, z_{1}\right)\right)=i_{*}\left(I_{\Psi}(1)\right)$, onde $i_{*}$ é o homomorfismo de grupos induzido pela inclusẫo $i: \widehat{N} \hookrightarrow N$.

Demonstração: Seja $\widehat{M}=M-\phi^{-1}\left(B_{\phi}\right)$ e $\widehat{\phi}=\left.\phi\right|_{\widehat{M}}$. Sabemos que $\widehat{\phi}: \widehat{M} \longrightarrow \widehat{N}$ é um revestimento não ramificado de grau $d$ e que $\widehat{\phi}_{*}\left(\pi_{1}\left(\widehat{M}, z_{1}\right)\right)=I_{\Psi}(1) \subset \pi_{1}\left(\widehat{N}, z^{*}\right) .{ }^{1}$ Por outro lado, os homomorfismos

$$
\begin{aligned}
& i_{*}: \pi_{1}\left(\widehat{N}, z^{*}\right) \longrightarrow \pi_{1}\left(N, z^{*}\right) \\
& j_{*}: \pi_{1}\left(\widehat{M}, z_{1}\right) \longrightarrow \pi_{1}\left(M, z_{1}\right)
\end{aligned}
$$

induzidos pelas respectivas inclusões, são sobrejetores. Portanto

$$
\begin{aligned}
\phi_{*}\left(\pi_{1}\left(M, z_{1}\right)\right) & =\phi_{*}\left(j_{*}\left(\pi_{1}\left(\widehat{M}, z_{1}\right)\right)\right) \\
& =i_{*}\left(\widehat{\phi}_{*}\left(\pi_{1}\left(\widehat{M}, z_{1}\right)\right)\right) \\
& =i_{*}\left(I_{\Psi}(1)\right) .
\end{aligned}
$$

Corolario 5.2 As seguintes afirmações são equivalentes:

1. $\phi$ é primitiva.

2. A composição $I_{\Psi}(k) \hookrightarrow \pi_{1}\left(\widehat{N}, z^{*}\right) \longrightarrow \pi_{1}\left(N, z^{*}\right)$ é sobrejetora para todo $k \in\{1, \ldots, d\}$.

3. A composição $I_{\Psi}(k) \hookrightarrow \pi_{1}\left(\widehat{N}, z^{*}\right) \longrightarrow \pi_{1}\left(N, z^{*}\right)$ é sobrejetora para algum símbolo $k \in\{1, \ldots, d\}$.

\footnotetext{
${ }^{1}$ Ver [Ma], capítulo $V$, seção 7 , página 162 .
} 
Corolario 5.3 Seja $H$ um subgrupo de $\pi_{1}\left(N, z^{*}\right)$. As seguintes afirmaçôes são equivalentes:

1. $\phi_{*}\left(\pi_{1}\left(M, z_{1}\right)\right)=H$.

2. A imagem da composiçẫo $I_{\Psi}(1) \hookrightarrow \pi_{1}\left(\widehat{N}, z^{*}\right) \longrightarrow \pi_{1}\left(N, z^{*}\right) \dot{\epsilon} H$.

Os teoremas $3.3 .3,3.3 .5$ junto com o corolário 5.2 garantem a realização de revestimentos ramificados primitivos sobre o toro ou a garrafa de Klein dado $\mathscr{D}=\{A\}$. Pois já que

$$
\pi_{1}\left(\widehat{N}, z^{*}\right)= \begin{cases}\left\langle a, b, c \mid[a, b] c^{-1}=1\right\rangle & \widehat{N}=\text { toro menos um ponto } \\ \left\langle a, b, c \mid[a, b]_{-} c^{-1}=1\right\rangle & \widehat{N}=\text { garrafa de Klein menos um ponto }\end{cases}
$$

basta definir

$$
\begin{aligned}
\Psi: \pi_{1}\left(\widehat{N}, z^{*}\right) & \longrightarrow S_{d} \\
a & \longmapsto \widehat{a} \\
b & \longmapsto \widehat{b}
\end{aligned}
$$

onde $\widehat{a}, \widehat{b}$ são como nos teoremas 3.3 .3 e 3.3 .5 respectivamente. Além disso a sobrejetividade de $I_{\Psi}(k) \hookrightarrow \pi_{1}\left(\widehat{N}, z^{*}\right) \longrightarrow \pi_{1}\left(N, z^{*}\right)$ estará garantida se demonstramos que $a, b \in I_{\Psi}(k)$, para algum $k \in\{1, \ldots, d\}$ onde $a, b$ são os geradores de $\pi_{1}\left(N, z^{*}\right)$. De fato:

1. No caso do toro, pelo teorema 3.3.3 $\widehat{a}$ fixa 1 , portanto $a \in I_{\Psi}(1)$ e $i_{*}(a)=a \in$ $\pi_{1}\left(\widehat{N}, z^{*}\right)$. O mesmo teorema garante que ou $b[a, b]$ ou $b\left[a^{2}, b\right]$ está em $I_{\Psi}(1)$, seja qual for o caso, temos $b \in \operatorname{Im}\left(i_{*}\right)$ pois $\pi_{1}\left(N, z^{*}\right)$ é abeliano e portanto qualquer comutador torna-se a identidade.

2. No caso da garrafa de Klein, segundo o teorema $3.3 .5 \widehat{a}$ fixa 1 assim como $\widehat{b}[\widehat{a}, \widehat{b}]_{-}^{q}$, para algum $q \in \mathbb{Z}^{+}$. Portanto $a, b[a, b]_{-}^{q} \in I_{\Psi}(1)$ e $i_{*}(a)=a, i_{*}\left(b[a, b]_{-}^{q}\right)=b \in$ $\pi_{1}\left(N, z^{*}\right)$, pois $[a, b]_{-}=1 \mathrm{em} \pi_{1}\left(\widehat{N}, z^{*}\right)$.

Dizemos que o subgrupo $H$ de $\pi_{1}(N)$ corresponde ao revestimento ramificado $\left(M, \phi, N, B_{\phi}\right)$ se $\phi_{*}\left(\pi_{1}(M)\right)=H$.

Teorema 5.4 Seja $N$ uma superficie fechada diferente da esfera e do plano projetivo, $d \in \mathbb{Z}^{+}, H \subset \pi_{1}\left(N, z^{*}\right)$ um subgrupo $€ \mathscr{D}=\left\{A_{1}, \ldots, A_{k}\right\}$ um dado de ramificação virtual. 
Então as seguintes afirmações são equivalentes:

1. O subgrupo $H$ corresponde a algum revestimento ramificado conexo entre superficies fechadas que realiza $\mathscr{D}$.

2. $H$ é um subgrupo de indice finito $l$ tal que $l \mid d$ e para cada $i \in\{1, \ldots, k\}$ existem $l$ partiçoes

$$
B_{i 1}=\left[d_{i 11}, \ldots, d_{i 1 r_{i 1}}\right], \ldots, B_{i l}=\left[d_{i l 1}, \ldots, d_{i l r_{i l}}\right]
$$

do número $d / l$ tais que

$$
A_{i}=B_{i 1} \sqcup \cdots \sqcup B_{i l}=\left[d_{i 11}, \ldots, d_{i 1 r_{i 1}}, \ldots, d_{i l 1}, \ldots, d_{i l r_{i l}}\right] .
$$

A versão algébrica do teorema anterior é:

Teorema 5.5 Seja $\pi=\left\langle a_{1}, \ldots, a_{n}, s_{1}, \ldots, s_{k} \mid \Pi^{*}\left(s_{1} \ldots s_{k}\right)=1\right\rangle$ a presentação de um. grupo, para $n \geq 2, k \geq 1, e \prod^{*}=\prod_{i=1}^{n / 2}\left[a_{2 i-1}, a_{2 i}\right](n=2 g)$, ou $\prod^{*}=a_{1}^{2} \ldots a_{n}^{2}$. Seja $H$ um subgrupo de $\pi /\left\langle\left\langle s_{1}, \ldots, s_{k}\right\rangle\right\rangle$ onde $\left\langle\left\langle s_{1}, \ldots, s_{k}\right\rangle\right\rangle$ é o menor subgrupo normal contendo os elementos $s_{1}, \ldots, s_{k}$. Sejam $\sigma_{1}, \ldots, \sigma_{k} \in S_{d}-\{i d\}$ permutaçoes tais que $\prod_{i=1}^{k} \sigma_{i} \dot{e}$ uma permutação par, $\epsilon$ denotemos por $A_{i}$ a coleção dos comprimentos dos ciclos de $\sigma_{i}$. As seguintes afirmaçoés são equivalentes:

1. Existe um homomorfismo $\Psi: \pi \longrightarrow S_{d}$ tal que :

(a) O subgrupo $\Psi(\pi)<S_{d}$ age transitivamente sobre $\{1, \ldots, d\}$.

(b) $\Psi\left(s_{i}\right)$ é conjugado de $\sigma_{i}$

(c) A imagem da composição $I_{\Psi}(1) \hookrightarrow \pi \longrightarrow \pi /\left\langle\left\langle s_{1}, \ldots, s_{k}\right\rangle\right\rangle$ é $H$.

2. Hé um subgrupo de indice finito l tal que $l \mid d$ e para cada $i \in\{1, \ldots, k\}$ existem $l$ partições

$$
B_{i 1}=\left[d_{i 11}, \ldots, d_{i 1 r_{i 1}}\right], \ldots, B_{i l}=\left[d_{i l 1}, \ldots, d_{i l r_{i l}}\right]
$$

do numero d/l tais que

$$
A_{i}=B_{i 1} \sqcup \cdots \cup B_{i l}=\left[d_{i 11}, \ldots, d_{i 1 r_{i 1}}, \ldots, d_{i l 1}, \ldots, d_{i r_{i l}}\right]
$$


Notemos que os grupos fundamentais $\pi_{1}\left(\widehat{N}, z^{*}\right)$ e $\pi_{1}\left(N, z^{*}\right)$ são isomorfos a os grupos $\pi \mathrm{e} \pi /\left\langle\left\langle s_{1}, \ldots, s_{k}\right\rangle\right\rangle$ respectivamente, e que estes isomorfismos respeitam as projeções $\pi_{1}\left(\widehat{N}, z^{*}\right) \longrightarrow \pi_{1}\left(N, z^{*}\right), \pi \longrightarrow \pi /\left\langle\left\langle s_{1}, \ldots, s_{k}\right\rangle\right\rangle$. Além disso, do corolário 5.3 segue que os teoremas 5.4 e 5.5 são equivalentes.

Demonstração do Teorema 5.5 no caso primitivo: Se $H=\pi /\left\langle\left\langle s_{1}, \ldots, s_{k}\right\rangle\right\rangle$, então $l=1$ e a segunda afirmação é sempre válida. Vamos ver que a primeira é sempre válida também.

Consideremos o caso $n=2, k=1, \pi=\left\langle a_{1}, a_{2}, s_{1} \mid\left[a_{1}, a_{2}\right] s_{1}=1\right\rangle$ e $A_{1}=\left[d_{1}, \ldots, d_{r}\right]$. Pelo corolário 3.3.4 existem permutaçōes $\widehat{a_{1}}, \widehat{a_{2}} \in S_{d}$ tais que seu comutador é o produto de ciclos de comprimentos $d_{1}, \ldots d_{r}$. Assim podemos definir a seguinte representação de Hurwitz:

$$
\begin{aligned}
\Psi: \pi & \longrightarrow S_{d} \\
a_{1} & \longmapsto \widehat{a_{2}} \\
a_{2} & \longmapsto \widehat{a_{1}} \\
s_{1} & \longmapsto\left[\widehat{a_{1}}, \widehat{a_{2}}\right]
\end{aligned}
$$

daqui as condições (a), (b) seguem das propriedades (1), (2) do corolário 3.3.4. Do mesmo corolário temos que o 1 fica fixo por $\widehat{a_{1}}$ e por $\widehat{a_{2}}\left[\widehat{a_{1}}, \widehat{a_{2}}\right]$ ou $\widehat{a_{2}}\left[{\widehat{a_{1}}}^{2}, \widehat{a_{2}}\right]$, donde $I_{\Psi}(1) \hookrightarrow \pi \longrightarrow$ $\pi /\left\langle\left\langle s_{1}\right\rangle\right\rangle$ é sobrejetora.

Seja agora $k \geq 2$ e $A_{i}=\left[d_{i_{1}}, \ldots, d_{i_{\gamma_{i}}}\right], i=1, \ldots, k$. Peguemos permutações $\widehat{s_{i}} \in S_{d}$ tais que seus ciclos tenham comprimentos $d_{i_{1}}, \ldots, d_{i_{r_{i}}}$ respectivamente. Se a permutação $\widehat{c}=\widehat{s_{1}} \ldots \widehat{s_{k}} \neq \widehat{e}$, onde $\widehat{e}$ é a permutação identidade, é só aplicar o corolário 3.3.4. Mas se $\widehat{s_{1}} \ldots \widehat{s_{k}}=\widehat{\epsilon}$ analisamos os seguintes casos:

(1) se em algum $\widehat{s}_{i}$ existe um ciclo de comprimento maior ou igual que 3.

(2) se todos os ciclos têm comprimento menor ou igual que 2 e $d \geq 3$.

(3) se $d=2$.

Se acontece (1), é só trocar $\widehat{s}_{i}$ por $\widehat{s}_{i}^{-1}$. Se (2), trocamos em um $\widehat{s_{i}} \neq \widehat{e}$ um símbolo de um ciclo de comprimento 2 por um símbolo de outro ciclo qualquer. Assim, sem mudaro comprimento dos ciclos, o novo $\widehat{s_{i}}$ é tal que o produto $\widehat{s_{1}} \ldots \widehat{s_{k}}$ é diferente da identidade, 
logo pode-se aplicar o corolário 3.3.4 e definimos a seguinte representação de Hurwitz

$$
\begin{aligned}
\Psi: \pi & \longrightarrow \widehat{S}_{d} \\
a_{1} & \longrightarrow \widehat{a_{2}} \\
a_{2} & \longmapsto \widehat{a_{1}} \\
s_{i} & \longmapsto \widehat{s_{i}}
\end{aligned}
$$

donde $\left[a_{1}, a_{2}\right]\left(s_{1} \ldots s_{k}\right) \longmapsto[\widehat{a}, \widehat{b}]^{-1}\left(\widehat{s_{1}} \ldots \widehat{s_{k}}\right)=\widehat{e}$ e como no caso $k=1$, o resto da primeira condição do teorema segue do mesmo corolário.

Se temos (3), existe um $\widehat{s_{i}} \neq \widehat{e}$. Peguemos permutações arbitrarias e diferentes $\widehat{a}, \widehat{b} \in$ $S_{2}$ e automaticamente a primeira condição é satisfeita.

Assumamos agora que $\pi=\left\langle a_{1}, \ldots, a_{2 g}, s_{1}, \ldots, s_{k} \mid \prod^{*}\left(s_{1} \ldots s_{k}\right)=1\right\rangle$ com $m \geq 1 \mathrm{e}$ $\Pi^{*}=\prod_{i=1}^{g}\left[a_{2 i-1}, a_{2 i}\right]$. Definamos a representação de Hurwitz enviando $a_{1}, a_{2}, s_{1}, \ldots, s_{k}$ como no caso analisado anteriormente e levemos $a_{3}, \ldots, a_{2 g}$ à permutação identidade.

Para o caso $\pi=\left\langle a_{1}, \ldots, a_{n}, s_{1}, \ldots, s_{k} \mid \prod^{*}\left(s_{1} \ldots s_{k}\right)=1\right\rangle$ com $m \geq 1, n \geq 2 \mathrm{e}$ $\Pi^{*}=a_{1}^{2} \ldots a_{n}^{2}$ procedemos analogamente só que aplicando o corolário 3.3.6.

Já que os teoremas 5.4 e 5.5 são equivalentes, temos demonstrado então o teorema 5.4 no caso primitivo, isto é quando $H=\pi_{1}\left(N, z^{*}\right)$.

Demonstração dos teoremas 5.4 e 5.5 no caso geral: Seja $\phi: M \longrightarrow N$ um revestimento ramificado tal que $\phi_{*}\left(\pi_{1}(M)\right)=H$. Consideremos o revestimento não ramificado $p: \widetilde{N} \longrightarrow N$ que corresponde ao subgrupo $H$. Então $\phi$ pode-se levantar a $\tilde{\phi}: M \longrightarrow \tilde{N}$ e o diagrama

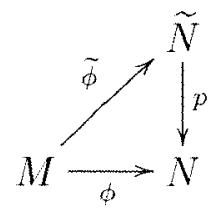

comuta. Agora, qualquer ponto $x_{i} \in B_{\phi}$ tem $l=\left[\pi_{1}(N): H\right]$ preimagens em $\tilde{N}$ e daqui, pela comutatividade do diagrama, a partição $A_{i}=\left[d_{i_{1}}, \ldots, d_{i_{r_{i}}}\right]$ pode-se escrever como a união de $l$ partições do inteiro $d / l$. 
Por outro lado, seja $p: \widetilde{N} \longrightarrow N$ o revestimento não ramificado que corresponde ao subgrupo $H$. Seja $\widetilde{\mathscr{D}}=\left\{B_{11}, \ldots, B_{1 l}, \ldots, B_{k 1}, \ldots, B_{k l}\right\}$ um dado de ramificação virtual. Já que $\chi(\tilde{N})=l \cdot \chi(N) \leq 0$, a superfície $\widetilde{N}$ é diferente da esfera e do plano projetivo. Segue do teorema 5.4 para o caso primitivo que existe um revestimento ramificado conexo $h: M \longrightarrow \widetilde{N}$ que realiza $\widetilde{\mathscr{D}}$. Assim $\left(M, p \circ h, N, p\left(B_{\tilde{\phi}}\right)\right)$ é o revestimento procurado. 


\section{Capítulo 6}

\section{Sobre Superfícies com bordo}

Neste capítulo resolvemos a Questão 1 e a Questão 2 quando $N$ é uma superfície com bordo e $d \in \mathbb{Z}^{+}$finito.

Seja $N$ uma superfície fechada. Escolhamos sobre ela um número $n$ finito de discos fechados disjuntos e tiremos o interior de cada um deles. A superfície $N^{n *}$ assim obtida é uma superfície com $n$ componentes de bordo. Reciprocamente, se $N^{n *}$ é uma superfície com $n$ componentes de bordo, cada componente é homeomorfa a um círculo. Portanto se pegamos $n$ discos fechados e fazemos a colagem do $i$-ésimo disco com a $i$-ésima componente, obtemos uma superfície fechada $N .^{1}$

Enunciemos novamente as questões que queremos resolver:

Questão 1: Dada uma superficie $N^{n *}$, um candidato d para grau e um conjunto de partiçoes $\mathscr{D}=\left\{A_{i}\right\}_{i \in I}$ de d onde cada termo de $A_{i}, i \in I$, é finito, será que existe um revestimento ramificado conexo $\left(M^{*}, \phi, N^{n *}, B_{\phi}\right)$ tal que realize $\mathscr{D} ?$

Questão 2: Seja $H \subset \pi_{1}\left(N^{n *}\right)$ um subgrupo de indicel finito, $d \in \mathbb{Z}^{+}, e \mathscr{T}=\left\{A_{1}, \ldots, A_{k}\right\}$ um conjunto de partições de $d$. Existe um revestimento ramificado conexo $\left(M^{*}, \phi, N^{n *}, B_{\phi}\right)$ de graud que realize $\mathscr{D}$ e tal que $\phi_{*}\left(\pi_{1}\left(M^{*}\right)\right)=H$ ?

\footnotetext{
${ }^{1}$ Ver [Ma], capítulo I, seção 10 , página 37 .
} 
Resposta à Questão 1: Observemos que se $N^{n *}$ é uma superfície com bordo, $d \in \mathbb{Z}^{+}$ e $\mathscr{D}=\left\{A_{1}, \ldots, A_{k}\right\}$ é um conjunto de partições de $d$, a existência de um revestimento ramificado que realize $\mathscr{D}$ depende da possibilidade de definir uma representação de Hurwitz, ${ }^{2}$ isto é, um homomorfismo $\Psi: \pi_{1}\left(\widehat{N}^{n *}\right) \longmapsto S_{d}$, onde $\widehat{N}^{n *}$ denota a superfície $N^{n *}$ depois de tirados $k$ pontos, $\left\{x_{1}, \ldots, x_{k}\right\}$. Mas tanto $\pi_{1}\left(N^{n *}\right)$ quanto $\pi_{1}\left(\widehat{N}^{n *}\right)$ são grupos livres, $\operatorname{com} \pi_{1}\left(N^{n *}\right) \subset \pi_{1}\left(\widehat{N}^{n *}\right)$, já que por cada $x_{i}$ tirado acrescenta-se em um o número de geradores de $\pi_{1}\left(N^{n *}\right)$. Logo, temos absoluta liberdade para definir a representação, pois não precisaremos cuidar de relação nenhuma. Assim, se $\alpha_{i} \in \pi_{1}\left(\widehat{N}^{n *}\right)$ é a classe obtida a partir de $x_{i}$, definimos $\alpha_{i} \longmapsto \widehat{\alpha_{i}} \in S_{d}$ tal que a coleção dos comprimentos dos ciclos de $\widehat{\alpha_{i}}$ determina a partição $A_{i}$. E para garantir a conexidade do revestimento basta só definir $\Psi\left(a_{j}\right)$ como um $d$-ciclo, sendo $a_{j}$ um gerador de $\pi_{1}\left(N^{n *}\right)$. Portanto precisamos $\pi_{1}\left(N^{n *}\right)$ não trivial, isto é, se $N=\mathbb{S}^{2}$ então $n>1$.

Resposta à Questão 2 no caso primitivo: Seja $H=\pi_{1}\left(N^{n *}\right)$. Se $N^{n *}$ é obtida a partir da esfera e $n=2$, então $\pi_{1}\left(N^{n *}\right)=F(a)$ é um grupo livre em um gerador. Para $k=1$ tem-se $\mathscr{D}=\{A\} \operatorname{com} A=\left[d_{1}, \ldots, d_{r}\right]$. Assim, $\pi_{1}\left(\widehat{N}^{n *}, z^{*}\right)=F(a, \alpha)$ é um grupo livre em dois geradores. Posso livremente definir a seguinte representação:

$$
\begin{aligned}
\Psi: F(a, \alpha) & \longrightarrow S_{d} \\
a & \longmapsto\left(c_{1} c_{2} \ldots c_{d}\right)=\widehat{a} \\
\alpha & \longmapsto\left(z_{1}\right)\left(z_{2}\right) \ldots\left(z_{r}\right)=\widehat{\alpha}
\end{aligned}
$$

onde o $d$-ciclo $\widehat{a}$ garante a conexidade e $\left(z_{i}\right)$ é um $d_{i}$-ciclo para $i=1, \ldots, r$.

É o revestimento ramificado $\left(M^{*}, \phi, N^{n *}, B_{\phi}\right)$, associado a $\Psi$, primitivo? ou equivalentemente, é $\phi_{*}\left(\pi_{1}\left(M^{*}\right)\right)=F(a)$ ?

Observemos que o teorema 5.1 é válido para este tipo de superfícies, assim, pelo corolário 5.2, para responder esta pergunta, basta mostrar que existe $\beta \in I_{\Psi}(1)$ tal que

\footnotetext{
${ }^{2}$ Ver corolário 2.2 .
} 
$i_{*}(\beta)=a$, onde $i_{*}$ é o homomorfismo induzido pela inclusão $i: \widehat{N}^{n *} \hookrightarrow N^{n *}$.

Sabemos que deve existir $j \in\{1, \ldots, r\}$ tal que $1 \in\left(z_{j}\right)$.

1. Se $d_{j}>1$, definimos $\widehat{a}$ tal que:

$$
\begin{aligned}
& c_{1}=1 . \hat{\alpha} \\
& c_{2}=1
\end{aligned}
$$

pois $\widehat{\alpha} \widehat{a}$ vai fixar o 1 :

$$
\text { 1. } \widehat{\alpha} \widehat{a}=c_{1} \cdot \widehat{a}=c_{2}=1 \text {. }
$$

Além disso $i_{*}(\alpha a)=a$, portanto é só fazer $\beta=\alpha a$.

2. Se $d_{j}=1$. Notemos que $a^{d} \in \phi_{*}\left(\pi_{1}(M)\right)$ já que $\widehat{a}$ é um $d$-ciclo, basta então mostrar que existe $\gamma \in I_{\Psi}(1)$ tal que $i_{*}(\gamma)=a^{h}$, para $h \in \mathbb{Z}^{+},(h, d)=1$. Pois pelo lema de Bezout existem inteiros $p, q$ tais que $p h+q d=1$, e teremos que $a=a^{p h} a^{q d} \in$ $\phi_{*}\left(\pi_{1}(M)\right)$.

Sabemos que tem que existir um $d_{i}>1, i \neq j$, pois $\widehat{\alpha}$ é diferente da identidade. Seja $c_{t} \in\left(z_{i}\right)$, e definamos $\widehat{a}$ tal que:

$$
\begin{aligned}
& c_{t-1}=1 \\
& c_{t+1}=c_{t} \cdot \widehat{\alpha}
\end{aligned}
$$

$\operatorname{assim} \widehat{a} \widehat{\alpha} \widehat{a}^{d-2}$ fixa o 1 :

$$
\text { 1. } \widehat{a} \widehat{\alpha} \widehat{a}^{d-2}=c_{t} \cdot \widehat{\alpha a} \widehat{a}^{d-2}=c_{t+1} \cdot \widehat{a}^{d-2}=c_{t-1}=1
$$

e mais do que isso, $i_{*}\left(a \alpha a^{d-2}\right)=a^{d-1}$. Portanto é só definir $\gamma=a \alpha a^{d-2}$.

O caso $k>2$ reduz-se ao caso anterior, pois já que $F\left(a, \alpha_{1}, \ldots, \alpha_{k}\right)$ é livre, basta definir o $d$-ciclo $\widehat{a}$ dependendo de $\widehat{\alpha_{1}}$ para garantir a primitividade. Analogamente para $n>2$, pois sendo $\pi_{1}\left(N^{n *}\right)=F\left(a_{1}, \ldots, a_{n-1}\right)$ um grupo livre em $n-1$ geradores, é só definir o homomorfismo $\Psi: F\left(a_{1}, \ldots, a_{n-1}, \alpha_{1}, \ldots, \alpha_{k}\right) \longrightarrow S_{d}$ tal que leve $\alpha_{i} \longmapsto \sigma_{i}, a_{1} \longmapsto \widehat{a_{1}}=$ $\left(c_{1} \ldots c_{d}\right)$ e $a_{j} \longmapsto 1,2 \leq j \leq n-1$, onde o comprimento dos ciclos de $\sigma_{i}$ obedece à partição $A_{i}$ e o $d$-ciclo $\widehat{a}_{1}$ define-se dependendo de $\widehat{\alpha_{1}}$ para garantir a primitividade. 
Se $N^{n *}$ é obtida a partir do toro, a resposta reduz-se ao caso anterior, pois $\pi_{1}\left(\mathbb{T}^{n *}\right) \cong$ $\pi_{1}\left(\mathbb{S}^{(n+2) *}\right)$ já que são grupos livres sobre conjuntos da mesma cardinalidade, onde $\mathbb{S}$ está representando a esfera. Aliás, pela mesma razão, $\pi_{1}\left(\mathbb{T}_{g}^{n *}\right) \cong \pi_{1}\left(\mathbb{S}^{(2 g+n) *}\right)$, onde $\mathbb{T}_{g}$ é a soma conexa de $g$ toros. Analogamente acontece se $N^{n *}$ é obtida a partir do plano projetivo pois $\pi_{1}\left(\mathbb{P}^{n *}\right) \cong \pi_{1}\left(\mathbb{S}^{(n+1) *}\right)$ e em geral $\pi_{1}\left(\mathbb{P}_{g}^{n *}\right) \cong \pi_{1}\left(\mathbb{S}^{(g+n) *}\right)$, onde $\mathbb{P}_{g}$ é a soma conexa de $g$ planos projetivos.

Resposta à Questão 2 no caso geral: Segue de copiar o teorema 5.4 junto com a sua demonstração mas usando o caso primitivo que acabamos de resolver . 


\section{Referências Bibliográficas}

[AS] Ahlfors, L.; Sario, L.: Riemann Surfaces. Princeton (1960).

[BGKZ1] Bogatiy, S.; Gonçalves, D.L.; Kudryavtseva, E,; Zieschang, H.: Realization of Primitive Branched Coverings over Closed Surfaces. To appear in Kluwer Academic Publishers.

[BGKZ2] Bogatiy, S.; Gonçalves, D.L.; Kudryavtzeva, E.; Zieschang, H.: Construction of Branched Coverings over Closed Surfaces following the Hurwitz Approach. Central Europ. J. of Math. 2, 184-197 (2003).

[DM] Dixon, J.D.; Mortimer, B.: Permutation Groups. Springer-Verlag (1996).

[EKS] Edmonds, A.L.; Kulkarni, R.S.; Stong, R.E.: Realizability of Branched Coverings of Surfaces. Trans. Amer. Math. Soc. 282, 773-790 (1984).

[Ez] Ezell, C.L.: Branch Point Structure of Covering Maps onto Nonorientable Surfaces. Trans. Amer. Math. Soc. 243, 123-133 (1978).

[Fo] Forster, O.: Lectures on Riemann Surfaces. Springer - Veralg (1981).

[Fu] Fulton, W.: Algebraic Topology. A First Course. Springer-Verlag (1995).

[Hu] Husemoller, D.H.: Ramified Coverings of Riemann Surfaces. Duke Math. J. 29, $167-174(1962)$.

[Lee] Lee, J.M.: Introduction to Topological Manifolds. Springer-Verlag (2000). 
[Ma] Massey, W.S.: Algebraic Topology: An introduction. Harcoyrt, Brace \& World (1967).

[Ro] Rotman, J.J.: The Theory of Groups. An introduction. Allyn and Bacon, Inv. (1965).

[St] Stö̈low, S.: Leçons sur les Principies Topologiques de la Théorie des Fonctions Analytiques. Gauthier-Villars (1956).

[Wi] Wielandt, H.: Finite Permutation Groups. Academic Press Inc. (1964). 


\section{Índice Remissivo}

conjunto

contínuo, 2

realizável, 11

defeito

de um ponto, 10

do revestimento, 10

domínio

aberto, 2

fechado, 3

Jordan, 6

máximo, 2

normal, 3

função primitiva, 51

Hurwitz

condições de, 39

fórmula de Riemann-, 15

representação, 13

teorema de, 13

ramificação

indice de, 1

dado de, 10

virtual, 37

ponto, 1 revestimento

não ramificado, 10

associado, 9

orientável duplo, 16

ramificado, 9

conexo, 10

não orientável, 40

orientável, 40

primitivo, 51

superfície de, 9

superfícies fechadas, 14

transformação interior, 2 